\title{
Scale-free Fluctuations in Bose-Einstein Condensates, Quantum Dots and Music Rhythms
}

\section{Dissertation}

\author{
zur Erlangung des Doktorgrades
}

der Mathematisch-Naturwissenschaftlichen Fakultäten der Georg-August-Universität zu Göttingen

vorgelegt von

\section{Holger Hennig}

\author{
aus Hamburg
}

Göttingen 2009 
D7

Referent

: Prof. Dr. Theo Geisel

Korreferent

: Prof. Dr. Kurt Schönhammer

Tag der mündlichen Prüfung : 27.05.2009 


\begin{abstract}
Mesoscopic systems are prone to substantial fluctuations that typically can not be neglected or avoided. The understanding of the origin and the consequences of these fluctuations (e.g. for transport measurements) is thus a fundamental part of the theory of mesoscopic systems. We will encounter scale-free fluctuations in different kinds of complex nonlinear systems in this thesis, which consists of two main parts. The first part deals with Bose-Einstein condensates (BECs) in leaking optical lattices. Experimentalists have achieved an extraordinary level of control over BECs in optical traps in the past decade, which allows for the investigation of complex solid state phenomena and the emerging field of 'atomtronics' promises a new generation of nanoscale devices. It is therefore both of fundamental and technological importance to understand the dynamics and transport properties of BECs in optical lattices. We study the outgoing atomic flux of BECs loaded in a one dimensional optical lattice with leaking edges, using a mean field description provided by the discrete nonlinear Schrödinger equation with nonlinearity $\Lambda$. We find that for a nonlinearity larger than a threshold $\Lambda>\Lambda_{b}$ the dynamics evolves into a population of discrete breathers, preventing the atoms from reaching the leaking boundaries. We show that collisions of other lattice excitations with the outermost discrete breathers result in avalanches, i.e. jumps of size $J$ in the outgoing atomic flux, which follow a scale-free distribution $\mathcal{P}(J) \sim 1 / J^{\alpha}$ characterizing systems at a phase transition. Our results are also relevant in a variety of other contexts, e.g. coupled nonlinear optical waveguides.

In the second part, fractal fluctuations in two different complex systems are studied. Firstly, conductance fluctuations in mesoscopic systems (such as quantum dots) are considered, which are a sensitive probe of electron dynamics and chaotic phenomena. Using the standard map as a paradigmatic model, we show that classical transport through chaotic Hamiltonian systems in general produces fractal conductance curves. This might explain unexpected results of experiments in semiconductor quantum dots where a dependence of the fractal dimension on the coherence length was observed. Furthermore, we predict fractal fluctuations in the conductance of low-dimensional Hamiltonian systems with purely chaotic phase space.

Secondly, we investigate temporal (fractal) fluctuations of human music rhythms compared with an exact pattern, e.g. given by a metronome. We show that the temporal fluctuations in simple as well as in more complex music rhythms are generic in the sense, that Gaussian $1 / f^{\beta}$ noise is produced, no matter whether the rhythmic task is accomplished with hands, feet, the voice or a combination of these. Professional audio editing software includes a so-called 'humanizing' feature, which adds deviations $\xi_{n}$ to a given audio sequence, where $\xi_{n}$ is white noise. We demonstrate that $1 / f$ humanized music that we created is rated significantly better by listeners than conventionally humanized sequences.
\end{abstract}




\section{Kurzfassung}

Mesoskopische Systeme unterliegen substanziellen Fluktuationen, die typischerweise nicht vernachlässigt oder vermieden werden können. Das Verständnis des Ursprungs und der Folgen dieser Fluktuationen (z.B. für Transportmessungen) ist daher ein fundamentaler Teil der Theorie mesoskopischer Systeme. In dieser Arbeit, welche aus zwei Teilen besteht, werden uns skalenfreie Fluktuationen in verschiedenen komplexen nichtlinearen Systemen begegnen. Der erste Teil handelt von Bose-Einstein Kondensaten (BECs) in undichten optischen Gittern. Experimentatoren haben in der letzten Dekade einen außerordentlichen Grad an Kontrolle über BECs in optischen Fallen erreicht, was die Untersuchung von komplexen Festkörperphänomenen ermöglicht und das aufkommende Feld 'Atomtronics' verspricht eine neue Generation von Nanobausteinen. Es ist daher sowohl von fundamentaler als auch von technologischer Bedeutung die Dynamik und die Transporteigenschaften von BECs in optischen Gittern zu verstehen. Wir untersuchen den Fluss von Atomen eines BECs aus einem eindimensionalen optischen Gitter mit undichtem Rand und benutzen eine Molekularfeld-Näherung gegeben durch die diskrete nichtlineare Schrödingergleichung mit Nichtlinearität $\Lambda$. Wir beobachten, dass bei einer Nichtlinearität größer als ein Schwellenwert $\Lambda>\Lambda_{b}$ die Dynamik zur Entstehung von diskreten Solitonen führt, welche die Atome davon abhalten, den undichten Rand zu erreichen. Wir zeigen, dass Kollisionen von anderen Gitteranregungen mit den äußersten diskreten Solitonen zu Lawinen führen, d.h. Sprünge der Größe $J$ in dem Fluss von Atomen, die einer skalenfreien Verteilung $\mathcal{P}(J) \sim 1 / J^{\alpha}$ folgen, was Systeme an einem Phasenübergang charakterisiert. Unsere Ergebnisse sind auch relevant in diversen anderen Kontexten, z.B. gekoppelte nichtlineare optische Wellenleiter.

Im zweiten Teil befassen wir uns mit fraktalen Fluktuationen in zwei verschiedenen komplexen Systemen. Zunächst werden Leitwertfluktuationen in mesoskopischen Systemen (wie zum Beispiel Quantenpunkte) betrachtet, die eine sensible Sonde für die Dynamik von Elektronen und chaotische Phänomene sind. Mittels der Standardabbildung als paradigmatisches Modell der Dynamik im gemischten Phasenraum wird gezeigt, dass der klassische Transport durch Hamiltonsche Systeme ganz allgemein fraktale Leitwertkurven hervorbringt. Dies könnte unerwartete Ergebnisse von Experimenten mit Halbleiter-Quantenpunkten erklären, bei denen eine Abhängigkeit der fraktalen Dimension von der Kohärenzlänge beobachtet wurde. Darüber hinaus sagen wir fraktale Fluktuationen in dem Leitwert niedrigdimensionaler Hamiltonscher Systeme mit rein chaotischem Phasenraum vorher.

Zweitens betrachten wir zeitliche (fraktale) Fluktuationen von menschlichen Musikrhythmen verglichen mit einem exakten Muster, z.B. gegeben durch ein Metronom. Es wird gezeigt, dass zeitliche Fluktuationen in einfachen und in komplexeren Musikrhythmen generisch sind, in dem Sinne, dass Gaußsches $1 / f^{\beta}$ Rauschen produziert wird, ganz gleich ob eine rhythmische Aufgabe mit Händen, Füßen, der Stimme oder einer Kombination dieser ausgeführt wird. Professionelle AudioBearbeitungssoftware beinhaltet ein sogenanntes 'Humanizing'-Werkzeug, welches Abweichungen $\xi_{n}$ zu einer gegebenen Audiosequenz hinzufügt, wobei $\xi_{n}$ weisses Rauschen ist. Wir zeigen, dass von uns kreierte $1 / f$-humanisierte Musik signifikant besser von Zuhörern bewertet wird als konventionell humanisierte Sequenzen. 


\section{Acknowledgments}

First, it is a pleasure for me to thank my advisor, Theo Geisel, for the possibility to work in his unique group, for illuminating discussions, and for constant support of my plans. With his continuous effort he creates excellent working conditions and a great atmosphere in the institute.

Special thanks are due to Ragnar Fleischmann, for supporting me in so many ways throughout the years, for teaching me nonlinear dynamics, inspiring discussions, for giving me the freedom to pursue a variety of different projects and own ideas, and for his kind-heartedness and humor.

I would like to thank Tsampikos Kottos for the fruitful collaboration on BECs, I learned a lot during our active video conferences that included plenty of questions and ideas. Also, it was a pleasure to collaborate with Gim Seng Ng, thank you as well for being a delightful office mate during your visits in Göttingen in summers.

I had the opportunity to stay at Boston University from May-September 2008 and to learn from and work with David Campbell and Jérôme Dorignac. I would like to thank David for his great hospitality, for valuable and inspiring discussions and for making that wonderful research visit possible. His deep knowledge of many fields has been an invaluable resource. Special thanks to Jérôme for a fruitful work together that taught me a lot, for illusive discussions on the trimer and about "Gott und die Welt", for his friendship, and for coffee at espresso royal. I would also like to thank Rafael Hipolito for valuable discussions on BECs.

Concerning the project on music rhythms, first, I would like to thank Fabian Theis, Annette Witt and Jan Nagler for the fruitful collaboration and for stimulating discussions. I wish to gratefully acknowledge the interdisciplinary collaboration with the psychology department, in particular York Hagmayer, Anneke Fredebohm (who investigated humanized music in her diploma thesis) and Christine Paulus. For the creation of the humanized song, the wonderful team at Cubeaudio Recording Studio deserves great thanks, also for providing audio data. Special thanks to Götz for a highly creative collaboration - and for breakfast. I am also indebted to the people at Max-Planck-Innovation (in particular Bernd Ctortecka) and Alexander Bach for filing the patent and their enthusiasm and to the Göttingen Symphonic Orchestra (esp. Thomas Scholz).

I would like to thank everyone in the Geisel group for the great and lively atmosphere, from which I benefited a lot both scientifically and socially. Special thanks to Fabio and Jansky who have become friends. I would like to thank Marc Timme for helpful discussions and his amity and my former and current office mates Antonio Méndez-Bermúdez, Raoul Martin Memmesheimer, Sven Jahnke and Rob Shaw for the friendly atmosphere and for their patience while I am writing up, and the transport group - Oliver Bendix, Jakob Metzger and Kai Bröking for help and 
useful discussions. For support concerning computer-related questions, I am indebted to Denny Fliegner and Yorck-Fabian Beensen. I would like to thank Katja Fiedler and Lishma Anand as part of the midday "mtm" group and my gardener buddy with the "green thumb" Carsten Grabow. Thanks to Frank van Bussel for proofreading. I also want to thank the secretaries and the administrative staff. I wish to acknowledge the head of the institutes management, Kerstin Mölter, for her support and enthusiasm. I wish to thank everyone who helped as a test person.

I would like to thank my friends for being who they are. I wish to thank my parents and my brother for always being there for me. Finally, to Sabine, thank you for your support, your smile and your patience. 
To my parents 


\section{Contents}

\begin{tabular}{lll}
\hline 1 & Introduction & 10
\end{tabular}

\begin{tabular}{lll}
\hline 2 & Fundamentals & $\mathbf{1 3}$
\end{tabular}

2.1 Mesoscopic Systems and Fluctuations . . . . . . . . . . . . . . . . . 13

2.2 Transport and Diffusion in Phase Space . . . . . . . . . . . . . . . 14

$2.2 .1 \quad$ Standard Map . . . . . . . . . . . . . . . . . . 15

2.3 Fractal Analysis . . . . . . . . . . . . . . . . . . . . . . . . 17

2.3.1 Comparison of Different Methods . . . . . . . . . . . . . . . 18

2.4 Discrete Breathers $\ldots \ldots \ldots \ldots$. . . . . . . . . . . . . . 20

3 Avalanches of BECs in Optical Lattices 24

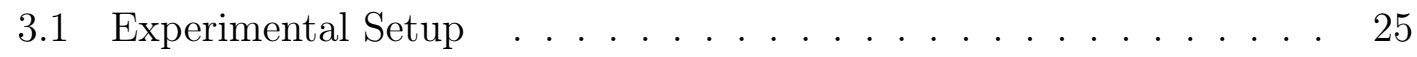

3.2 Discrete Nonlinear Schrödinger Equation . . . . . . . . . . . . . . . 27

$3.2 .1 \quad$ Estimating the Leakage Term . . . . . . . . . . . . . . . . . 28

3.3 Survival Probability: Avalanches . . . . . . . . . . . . . . . . . . . 29

3.4 Dynamics of BECs $\ldots \ldots \ldots$. . . . . . . . . . . . . . . . . . . . 30

3.5 Order Parameter $\ldots \ldots \ldots 33$

3.5 .1 Nature of the Phase Transition . . . . . . . . . . . . . . . 34

3.6 An Avalanche Event $\ldots \ldots \ldots$. . . . . . . . . . . . . . . . . . . . . . . 35

3.7 Origin of the Scale-free Avalanches . . . . . . . . . . . . . . . . 36

3.7 .1 Poincaré Section of the Trimer . . . . . . . . . . . . . . . . . . . . . . . . 36

3.7 .2 A Simple Hierarchical Model . . . . . . . . . . . . . . . . . . 39

3.7 .3 Test with Standard Map . . . . . . . . . . . . . . . . . . . . . . . . . . . . . . 40

3.8 Nonlinear Trimer . . . . . . . . . . . . . . . . . . . . . . . . . . . . . . 42

3.8.1 Equations of Motion . . . . . . . . . . . . . . . . . . . . . . . . . . . . . . . . . . 43

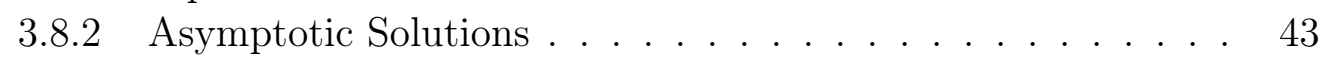

3.8.3 Peierls-Nabarro Energy Landscape . . . . . . . . . . . . . . . . . . . . 45

3.9 Possible Applications $\quad \ldots \ldots \ldots$. . . . . . . . . . . . . . . . . . . . . . 48

3.10 Conclusions . . . . . . . . . . . . . . . . . . . . . . . . . . . . . . . 49

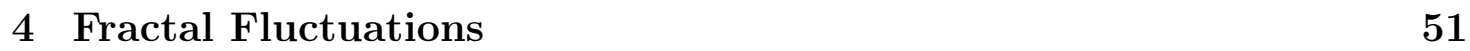

4.1 Mesoscopic Conductance Fluctuations . . . . . . . . . . . . . . . . 51

$4.1 .1 \quad$ Electron Billiards $\ldots \ldots \ldots \ldots$. . . . . . . . . . . . . . . 52

4.1 .2 Universal Conductance Fluctuations . . . . . . . . . . . 53

$4.1 .3 \quad$ Fractal Fluctuations: Semiclassics . . . . . . . . . . . . 54

$4.1 .4 \quad$ Experiments Beyond Semiclassics . . . . . . . . . . . . . . . . 56

$4.1 .5 \quad$ Fractal Fluctuations: Classical Origin . . . . . . . . . . . . . 57 


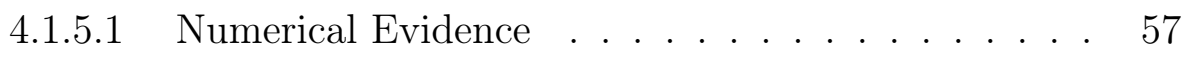

4.1.5.2 Model: Open Standard Map . . . . . . . . . . . . . 58

4.1.5.3 Analytics: Fractal Dimension . . . . . . . . . . . 60

4.1.5.4 Origin of the Power Law . . . . . . . . . . . . . . . 65

4.1 .6 Conclusions $\ldots \ldots \ldots \ldots$

4.2 Long-range Correlations in Music Rhythms . . . . . . . . . . . . . 67

4.2 .1 Error Time Series . . . . . . . . . . . . . . . . . . . 68

4.2 .2 Data Set \& Analysis . . . . . . . . . . . . . . . 70

$4.2 .3 \quad$ Modeling Rhythmic Drumming . . . . . . . . . . . . . . . . 76

4.2 .4 Humanizing Music Sequences . . . . . . . . . . . . . . . . . . 78

4.2 .4 .1 Drum Rhythms . . . . . . . . . . . . . . . . . . . 79

$4.2 .4 .2 \quad$ Creating Humanized Music . . . . . . . . . . . . . 81

$4.2 .4 .3 \quad$ Perception of Humanized Music . . . . . . . . . . . 82

4.2 .4 .4 Patent . . . . . . . . . . . . . . . . . 83

4.2 .5 Conclusions . . . . . . . . . . . . . . . . . . . . . . . . 84

$\begin{array}{llr}5 \text { Conclusions and Outlook } & 86\end{array}$

\begin{tabular}{lr}
\hline Appendix & 89
\end{tabular}

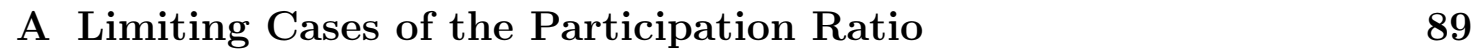

\begin{tabular}{|lr|}
\hline B Distribution of Islands (Mixed Phase Space) & 91
\end{tabular}

\begin{tabular}{lr|}
\hline C & Questionnaire and Lyrics \\
\hline
\end{tabular}

\begin{tabular}{ll|}
\hline D Audio Examples & 97
\end{tabular} 


\section{Chapter 1}

\section{Introduction}

The scientific and technological advances of the last decades have lead to the fabrication of two different kinds of mesoscopic systems. On the one hand, in a scale-down approach, electrical and optical devices are shrunk to a degree where intrinsic length scales of the material, such as the mean free path or the coherence length, become comparable with the system size. Thus the actual shape of the conductor or the individual positions of impurities gain important roles and the encounter of classical nonlinear dynamics and interference effects lead to complex quantum dynamics. On the other hand in a scale-up approach microscopic units are assembled to form larger and more complex entities as in the growing field of molecular electronics, allowing to technologically use the phenomena of complex quantum dynamics.

A special type of this scaled up systems are Bose-Einstein condensates in optical traps and lattices, as they combine the acuteness of atomic systems with the flexibility and formability of solid state systems opening the new field of "atomtronics".

All those mesoscopic systems have in common that either by their fabrication process or/and by their envisioned future function in some kind of circuitry, they are fundamentally coupled to the environment, so they have to be considered as open systems. This led to a recent enhanced interest in the theory of open (quantum) systems and complex scattering.

Transport through these open systems is due to their mesoscopic nature prone to substantial fluctuations that can not be neglected or avoided and whose understanding is thus a fundamental part of the theory of complex systems. In this work, we will encounter scale-free fluctuations in different kinds of complex nonlinear systems.

In Chap. 3 we will see how nonlinear localization leads to scale-free fluctuations in BECs in optical lattices in the framework of the discrete nonlinear Schrödinger equation (DNLS). We point out that although our focus is given to atomic BECs, our results are also relevant in a large variety of contexts (whenever the DNLS is adequate), most prominently in the light conduction in coupled nonlinear optical waveguides [1 5].

Experimentalists have achieved an extraordinary level of control over BECs in optical traps in the past decade, which allows for the investigation of complex solid state phenomena [6-13] and the emerging field of atomtronics promises a new generation of nanoscale devices. It is therefore both of fundamental and technological 
importance to understand the dynamics and transport properties of BECs in optical lattices. Here, we will show that if the optical lattice is opened at the ends, the statistics of the outgoing flux provides valuable and crucial information about the internal dynamics of the system.

We will study the decay of an atomic BEC population $N(\tau)$ from the leaking boundaries of an optical lattice using a mean field description provided by the DNLS. The DNLS, which will be described in detail in Sec. 3.2, is a lattice equation that contains a nonlinear term with prefactor $\Lambda$.

An exciting feature appearing in the framework of nonlinear lattices is the existence of spatially localized, time-periodic and stable (or at least long-lived) excitations, termed discrete breathers (DBs), which emerge due to the nonlinearity and discreteness of the system. In the DNLS with boundary dissipation, we will see that the internal systems dynamics evolves into generic initial conditions of DB states for a nonlinearity larger than a threshold $\Lambda>\Lambda_{b}$, preventing the atoms from reaching the leaking boundaries.

We show that collisions of other lattice excitations (e.g. "moving breathers", see Sec. 2.4 with the outermost DBs result in bursts of the outflux of sizes $\delta N$, i.e. steps in $N(\tau)$, which we call avalanches as for a whole range of $\Lambda$-values they follow a scale-free distribution, characterizing systems at a phase transition. We will see how the scale-free behavior reflects the complexity and the hierarchical structure of the underlying classical mixed phase space by reducing the system to few degrees of freedom yielding the closed nonlinear trimer.

Furthermore, in this framework, we will investigate the collision process of a stationary DB with a lattice excitation both analytically and numerically, which is work that was started during a research visit at Boston University from MaySeptember 2008.

While in Chap. 3 the transport properties of bosons in leaking optical lattices are described, in the first section of Chap. 4 we will consider fermions and discuss the electronic transport in open solid state mesoscopic systems. In these quantum systems (such as quantum dots, nanowires etc.) fluctuations of the conductance, are a sensitive probe of electron dynamics and chaotic phenomena. A prominent feature of electronic transport in mesoscopic systems is that the conductance as a function of an external parameter, e.g. a gate voltage or a magnetic field, shows reproducible fluctuations caused by quantum interference [14-16].

A prediction from semiclassical theory that inspired a number of both theoretical and experimental works in the fields of mesoscopic systems and quantum chaos was that in chaotic systems with a mixed phase space these fluctuations would result in fractal conductance curves [17, 18], i.e. when zooming into smaller and smaller scales of changes of e.g. the magnetic field, the conductance curve remains "rough" in a self-affine way. Such fractal conductance fluctuations (FCF) have since been confirmed in gold nanowires and in mesoscopic semiconductor quantum dots in various experiments [19 23]. In addition, FCF have more recently been predicted to occur in strongly dynamically localized [24] and in diffusive systems [25].

We will explain, that the conductance of purely classical (i.e. incoherent) lowdimensional Hamiltonian systems very fundamentally exhibits fractal fluctuations, as long as transport is at least partially conducted by chaotic dynamics and that 
the fractal dimension is governed by fundamental properties of chaotic dynamics. Thus mixed phase space systems and fully chaotic systems alike generally show fractal conductance fluctuations. This might explain the unexpected dependence of the fractal dimension of the conductance curves on the (quantum) phase breaking length observed in experiments on semiconductor quantum dots.

A completely different system where fractal fluctuations are found are music rhythms played by humans (Sec. 4.2). While in the case of FCF, we are interested in the structures on finer and finer scales, here the long-time correlations lead to the fractal nature of the fluctuations. Still, the idea of investigating human rhythms emerged from studying FCF.

Music performed by humans will always exhibit a certain amount of fluctuations compared with e.g. the steady beat of a metronome. It has been shown in the 1970s that loudness and pitch fluctuations in music exhibit $1 / f^{\beta}$ noise. Compositions in which the frequency and duration of each note were determined by $1 / f^{\beta}$ noise sources sounded much more pleasing to listeners than those comprising white noise sources [26, 27].

We will show that the temporal fluctuations in simple as well as in more complex music rhythms are generic in the sense, that Gaussian $1 / f^{\beta}$ noise is produced, no matter if the rhythmic task is accomplished with hands, feet, a combination of these or the voice.

Moreover, we will be led to an application by asking the question: Does the rhythmic structure of a piece of music sound better, when it is as exact as possible or are long-range correlations more favorable? Professional contemporary audio editing software include a so called 'humanizing' feature, which adds deviations $\xi_{n}$ to a given audio sequence, where $\xi_{n}$ is white noise. Hence, there exists a desire to let machine generated or modified music sound more natural. We created music that was humanized either with Gaussian $1 / f^{\beta}$ noise or white noise. To further investigate the perception of natural deviations in human music rhythms with more complex and realistic music pieces, an interdisciplinary diploma thesis in Psychology was initiated (Sec. 4.2.4.3).

The outline of the main part of the thesis is the following. In Chap. 2, some fundamental aspects of mesoscopic systems will be briefly reviewed. In Chap. 3 . we will analyze Bose-Einstein condensates in leaking optical lattices described by the DNLS yielding avalanches of ultracold atoms [28]. We will see that collisions of DBs with other lattice excitations lead to the observed avalanches. The collision process will be investigated analytically in the nonlinear trimer [29. In the next chapter, in Sec. 4.1, we consider fractal conductance fluctuations of classical origin in mesoscopic systems [30]. Finally, in Sec. 4.2 we are dealing with generic longrange correlations in human rhythmic drumming [31, 32]. 


\section{Chapter 2}

\section{Fundamentals}

\subsection{Mesoscopic Systems and Fluctuations}

In this section we will introduce the notion of mesoscopic systems, see e.g. [33] for a detailed review. Much of solid state theory and statistical physics is concerned with the properties of macroscopic systems. These are often considered while using the thermodynamic limit, i.e. the systems volume and particle number tend to infinity while their fraction remains constant. It is a convenient mathematical tool for obtaining bulk properties. Typically, the system approaches the macroscopic limit once its size is much larger than relevant characteristic length scales, which are

- the coherence length, which is the distance a particle travels before its initial phase is destroyed,

- the de Broglie wavelength, which is related to the kinetic energy of the particle,

- the mean free path, which is the distance that a particle travels before the initial momentum is destroyed.

On the other hand, in the microscopic limit, we encounter systems such as single atoms, where the laws of quantum mechanics govern the dynamics. Microscopic systems are identical systems and the properties are exactly reproducible (e.g. the transitions between energetic states in a hydrogen atom).

A mesoscopic system is a system in the intermediate size range between mi-

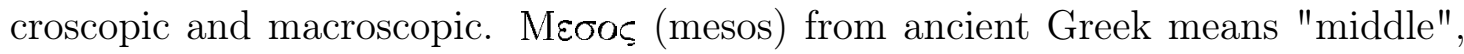
the word mesoscopic was coined by Van Kampen in 1981. The size range of a mesoscopic system depends on the relevant characteristic length scales (correlation length, wavelength and mean free path), which vary widely from one material to another and are also strongly affected by temperature, magnetic field etc. For this reason, mesoscopic transport phenomena have been observed in conductors having a wide range of dimensions from a few nanometers to hundreds of micrometers.

Statistical fluctuations of certain properties (e.g. the positions of impurities in semiconductor heterostructures) play an important role in mesoscopic systems yielding to the notion that two mesoscopic samples are not identical though they may belong to an ensemble which is describable in a statistical manner. The interest 


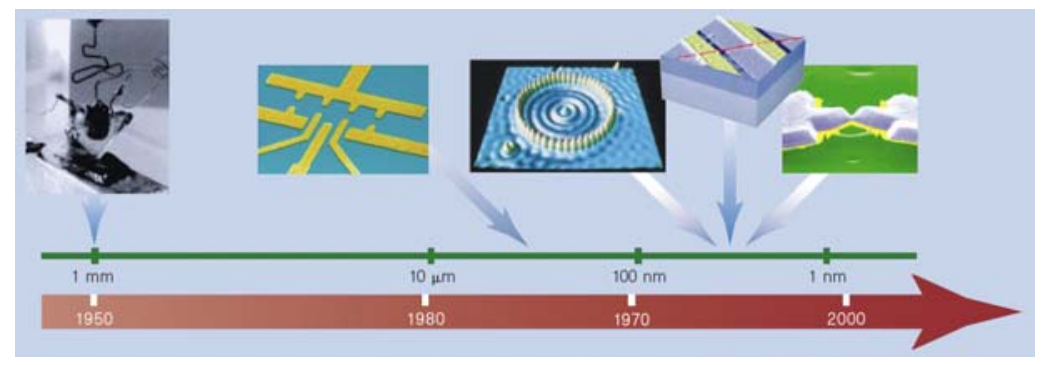

Figure 2.1: Progressive Miniaturization of electronic components. The length scale reached by technology has dropped steadily from the millimeter scale of the early 1950s to the present-day atomic scale. The representative devices, from left to right, are: the first transistor, a quantum-dot turnstile, a copper 'quantum corral', a carbon-nanotube transistor, and the latest - a one-atom point contact. 34

in studying mesoscopic systems is not only in order to understand the macroscopic limit and how it is achieved, by, say, building up larger and larger clusters to go from the single molecule to the bulk, but a variety of novel phenomena were found in the last decades that are intrinsic to mesoscopic systems. An example of a novel phenomenon are fractal conductance fluctuations which we will encounter in Sec.4.1. Progressive miniaturization e.g. of semiconductor devices leads to the fact, that mesoscopic phenomena become more and more important in contemporary nanotechnology (see Fig. 2.1). Many of the usual rules (such as Ohm's law and the rules for addition of resistances) are different and much more complicated.

A mesoscopic system is, in practice, always, at least weakly, coupled to a much larger system, via phonons, many-body interactions etc. Sometimes such a coupling can be controlled. Ideally, one would like to interpolate between open and closed systems by varying some coupling strength. In Chap. 3 we will analyze Bose-Einstein condensates in optical lattices described (in the mean-field limit) by the discrete nonlinear Schrödinger equation, where the coupling to the outside is realized by a complex dissipative term, which can be varied numerically and realized experimentally. This enables, besides possible technological applications, fundamental tests of quantum mechanics and statistical physics.

\subsection{Transport and Diffusion in Phase Space}

In the theory of transport in dynamical systems, the phase space volume flux plays an important role. Given a volume $V$ in phase space with surface $\delta V$. The volume of the trajectories leaving the volume $V$ per time unit is the flux out of $V$. For Hamiltonian systems the ingoing flux equals the outgoing flux. For dynamical systems discrete in time, the flux is calculated such that one iteration of the map relates to one time unit. Starting an ensemble of $N$ trajectories in a volume $V$ and calculating the staying probability $P=N(t) / N$, i.e. the relative number of trajectories remaining inside the volume at a time $t$, then for a fully chaotic system

$$
P(t) \propto \exp (-\gamma t)
$$




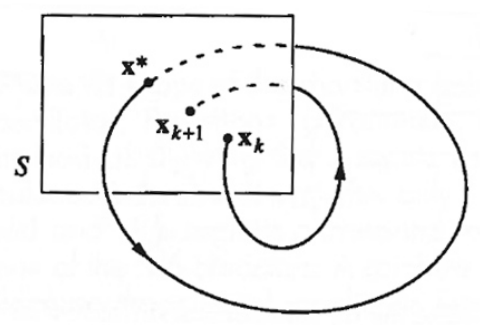

Figure 2.2: The Poincaré map $P$ is a mapping from a $n-1$ dimensional surface of section $S$ to itself, obtained by following trajectories from one intersection with $S$ to the next. If $x^{\star}$ is a fixed point of $P$, i.e. $P\left(x^{\star}\right)=x^{\star}$, then it is a closed orbit for the $n$-dimensional system $\dot{x}=f(x)$. [38

with rate $\gamma$. In contrast, in a system with a mixed phase space, consisting of regular islands embedded in the chaotic sea, the staying probability $P(t)$ decays algebraically

$$
P(t) \propto t^{-\delta}
$$

with exponent $\delta$. The algebraic behavior has its origin in the stickiness of trajectories in the vicinity of the regular islands (tori), see e.g. [35]. Chaotic trajectories in the vicinity of the islands can get caught for long times in a scale-free manner in the hierarchy of cantori. Every island is encircled by cantori, which are partial barriers in phase space that the orbit can penetrate. The deeper the orbit enters into the hierarchy of nested cantori, the longer it remains trapped, before it can leave the chaotic sea 35 37.

A very useful tool to investigate dynamical systems, especially when considering transport and diffusion in phase space, is the Poincaré map. Consider an $n$-dimensional system $\dot{x}=f(x)$. Choose an $n-1$ dimensional surface of section $S$, also referred to as Poincaré section, such, that $S$ is transverse to the flow in phase space, i.e. all trajectories starting on $S$ flow through it, not parallel to it (Fig. 2.2). Let $x_{k} \in S$ be the $k$ th intersection, then the Poincaré map is defined by

$$
x_{k+1}=P\left(x_{k}\right) .
$$

The Poincaré section is the generalization of a stroboscopic view of the dynamics in $n$ dimensions, and is most illustrative for $n=3$ as a surface of section $S$ can then be plotted in the 2-dimensional plane. When the mathematical formulation of a complex high dimensional system can be reduced to an appropriate set of a small number of relevant variables or degrees of freedom, the Poincaré map can give valuable information about the dynamics of the system. A great variety of area-preserving chaotic maps are investigated thoroughly in literature [35]. A paradigmatic model for mixed and chaotic phase space dynamics is the standard map which we will describe in the following.

\subsubsection{Standard Map}

The Standard map (also known as the kicked rotator) was introduced by Chirikov in the 1970s [39] and is an area-preserving chaotic map for two canonical dynamical 


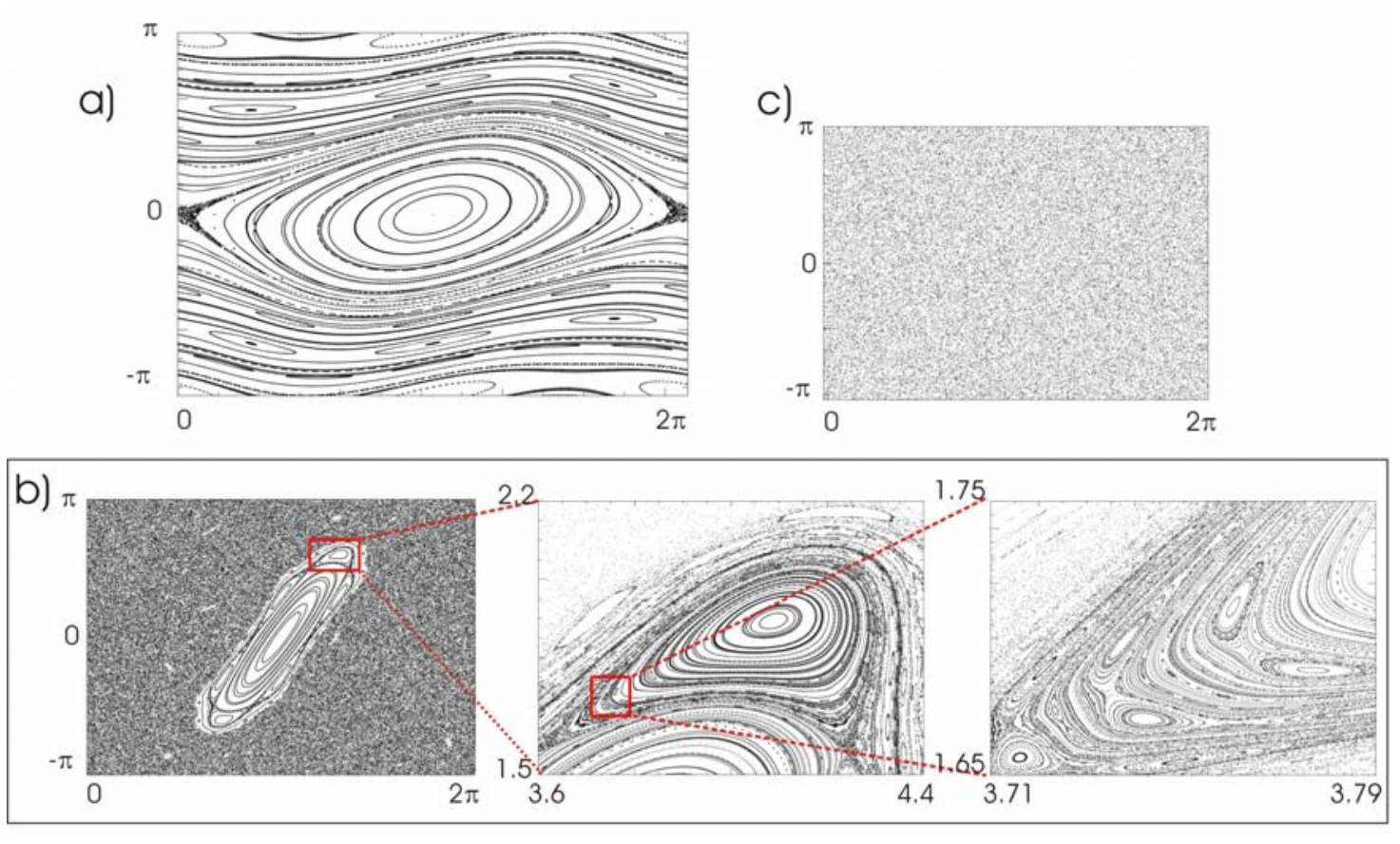

Figure 2.3: The KAM route to chaos generated with the standard map. (a) For relatively small nonlinearity $(K=0.55)$ many horizontally oriented KAM tori can be seen. (b) Mixed phase space $(K=3.5)$ with islands embedded in the chaotic sea. The enlargements demonstrate the hierarchical structure and immense complexity of a mixed phase space. (c) Fully chaotic phase space $(K=8)$. Shown is a single trajectory iterated for 50000 time steps that explores the whole phase space area.

variables, e.g. momentum and angle $(p, \theta)$. It is defined by the equations:

$$
\begin{aligned}
p_{n+1} & =p_{n}+K \sin \theta_{n} \\
\theta_{n+1} & =\theta_{n}+p_{n+1}
\end{aligned}
$$

Due to the periodicity of $\sin \theta$, the dynamics can be considered on a cylinder (by taking $\theta \bmod 2 \pi$ ) or on a torus (by taking both $\theta, p \bmod 2 \pi$ ). The map is generated by the time dependent Hamiltonian

$$
H(p, \theta, t)=\frac{p^{2}}{2}+K \cos \theta \sum_{n=0}^{\infty} \delta(t-n T),
$$

where for simplicity we will set the period of the kicks $T=1$. The dynamics is given by a sequence of free propagations interleaved with periodic kicks. The standard map goes through the whole KAM route to chaos in dependence of the nonlinearity parameter $K$ [40]: From integrable $(K=0)$ via a mixed phase space to fully chaotic $(K \gtrsim 7)$. In Fig. 2.3 we are iterating a number of different initial conditions for a long time. If the initial condition is on an invariant quasiperiodic torus, it traces out the closed curve corresponding to the torus. If the initial condition yields a chaotic orbit, then it will wander throughout an area densely filling that area. We see that for a relatively small perturbation $K=0.55$, there are many KAM tori running horizontally from $\theta=0$ to $\theta=2 \pi$ (Fig. 2.3a). These tori are those that 
originate from the nonresonant tori of the unperturbed system $(K=0)$ and have survived the perturbation (see the KAM theorem e.g. described in [35]). We also see tori that are created by the perturbation and nested around elliptic periodic orbits originating from the resonant tori. In Fig. $2.3 \mathrm{~b}$ the standard map for $K=3.5$ is shown, which exhibits a hierarchical mixed phase space consisting of regular islands embedded in the chaotic sea. Fig. 2.3c depicts a fully chaotic phase space explored by a single trajectory $(K=8)$.

Is it possible to describe aspects of the dynamics of complex (experimental) systems with such a simple map? The Hamiltonian Eq. 2.2 itself describes the motion of a simple mechanical system called a kicked rotator. This is made by a stick that is free of the gravitational force, which can rotate frictionless in a plane around an axis located in one of its tips, and which is periodically kicked on the other tip. The variables $\theta_{n}$ and $p_{n}$ respectively determine the angular position of the stick and its angular momentum after the $n$-th kick (see e.g. [41] for an experimental realization of the kicked rotator in atom optics).

However, much more crucial for our investigations will be, that the standard map contains the important characteristics to capture the main properties of a conservative system of two degrees of freedom. As such, the map can by viewed as directly corresponding to the Poincaré map at the boundary of e.g. a chaotic ballistic cavity, connecting it conceptually with experimental systems and making it a paradigmatic model for chaotic and mixed phase space dynamics.

We will encounter the standard map twice in this thesis: First, in Sec. 4.1 we shall see how Hamiltonian chaotic dynamics leads to fractal fluctuations in the conductance, as long as transport is at least partially conducted by chaotic dynamics. The underlying transport mechanism will be uncovered by analyzing an 'open' standard map (i.e. with absorbing boundaries). Second, in Chap. 3 we will relate a power law distribution of avalanches of BECs in optical lattices to a power law distribution of island sizes in a hierarchical mixed phase space. To this end, we will investigate the distribution of island sizes in the standard map indicating a power law as a generic feature of low-dimensional systems with a hierarchical mixed phase space.

\subsection{Fractal Analysis}

Both real and computer-simulated experiments in diverse systems in many fields of physics (and related sciences) often show characteristic structures or fluctuations. As these random structures can provide valuable information about the dynamical system under investigation, a statistical description and understanding of these structures is crucial.

Some random structures can be statistically rescaled by a self-affine transformation. In brief, a self-affine fractal can be described in general terms as having different scaling properties in different directions. In this thesis, fractal structures will appear twice, namely in: 1. fractal conductance fluctuations in mesoscopic systems and 2. fluctuations in human music rhythms.

A common quantity occurring in all the methods that will be discussed in the following is the Hurst exponent. Given a single-valued curve $f(x)$ of a function 


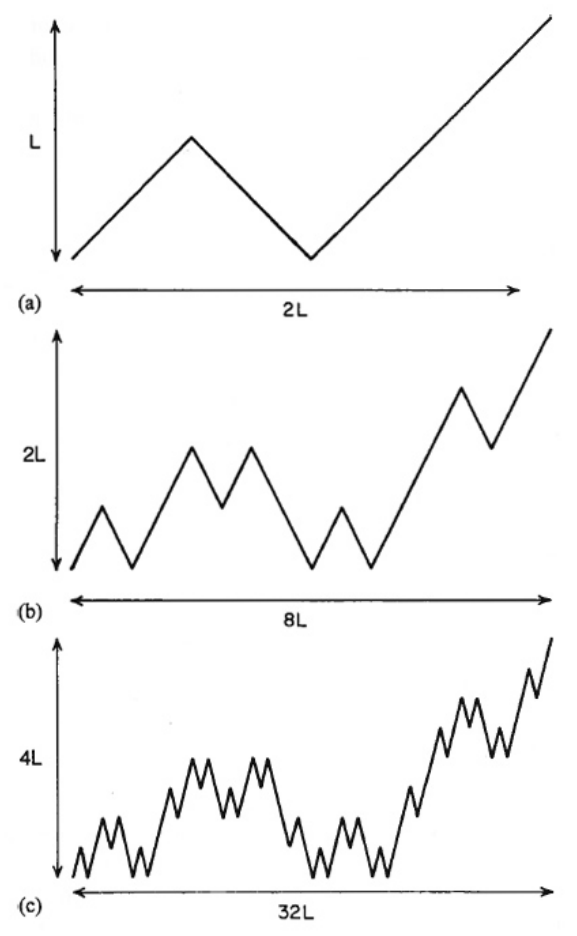

Figure 2.4: The construction of a simple, single-valued deterministic self-affine fractal curve. (a) The generator consists of four line segments of equal length. (b-c) In the second and third stage, each of the four line segments has been replaced by a replica of the generator. The horizontal length is increased by a factor of 4 (i.e. $s_{x}=4^{n}$ ), while the height is increased by a factor of 2 (i.e. $s_{y}=2^{n}$ ). In the asymptotic limit, the fractal curve $f(x)$ can be scaled onto itself by $s_{y}=s_{x}^{H}$ with Hurst exponent $H=1 / 2$. Figure taken from [42].

$f: \mathbb{R} \rightarrow \mathbb{R}$ that is generated by a self-similar construction process, where $n$ denotes the generation index (see Fig. 2.4 for an illustrative example). A self-affine curve $f(x)$ can be scaled onto itself by changing the horizontal length scale by a factor of $s_{x}=a^{n}$ while the vertical length is rescaled by a factor of $s_{y}=b^{n}$, so that $s_{y}=s_{x}^{H}$, where $H=\log _{b} a$ is the Hurst exponent. The fractal dimension is related to the Hurst exponent by

$$
D=2-H .
$$

In physics and related sciences, when fluctuations are found, typically the generator or the construction process in not known. Hence, the self-similar properties of the the fluctuations obtained are investigated in a statistical manner, for which a variety of methods exists. In the following, we will overview several methods which are used to analyze fractal properties of fluctuations. For a detailed description see e.g. [42], a comparison of the methods is drawn in [43, 44].

\subsubsection{Comparison of Different Methods}

Box-Counting One of the most prominent approaches of fractal analysis is the box-counting method. We will treat the $1+1$-dimensional case, generalization to higher dimensional manifolds is straightforward. Let $N(s)$ be the number of 
squares needed to cover the graph $G \in \mathbb{R} \times \mathbb{R}$ of a function $f: \mathbb{R} \rightarrow \mathbb{R}$, where $s$ is the length of one side of a square. If $N(s)$ behaves like a power law for small enough $s$, the box-counting dimension $D_{b o x}$ is defined as

$$
D_{b o x}:=\lim _{s \rightarrow 0}-\frac{\ln N(s)}{\ln s} .
$$

However, when applying the box-counting method numerically, caution has to be taken: Tests with fractal curves, where the Hurst exponent is known analytically (e.g. fractional Brownian motion or the Weierstrass-Mandelbrot series) show that the box-counting estimates are by far not the best and that other methods prove to be much more reliable [43. In contrast, the box counting is very useful in the analytical estimation of the fractal dimensionality of $n$-dimensional structures, notably when the generator or the underlying construction rule that leads to the structure is known. We will apply the box counting method analytically to a sequence of random transmission lobes in Sec. 4.1.5.3. For numerical estimates of the fractal dimension of conductance curves in Sec. 4.1, however, more suitable and reliable methods will be used as described in the following.

Variation Method and "Meakin Method" Given a mapping $f: \mathbb{R} \rightarrow \mathbb{R}$. The variation method, described in [43], is based on the calculation of the maximum variation $v\left(x_{0}, s\right)$ in a curve $f(x)$ within a distance $s$ of a point $x_{0}$ :

$$
v\left(x_{0}, s\right)=[\sup f(x)-\inf f(x)]_{\left|x_{0}-x\right|<s} .
$$

The "variation" $V(s, f)$ of $f(x)$ is defined as

$$
V(s, f)=\int_{0}^{s_{\max }} v\left(x_{0}, s\right) d x_{0}
$$

and the Hurst exponent is given by

$$
H=\lim _{s \rightarrow 0} \frac{\ln V(s)}{\ln (s)} .
$$

A similar method is proposed by Meakin [42] which consists simply of the height difference correlation function. A name was not found in literature, hence it will be called the "Meakin method" in this thesis. The idea behind the method originates in the observation, that in many important cases, a random self-affine fractal can be viewed of consisting of fluctuations about a straight reference line, in this case given by the constant mean value. In this perspective, the Hurst exponent characterizes the relationship between the height differences of pairs of points $\left(x_{1}, f\left(x_{1}\right), x_{2}, f\left(x_{2}\right)\right)$ of $f(x)$ with respect to that reference line. For a self-affine curve we find

$$
<\left|f\left(x_{1}\right)-f\left(x_{2}\right)\right|>_{\left|x_{1}-x_{2}\right|=s} \sim s^{H} .
$$

We tested both methods using the Weierstrass-Mandelbrot series and fractional Gaussian noise, where the Hurst exponent is known analytically. Both methods have shown to be a much more reliable tool than e.g. the box-counting method to numerically determine the fractal dimension of a graph $G \in \mathbb{R} \times \mathbb{R}$ and can be implemented very efficiently. We will use these methods to estimate the fractal dimension of conductance curves (Sec. 4.1). 
Detrended Fluctuation Analysis (DFA) The method of detrended fluctuation analysis, proposed in [45], has proven useful in revealing the extent of longrange correlations in time series. Similar to the Meakin method, fluctuations over a reference line are measured. However, in contrast to the Meakin method, the reference line is given by the local trend, i.e. the data is divided into boxes and detrended locally. More specifically, DFA involves a detrending of the data in the boxes using a polynomial of degree $m$, e.g. for linear and quadratic detrending the method is referred to as DFA1 and DFA2 respectively. We will describe DFA1, extension to DFA2, DFA3 etc. is straightforward.

Given a time series $f(t)$ of total length $N$ to be analyzed. First, the time series is integrated yielding

$$
y(k)=\sum_{t=1}^{k} f(t) .
$$

The integrated time series is divided into boxes of equal length, $s$. In each box, a least squares line $y_{s}(k)$ is fitted to the data (representing the linear trend in that box), see Fig. 4.20. Next, we detrend the integrated time series, $y(k)$, by subtracting the local trend, $y_{s}(k)$, in each box. The root-mean-square fluctuation of this detrended time series is calculated by

$$
F(s)=<y(k)-y_{s}(k)>=\sqrt{\frac{1}{N} \sum_{k=1}^{N} y(k)-y_{s}(k)}
$$

This computation is repeated over the time scales (box sizes) of interest to characterize the relationship between the average fluctuation $F(s)$, and the box size $s$. A linear relationship on a log-log plot indicates the presence of power law (fractal) scaling $F(s) \sim s^{\alpha}$ with scaling exponent $\alpha$. For fractional Gaussian noise the exponent $\alpha$ is equal to the Hurst exponent $\alpha=H$, while for fractional Brownian motion $\alpha=H-1$. We will use DFA to analyze error time series of rhythmic music sequences played by humans in Sec. 4.2 .

\subsection{Discrete Breathers}

An important and exciting feature appearing in the frame of nonlinear lattices are discrete breathers (DBs), which we will encounter in Chap. 3. The following working definition is taken from [46] (see as well [47, 48] for an overview):

"Discrete breathers (DB) or intrinsic localized modes are spatially localized, time-periodic, stable (or at least long-lived) excitations in spatially extended perfectly periodic discrete systems."

The phenomenon of localization of, e.g. energy or particles is well known in solid state physics. Typical examples are the localized vibrational phonon modes around impurities or defects in crystals and Anderson localization of electrons in disordered media. Localization is usually perceived as arising from external disorder, e.g. in the case of Anderson localization, that breaks the discrete translational invariance of a perfect crystal lattice. In contrast, in the late 1980s it was found that intrinsic 

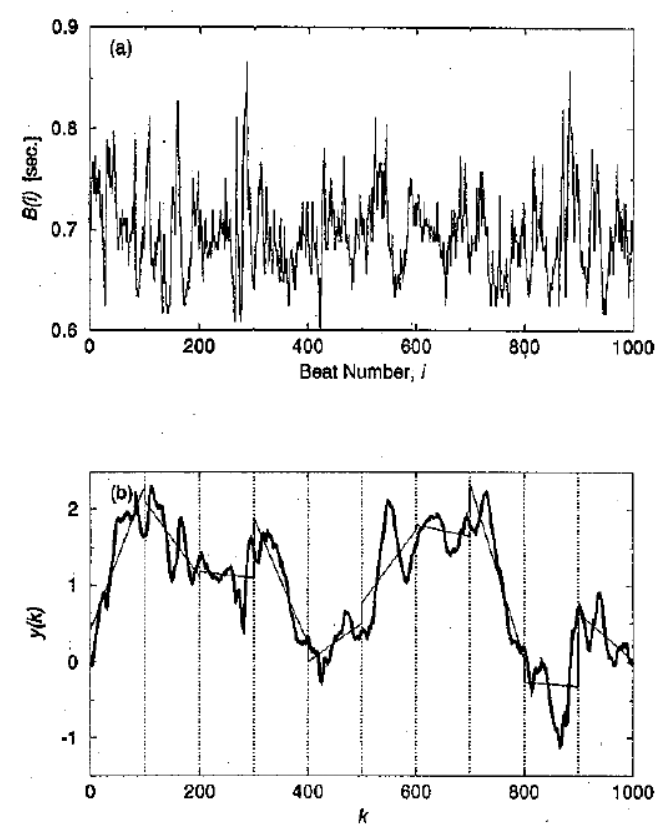

Figure 2.5: Illustration of DFA 1 (linear detrending). (a) A time series $B(i)$, here consisting of 1000 interbeat intervals of the human heartbeat. (b) Illustration of the detrending of the integrated time series $y(k)=\sum_{i=1}^{k}\left(B(i)-B_{\text {ave }}\right)$, where $B(i)$ is the interbeat interval shown in (a). The vertical dotted lines divide the axis of abscissae into boxes of size $s=100$. The solid straight line segments represent the local (here: linear) trend estimated in each box. Figure taken from [45].

localized modes (also called discrete breathers) are, in fact, typical excitations in perfectly periodic but sufficiently nonlinear systems [48 51]. DBs were observed in a variety of systems, such as Josephson-junction arrays [52], micromechanical systems [53], photonic crystals [54], nonlinear waveguide arrays [55], $\alpha$ helix of a protein [56] and spins in antiferromagnetic solids [57].

Let us shortly review the history of DBs. It reaches back to the famous E. Fermi, J. Pasta and S. Ulam, who conducted in 1953 the following numerical experiment later known as the FPU problem: Imagine a perfectly periodic vibrating string, where the equations of motion include a nonlinear term. One of the equations they investigated was

$$
\ddot{x}_{i}=\left(x_{i+1}-x_{i-1}-2 x_{i}\right)+c\left(\left(x_{i+1}-x_{i}\right)^{2}-\left(x_{i}-x_{i-1}\right)^{2}\right),
$$

where $x_{i}$ denotes the displacement of the $i$-th point from its original position and $c$ is the coefficient in the nonlinear (quadratic) force between neighboring mass points and $i=1 \ldots 64$. They found that the behavior of the system was quite different from what intuition had led them to expect. The expectation was that after many iterations, the system would exhibit thermalization, an ergodic behavior in which the influence of the initial modes of vibration fade and the system becomes more or less random with all modes excited more or less equally. Instead, the system exhibits an oscillatory ("breathing-like") behavior [59]:

"The results show very little, if any, tendency toward equipartition of energy among the degrees of freedom." 

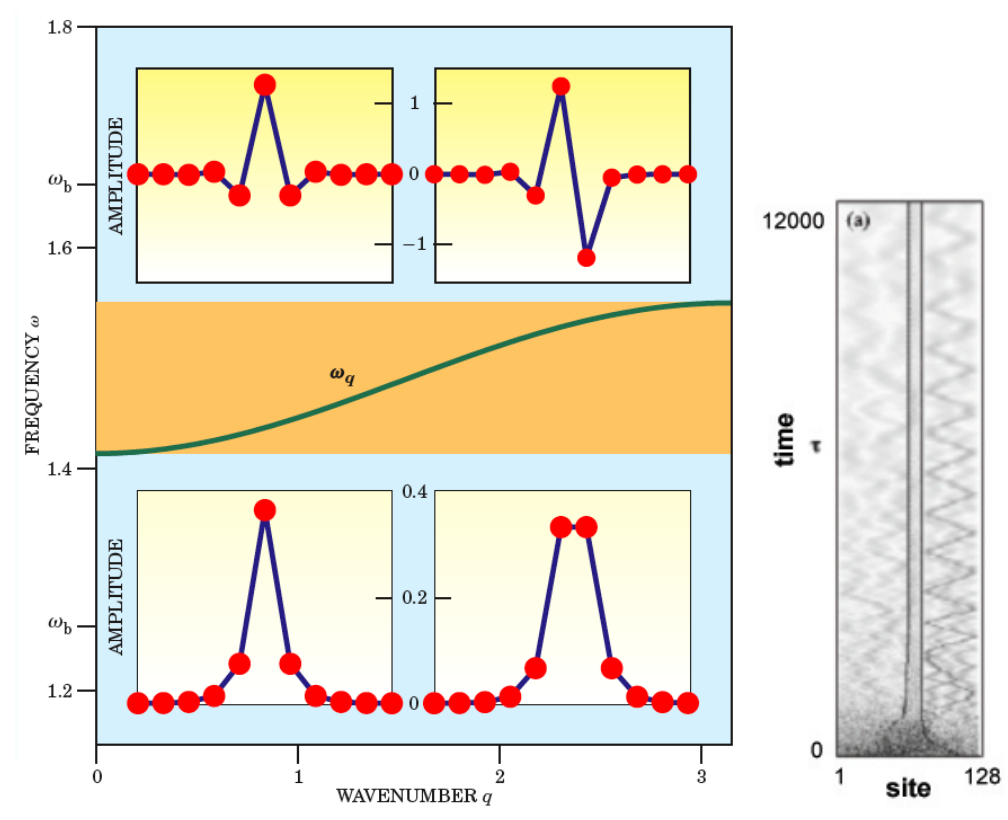

Figure 2.6: (left) Frequency versus wavenumber plane shows the spectrum of linear oscillations and two isolated frequencies $\omega_{b}$ outside the linear spectrum corresponding to discrete breathers [46]. The red circles indicate the amplitudes (e.g. particle displacements) for the DB solution. (right) The discrete nonlinear Schrödinger equation (see Sec. 3.2 rigorously exhibits discrete breathers [58].

This (at first sight) puzzling computer experiment leads to the question: How can localization arise in a perfectly periodic lattice and what makes a DB stable? Linear excitations - be they electrons or phonons - moving through a solid will experience a periodic energy potential, which implies by the Bloch theorem the existence of 'forbidden' and 'allowed' bands of frequency and velocity for their motion. Linear excitations can propagate through the solid only in the allowed bands which have a highest and a lowest frequency. The situation is different for nonlinear excitations. As can be seen from the simple one dimensional pendulum, the frequency is independent of the amplitude when linearizing the equations of motion, but does depend on the amplitude in the nonlinear (high amplitude) regime. If a large amplitude (and hence nonlinear) excitation is created - a possible candidate for a discrete breather - it's frequency can lie outside the allowed band of linear excitations (see Fig. 2.6). The highest frequency of the allowed band is determined by the degree of discreteness of the lattice: The larger the lattice constant, the smaller the highest frequency of the linear band. If all harmonics of the DB frequency lie outside (above) the allowed band, then the DB cannot couple to linear excitations and is therefore stable against decaying into them. To summarize, a DB is a localized oscillatory excitation that is stabilized against decay by the discreteness of a nonlinear periodic lattice. The stability of DBs in BECs will play a crucial role in Chap. 3.

A quantity related to DBs is the Peierls-Nabarro barrier, which is given by the energy difference $\left|E_{c}-E_{b}\right|$, where $E_{c}$ and $E_{b}$ are the energies for a DB centered at a lattice site or between two lattice sites [60, 61, see Fig. 2.7. 

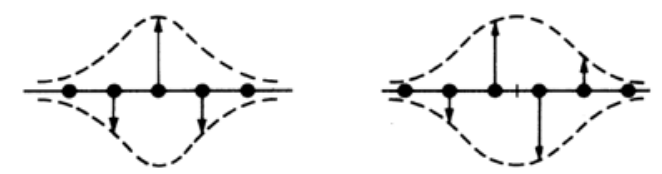

Figure 2.7: Peierls-Nabarro barrier: Energy difference between the states where a DB is centered at a lattice site (left) or between two lattice sites (right) [60].

Finally, we end this chapter with a note on the nomenclature adopted from [62]. In literature one encounters terms such as polarons, discrete solitons, discrete breathers, self-trapped state, intrinsic localized modes etc., that are used many times interchangeably (which reflects that several historical paths lead to the discovery of the same phenomenon) and in ways that may lead to confusion. For simplicity, throughout this work, the term discrete breather will be used. 


\section{Chapter 3}

\section{Avalanches of BECs in Optical Lattices}

One of the most fascinating experimental achievements of the last decade was unambiguously the realization of Bose-Einstein Condensation (BEC) of ultra-cold atoms in optical lattices (OLs) [6, 63 66]. Experimentalists have achieved an extraordinary level of control over BECs in optical traps in the past decade, which allows for the investigation of complex solid state phenomena [6-13] and the emerging field of "atomtronics" promises a new generation of nanoscale devices such as an atom laser. The atom laser, a bright, coherent matter wave derived from a Bose-Einstein condensate holds great promise for precision measurement and for fundamental tests of quantum mechanics. It is therefore both of fundamental and technological importance to understand the dynamics and transport properties of BECs in OLs. We ask the following question: What are the transport properties of BECs in leaking optical lattices and can we understand the statistics of the outgoing flux of ultracold bosons?

We study the decay of an atomic BEC population $N(\tau)$ from the leaking boundaries of an optical lattice using a mean field description provided by the discrete nonlinear Schrödinger equation (DNLS). The DNLS, described in detail in Sec. 3.2. is a lattice equation that contains a nonlinearity $\Lambda$. An exciting feature appearing in nonlinear lattices is the existence of discrete breathers (DBs), which are spatially localized, time-periodic and stable (or at least long-lived) excitations. DBs emerge due to the nonlinearity and discreteness of the system (Historically, the Fermi-Pasta-Ulam problem lead to the discovery of discrete breathers in the 1950s, see Sec. 2.4 for an introduction). DBs were observed in various experimental setups [3, 52, 55, 67, 74] while their existence and stability were studied thoroughly during the last decade [46, 48, 49, 51, 75, 79]. It was shown that they act as virtual bottlenecks which slow down the relaxation processes in generic nonlinear lattices [51, 78, 81]. Further works [82,86] established the fact that absorbing boundaries can take generic initial conditions towards DBs.

In the DNLS with dissipation at the ends of the lattice, we find that the dynamics evolves into the population of discrete breathers for a nonlinearity larger than a threshold $\Lambda>\Lambda_{b}$ preventing the atoms from reaching the leaking boundaries. We show that collisions of other lattice excitations (e.g. a moving breather, see Sec. 2.4 with the outermost DBs result in avalanches, i.e. steps in $N(\tau)$, which for 
a whole range of $\Lambda$-values follow a scale-free distribution [28]

$$
\mathcal{P}(J=\delta N) \sim 1 / J^{\alpha}
$$

characterizing systems at a phase transition. We will see that the scale-free behavior of $\mathcal{P}(J)$ reflects the complexity and the hierarchical structure of the underlying classical mixed phase space of the trimer. A theoretical analysis of the mixed phase space of the system indicates that $1<\alpha<3$ in agreement with our numerical findings.

We propose an order parameter to describe the observed phase transition. Though we do have clear numerical evidence concerning the phase transition, an understanding of the phase transition together with an analytical expression for $\Lambda_{b}$ is still an open and fascinating question and work in progress [87]. The collision process of a stationary breather with a moving breather is analyzed analytically and numerically in a reduced system consisting of 3 lattice sites, called the nonlinear trimer [29] (by means of the local ansatz [49] described in Sec. 3.6). We point out that although our focus is given to atomic BECs, our results are also relevant in a large variety of contexts (whenever the DNLS is adequate), most prominently being the light emittance from coupled nonlinear optical waveguides [1 5, 54, 55, 74, 88, 92], see Sec. 3.9 for more details on discrete breathers in optical waveguide arrays.

\subsection{Experimental Setup}

We consider the statistics of emitted ultracold atoms from an OL with leakage at the edges. Typically, ultracold atoms are stored in magnetic dipole traps, that make use of the interaction between an induced dipole moment in an atom and an external electric field provided by a laser. A periodic potential can then be formed by overlapping two counter-propagating laser beams as shown in Fig. 3.1. The magnetic field gives rise to a harmonic trapping potential which confines the condensate in an array of tightly confining $1 \mathrm{D}$ potential tubes, for our purposes with its long axis oriented perpendicular to the gravitational force. Along the 1D tubes, a periodic potential can be created (again with two counter-propagating laser beams) leading to a 1D optical lattice (Fig. 3.2 a). The depths of the optical potential, i.e. the tunneling amplitude between the lattice sites, can be varied by changing the intensity of the laser light.

The leakage can be realized experimentally by applying two separate continuous microwave fields or Raman lasers at the edges of the sample to locally spin-flip the atoms inside the BEC to an untrapped state [58, 86, 93, 94] The spinflipped atoms do not experience the magnetic trapping potential, and hence they are released through gravity at the ends of the OL ( Fig. $3.2 \mathrm{p}$ ). An experimental realization of a continuous output of atoms is shown in Fig. 3.3 a, where a field with frequency $\nu$ induces transitions from the magnetically trapped $\mid F=2, m_{F}=2>$ state to the untrapped $\mid F=2, m_{F}=0>$ state via the $\mid F=2, m_{F}=1>$ state. Here,

\footnotetext{
${ }^{1}$ Spatially localized microwave fields focused below the wavelength can be obtained at the tip of tapered waveguides.
} 
$F$ denotes the total angular momentum and $m_{F}$ is the magnetic quantum number. The resonance condition reads $\frac{1}{2} \mu_{B}|B(r)|=h \nu$, where $\mu_{B}$ is the Bohr magneton. An experimental realization of the time-resolved counting of the released atoms is shown in Fig. 3.3b [94]. Thus, an accurate monitoring of the decay process of the atomic population can be utilized to probe the dynamical properties of BECs inside an optical lattice.
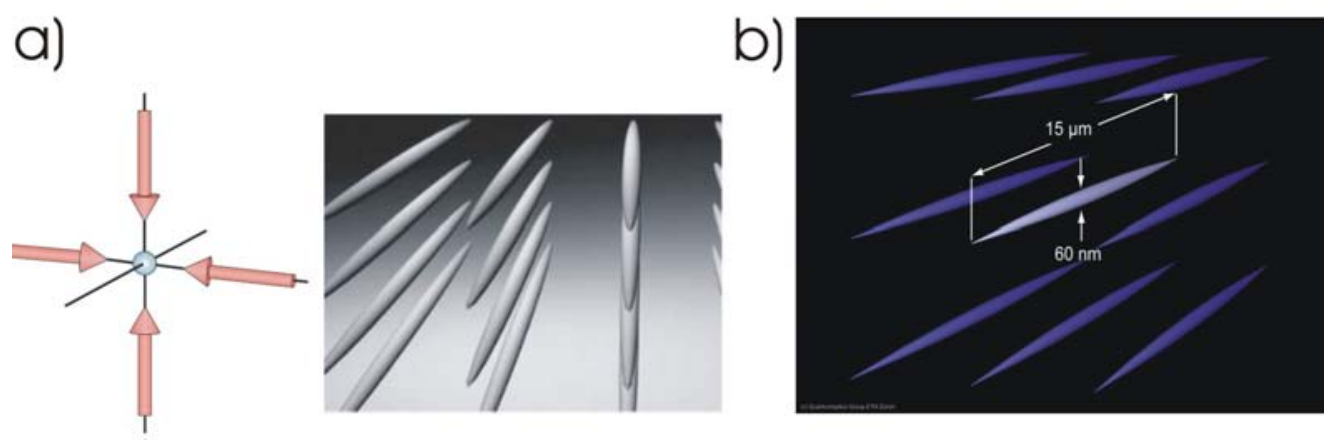

Figure 3.1: a) Optical lattice potentials formed by superimposing two orthogonal standing waves [66]. b) For a 2D optical lattice, the atoms are confined to an array of tightly confining 1D potential tubes (in this picture of $15 \mu \mathrm{m}$ length and $60 \mathrm{~nm}$ width). The picture is taken from www.quantumoptics.ethz.ch.

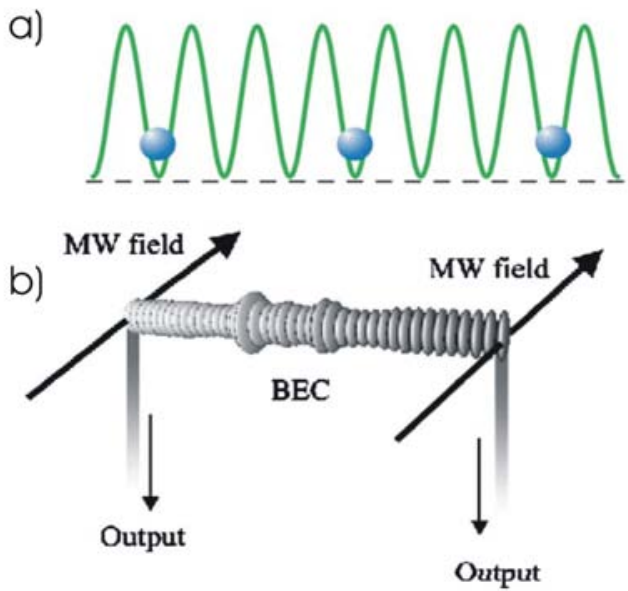

Figure 3.2: a) Illustration (taken from [66]) of BECs loaded in an optical lattice. The standing-wave interference pattern creates a periodic potential in which the atoms move by tunnel coupling between the individual wells. b) Schematic realization of leakage at the two edges of the lattice using continuous microwave or Raman lasers to spin-flip atoms that reach the edges to a untrapped state (Figure taken from [86]). Thus, the atoms at the edges do not experience the magnetic trapping and hence are released through gravity. The released atoms are then measured at the detectors. 

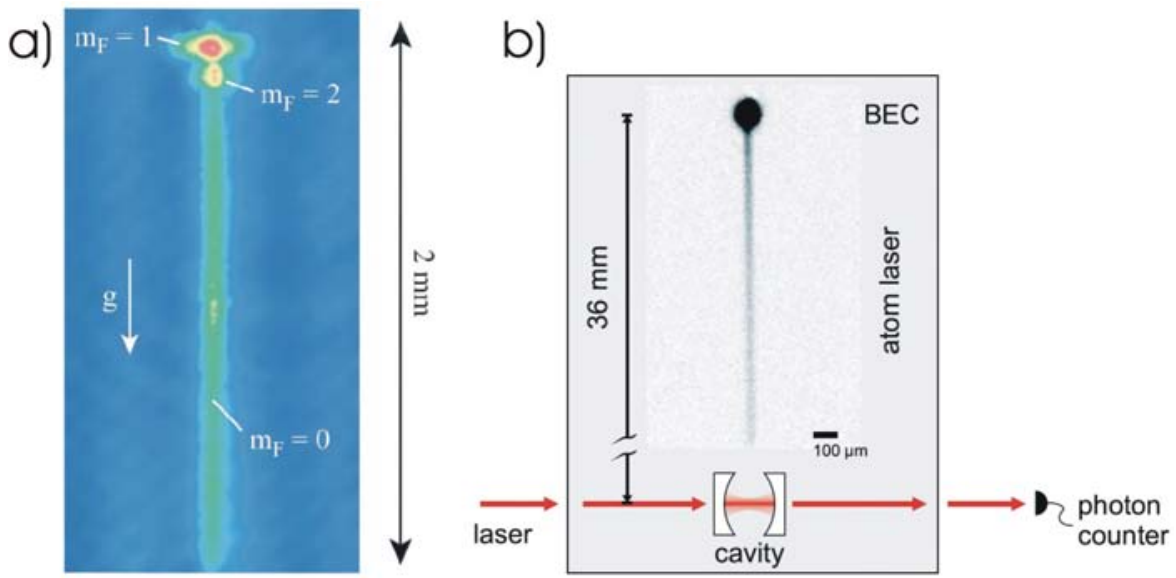

Figure 3.3: a) Experimental realization of a continuous atom laser output (figure taken from [93]): A collimated atomic beam is derived from a Bose-Einstein condensate over a $15 \mathrm{~ms}$ period of continuous output coupling. A fraction of condensed atoms has remained in the magnetically trapped $\mid F=2, m_{F}=2>$ and $\mid F=2, m_{F}=1>$ state. b) Report of an experiment that enables counting of single atoms of an atom laser taken from 94 . A schematic of the experimental setup is shown, where a continuous atom laser beam is released from a BEC. After dropping a macroscopic distance of $36 \mathrm{~mm}$, the atoms enter an optical cavity where single atoms in the beam are detected.

\subsection{Discrete Nonlinear Schrödinger Equation}

The simplest model that captures the dynamics of a dilute gas of bosonic atoms in a deep OL, with chemical potential small compared to the vibrational level spacing, is the Bose-Hubbard Hamiltonian. A few essential points will be mentioned here, see e.g. [95] for a detailed review.

In the case of weak interatomic interactions (superfluid limit) or a large number of atoms per well (so that the total number of atoms $N \sim \mathcal{O}\left(10^{4}-10^{5}\right.$ ) is much bigger than the number of wells $M$ ), a further simplification is available since the BECs dynamics admits a semiclassical (mean-field) description. The resulting semiclassical Hamiltonian that describes the dynamics is

$$
\mathcal{H}=\sum_{n=1}^{M}\left[U\left|\psi_{n}\right|^{4}+\mu_{n}\left|\psi_{n}\right|^{2}\right]-\frac{T}{2} \sum_{n=1}^{M-1}\left(\psi_{n}^{*} \psi_{n+1}+\text { c.c. }\right)
$$

where $n=1, \ldots, M$ is the index of the lattice site, $\left|\psi_{n}(t)\right|^{2} \equiv N_{n}(t)$ is the mean number of bosons at site $n, U=4 \pi \hbar^{2} a_{s} V_{\text {eff }} / m$ describes the interaction between two atoms at a single site ( $V_{\text {eff }}$ is the effective mode volume of each site, $m$ is the atomic mass, and $a_{s}$ is the $s$-wave atomic scattering length), $\mu_{n}$ is the on-site chemical potential, and $T$ is the tunneling amplitude. The "wavefunctions"

$$
\psi_{n}(t) \equiv A_{n}(t) e^{-i \phi_{n}(t)}
$$

with amplitudes $A_{n}(t)$ and phases $\phi_{n}(t)$ can be used as conjugate variables with 
respect to the Hamiltonian $i \mathcal{H}$ leading to a set of canonical equations

$$
\begin{aligned}
i \frac{\partial \psi_{n}}{\partial t} & =\frac{\partial \mathcal{H}}{\partial \psi_{n}^{*}} \\
i \frac{\partial \psi_{n}^{*}}{\partial t} & =-\frac{\partial \mathcal{H}}{\partial \psi_{n}}
\end{aligned}
$$

which upon evaluation yields the Discrete Nonlinear Schrödinger Equation (DNLS)

$$
i \frac{\partial \psi_{n}}{\partial \tau}=\lambda\left(\left|\psi_{n}\right|^{2}+\mu_{n}\right) \psi_{n}-\frac{1}{2}\left[\psi_{n-1}+\psi_{n+1}\right] ; \quad n=1, \ldots, M .
$$

Here, $\lambda=2 U / T$ is the nonlinearity and $\tau=T t$ is the normalized time.

The DNLS can be applied to a remarkably large variety of systems, examples include Davydov's model for energy transport in biomolecules, or the theory of local modes of small molecules [96] and within nonlinear optics it is a model of coupled nonlinear waveguides [1]. In particular this mathematical model describes (in the mean-field limit) the dynamics of a BEC in a leaking OL of size $M$ [97]. We will treat the repulsive case explicitly $(\lambda>0)$, however, the attractive case can be obtained via the staggering transformation $\psi_{n} \rightarrow\left(-1^{n}\right) \psi_{n}$ [48. To simulate the output coupling of atoms at the boundaries of our $1 D$ lattice, we supplement the standard DNLS with local dissipation terms at the two edges of the lattice [58, 86]. The resulting equation reads:

$$
i \frac{\partial \psi_{n}}{\partial \tau}=\Lambda\left|\psi_{n}\right|^{2} \psi_{n}-\frac{1}{2}\left[\psi_{n-1}+\psi_{n+1}\right]-i \gamma \psi_{n}\left[\delta_{n, 1}+\delta_{n, M}\right] ; \quad n=1, \ldots, M,
$$

where $\gamma$ is the dissipation rate and we defined an initial effective (rescaled) interatomic interaction per site

$$
\Lambda=\lambda \rho,
$$

with $\rho=N(t=0) / M$ being the initial average density of atoms in the OL, so that for different lattice sizes $M$, we maintain the same local dynamics by keeping $\Lambda$ constant. In Eq. 3.5 we have set $\mu_{n}=0 \forall n$, i.e. static disorder will not be treated in the following. The time $t$, the interatomic interaction $\lambda$, and the atom emission probability $\gamma$ describing atomic losses from the boundary of the OL are measured in units of the tunneling rate $T$. In an experimental setup, $T$ can be adjusted by the intensity of the standing laser wave field and the on-site interaction $U$ depends on the confining potential perpendicular to the tube in which the atoms move. Thus, the nonlinearity $\lambda$ can be varied experimentally.

\subsubsection{Estimating the Leakage Term}

In order to be able to compare with experiments, especially with BECs in leaking OLs, the dissipation rate $\gamma$ will be estimated within a mean-field approximation [86. Here, we consider the case of two output-coupler fields interacting with the atoms at the first and last lattice wells only. We can describe the output coupling through an external reservoir formed by an infinite number of states [86]. For optical input-output theory and in proposed atom laser theories that result in BornMarkov master equations, typically $\kappa(k)=$ const. is chosen (broadband coupling) 
[98], where the function $\kappa(k)$ describes the shape of the (output) coupling in $k$ space. For a broadband output coupling $\kappa$ the Born-Markov approximation leading to an exponentially decaying atomic density inside the BEC should satisfy [98

$$
\frac{\omega^{3 / 2}}{\pi \kappa^{2}} \sqrt{\frac{\hbar}{2 m}} \gg 1
$$

where $\omega$ is the $1 \mathrm{D}$ trapping frequency and $m$ is the atomic mass. And on the other hand, the characteristic decay time is given by

$$
t_{D}=\frac{1}{\pi \kappa^{2}} \sqrt{\frac{2 \omega \hbar}{m}}=\frac{\hbar / T}{\gamma},
$$

leading to

$$
\gamma=\frac{\pi \kappa^{2} \sqrt{\hbar m}}{T \sqrt{2 \omega}}
$$

Eq. 3.9 shows the proportionality between the dissipation rate $\gamma$ and the square of the coupling strength $\kappa$ and gives (together with Eq. 3.7) a condition on the magnitude of the dissipation rate $\gamma$ in order for the Born-Markov approximation to be valid:

$$
\frac{\hbar \omega}{2 \gamma T} \gg 1
$$

Using typical parameter values of experiments of BECs in optical lattices, which are $\hbar / T \approx 6 \times 10^{-4}$ and $\omega \approx 80 \mathrm{kHz}$ [7], the above condition is fulfilled up to $\gamma \approx 0.5$. The results for the leaking system reported below are for a dissipation rate of $\gamma=0.2$. Nevertheless, we have checked that the qualitative behavior is the same for other values of $\gamma<0.5$. For larger values of $\gamma$, non-Markovian terms have to be included in the description [99].

\subsection{Survival Probability: Avalanches}

Let us now study the decay and the statistical properties of the total atomic population inside the OL (also referred to as survival probability or total norm)

$$
\mathcal{N}(\tau)=\frac{N(\tau)}{N(0)}=\sum_{n=1}^{M}\left|\psi_{n}(\tau)\right|^{2},
$$

where we normalized the wave functions such that

$$
N(t=0)=1
$$

Its time derivative $-\frac{d N(\tau)}{d \tau}$ is equal to the outgoing atomic flux. In our numerical experiments we have used initial conditions with randomly distributed phases for the wavefunctions $\psi_{n}=A_{n} \exp \left(-i \phi_{n}\right)$, while $N_{n}(\tau=0)$ was taken to be almost constant with only small random fluctuations across the OL. The initial states were first "thermalized" during a conservative (i.e. $\gamma=0$ ) transient period of, typically, $\tau=500$. Only after this transient is completed, the dissipation at the lattice 
boundaries is switched on, leading to a progressive loss of atoms. The dynamical evolution is done through numerical integration by the Runge-Kutta-Fehlberg method with an accuracy such that for the largest system studied $(M=4096)$ deviations of $\mathcal{N}(\tau)$ from unity in a closed system $(\gamma=0)$ were less than $10^{-4}$ for the total time range studied $(t \leq 30000)$.

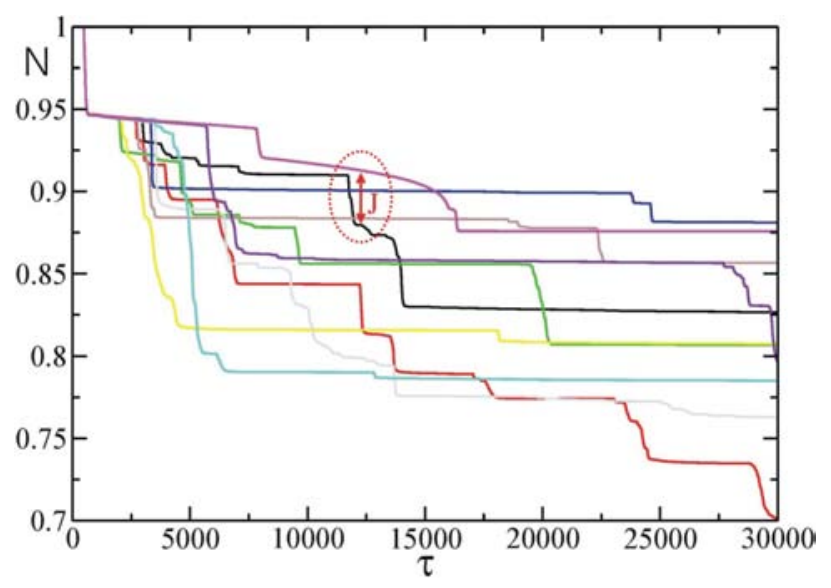

Figure 3.4: Representative realizations of atomic population decay exhibiting avalanches with jump size $J$. Shown is the survival probability $N(\tau)$ for various initial conditions. In the encircled region a single avalanche is marked.

In Fig. 3.4, we show the temporal evolution of $N(\tau)$ for various initial conditions for $\Lambda=1$ (see also [58, 86]). A striking feature is the appearance of jumps, indicating an avalanche-like behavior where a sudden burst of density (e.g. mass, number of atoms or energy) occurs. Our target is to analyze the distribution $\mathcal{P}(J)$ of these jumps $J$ for an ensemble of initial conditions and thus we have to analyze a considerable number of trajectories. To this end, we have defined the burst by a threshold $D^{*}$ in the derivative $D(\tau)=|d N(\tau) / d \tau|$ as shown in Fig. 3.5. In all cases studied, we had at least $10^{4}$ trajectories at fixed parameters for statistical processing. We have found that for a whole range of $\Lambda$ values the avalanches follow a scale-free distribution,

$$
\mathcal{P}(J) \sim J^{-\alpha}
$$

as demonstrated in Fig. 3.6. To understand the origin of the scale-free distribution $P(J)$, we will examine the dynamics of the atomic population $N_{n}(\tau)=\left|\psi_{n}(\tau)\right|^{2}$ inside the lattice in the next chapter.

\subsection{Dynamics of BECs}

In this section we investigate the dynamics of the atomic density of BECs inside the optical lattice. We will see that a complex interplay between discrete breathers and other lattice excitations is directly linked to the formation of avalanches.

In Fig. 3.7, we show the density plots that capture the dynamics of the leaking system (i.e. $\gamma \neq 0$ ) for some representative values of the rescaled nonlinearity $\Lambda$. The color represents the normalized (with respect to the original population) atomic population $N_{n}(\tau)=\left|\psi_{n}(\tau)\right|^{2}$ at each site. For small nonlinearity strengths 


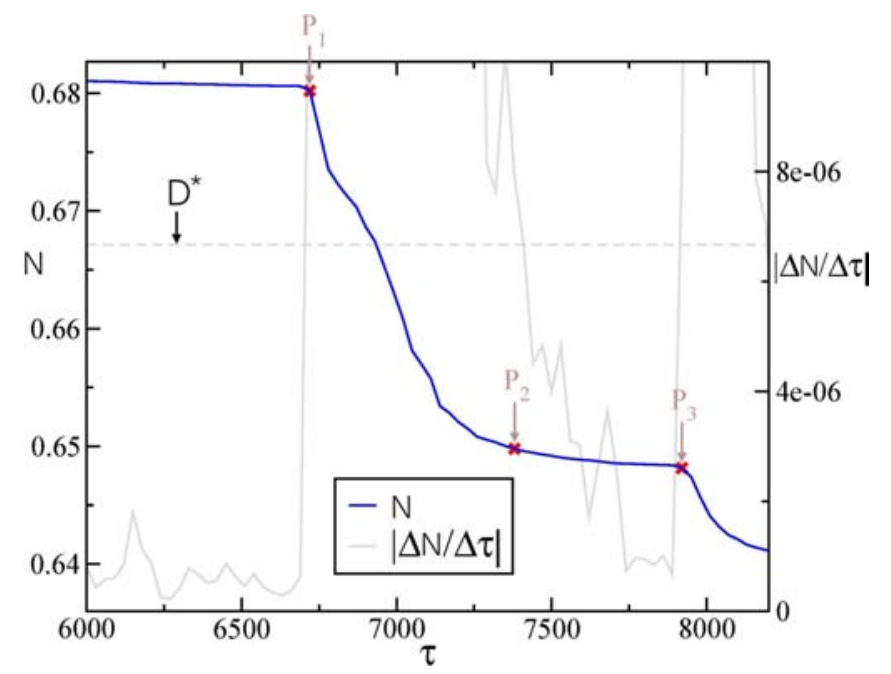

Figure 3.5: Definition of a jump in the total atomic population $N(\tau)$. Whenever $D(\tau)=$ $|d N(\tau) / d \tau|$ rises above $D^{*}$ we register a burst until it drops again below the threshold. In most of our calculations we have used $D^{*} \approx 10^{-5}$. However, we have checked that our numerical results for the distribution $\mathcal{P}(J)$ are stable for other choices of the threshold and for different sampling intervals of the $N(\tau)$ time-series.

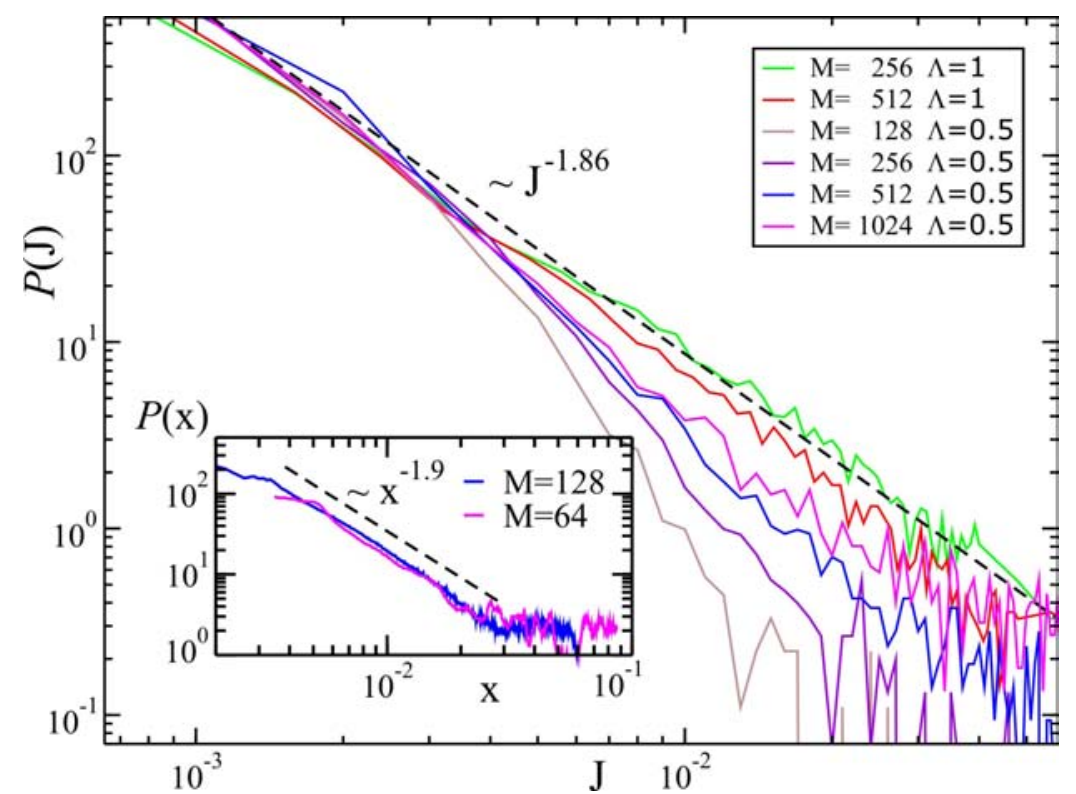

Figure 3.6: Distribution of avalanches $\mathcal{P}(\mathrm{J})$ for various system sizes $M$ and interatomic interaction strengths $\Lambda=0.5$ and $\Lambda=1$. In the former case we observe a convergence to a power law distribution $\mathcal{P}(\mathrm{J}) \sim J^{-\alpha}$ as the lattice size $M$ increases, while in the latter case the asymptotic distribution has already been reached for $M=512$. A least square fit yields $\alpha=1.86 \pm 0.04$ in agreement with the bounds $1<\alpha<3$ (see text). Inset: Power law distribution of norms $\mathcal{P}\left(\mathrm{x}=\left|\psi_{\mathrm{n}}\right|^{2}\right) \sim \mathrm{x}^{-\beta}$ for $\Lambda=1$. The best least square fit indicates that $\beta=1.9 \pm 0.05 \approx \alpha$. We set $\gamma=0.2$ in all cases.

$\Lambda \leq \Lambda_{b} \approx 0.15$ (Fig. 3.7 a), the system behaves as in the linear regime, i.e. the density is distributed uniformly across the whole lattice. In the opposite limit of $\Lambda \gg \Lambda_{b}$ (Fig. 3.7p) we observed the formation of the order of $M$ discrete breathers. 


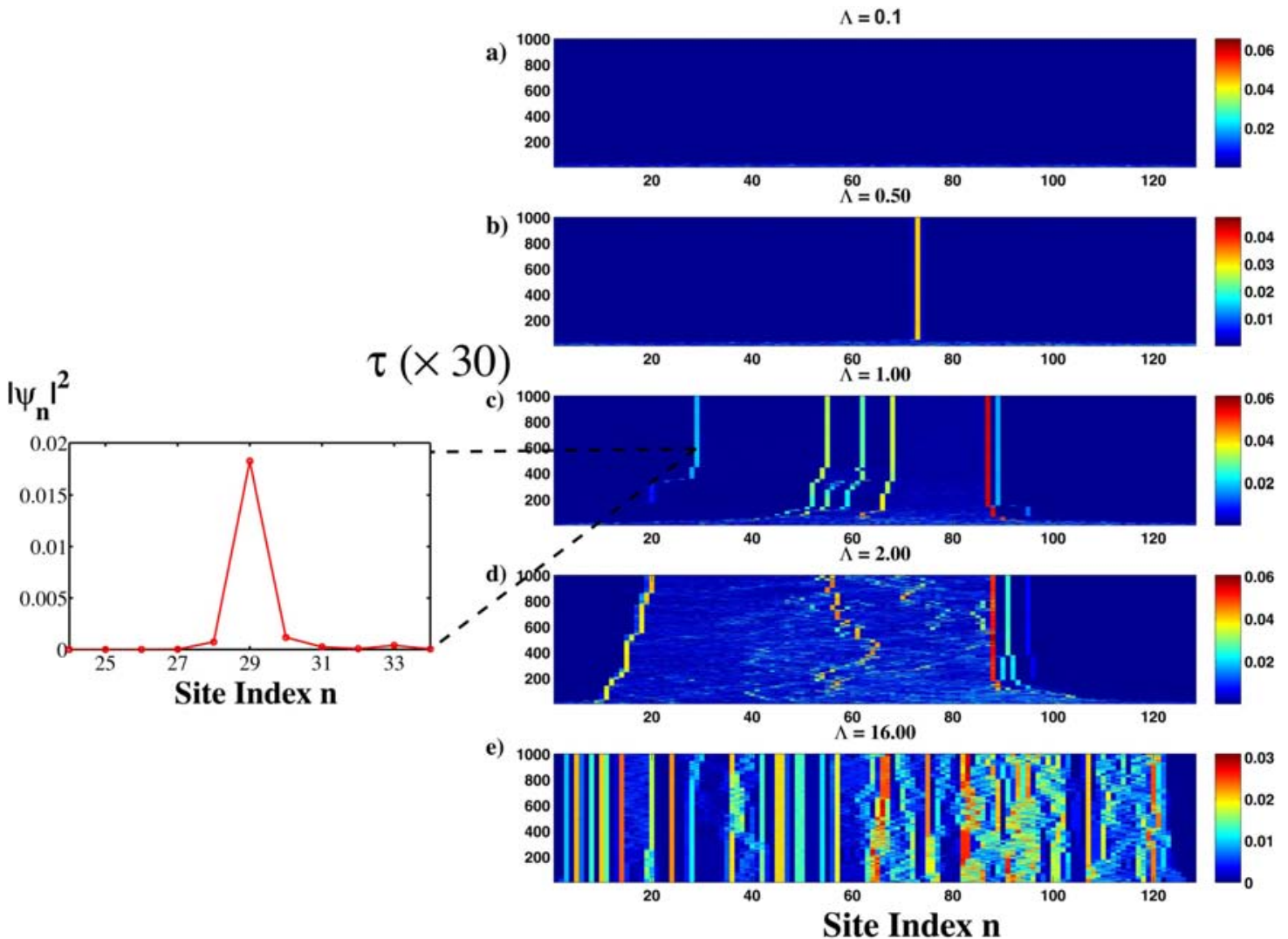

Figure 3.7: The right figure shows the evolution of the atomic population for a lattice of size $M=128$, dissipation rate $\gamma=0.2$ and various interatomic interaction strengths $\Lambda$ : (a) $\Lambda<\Lambda_{b}$ where no DBs are formed and the density is distributed uniformly across the whole lattice while for (b) $\Lambda>\Lambda_{b}$, the first breather appears. At (c) $\Lambda=\mathcal{O}(1)>\Lambda_{b}$ stationary breathers co-exist with moving breathers, corresponding to the critical regime where scale-free avalanches are created. For higher $\Lambda$ (c)-(e), one observes an increase in the number of breathers (multibreather regime) and changes in the stability of the breathers. For strong nonlinearity, for e.g. (e) $\Lambda=16$, the number of breathers is of the order of $M$. The left figure shows a zoom-in view of the profile of a breather in (c) centered at site 29 at time $\tau \approx 600 \times 30$.

The most interesting dynamics emerges for interatomic interaction strengths in a range of criticality

$$
\Lambda_{L}<\Lambda<\Lambda_{U}
$$

where we find multibreather states with a scale-free distribution of single site norms $N_{n}$. The inset of Figure 3.6 shows the distribution $\mathcal{P}\left(x=N_{n}\right)$ for two different system sizes. It displays an inverse power law

$$
\mathcal{P}\left(x=N_{n}\right) \sim x^{-\beta},
$$

with a value $\beta=1.9 \pm 0.05$ given by a best least square fit. The cutoff for small $N_{n}$ seen in Fig. 3.6 can be shifted to arbitrarily small values for larger OLs. In our numerics with system sizes up to $M=4096$ we found the upper and lower bounds $\Lambda_{U} \gtrsim 2$ and $\Lambda_{L} \lesssim 0.5$, with strong indication that in the limit $M \rightarrow \infty$ the lower bound approaches $\Lambda_{b}$. (The role of the critical interaction strength $\Lambda_{b}$ is 
investigated in the Sec. 3.5.) This power law behavior is a signature of the existence of self-organized critical states [100]. In contrast, for $\Lambda<\Lambda_{L}$ we find exponential distributions of the site-norms.

We will see in the following that the size $J$ of the observed avalanches is proportional to the site-norms $N_{n}$ of the critical states. Therefore, one expects that $\mathcal{P}_{N_{n}}(x)$ and $\mathcal{P}(J)$ follow the same asymptotic distribution. Indeed, the best least square fit to our numerical data gives exponents

$$
\alpha \approx 1.86 \approx \beta .
$$

For smaller values of $\Lambda$ the distribution $\mathcal{P}(J)$ shows clear deviations from the power law. For increasing $M$, however, these deviations become smaller as shown in Fig. 3.6 for $\Lambda=0.5$, indicating $\Lambda_{L} \rightarrow \Lambda_{b}$ in the thermodynamic limit $M \rightarrow \infty$.

In Fig. 3.7 (c-e) one can see that for the case of $\Lambda \geq 1.0$, the two outer-most breathers act as barriers which trap the atoms in the bulk of the lattice, preventing them from leaking out towards the absorbing boundaries and slowing down the relaxation process. Thus, DBs are observed to act as dynamical barriers, insulating the leaking boundaries from the central core. It is clear that the appearance of a power law distribution in Eq. (3.13) is associated with the existence of DBs.

\subsection{Order Parameter}

Since we are interested in the effects of DBs on the relaxation process of the DNLS (Eq. 3.5), we introduce a localization parameter $\mathcal{P} \mathcal{R}$ which is a measure of the relative number of sites that are still occupied by the remaining atoms in the leaking OL. It is defined as

$$
\mathcal{P} \mathcal{R}(\tau)=\left\langle\frac{[N(\tau)]^{2}}{M \sum_{n}\left|\psi_{n}(\tau)\right|^{4}}\right\rangle
$$

where $\langle\cdots\rangle$ indicates an average over different initial conditions. For $\gamma=0$, Eq. 3.17 gives the standard participation ratio. Accordingly, the more evenly the atoms spread over the lattice, the closer $\mathcal{P} \mathcal{R}$ is to a constant of $\mathcal{O}(1)$ while a concentration of the whole atomic density in a single site yields $\mathcal{P} \mathcal{R}=1 / M$. The $\mathcal{P} \mathcal{R}$ approaches two limiting values that can be calculated analytically (see Appendix A]: (a) For $U=\Lambda=0$ (linear regime), the norms $N_{n}$ are exponentially distributed, leading to $\mathcal{P} \mathcal{R}=1 / 2$. The case where $\mathcal{P} \mathcal{R}=1 / 2$ corresponds to the situation where there is no DB. (b) For $\Lambda \rightarrow \infty$ (strong nonlinear regime), the wells are uncoupled and the norms uniformly distributed leading to $\mathcal{P} \mathcal{R}=5 / 9$. This is due to the formation of discrete breathers, where the number of DBs is of the order of the total number of lattice sites $M$ (multibreather regime). For $\gamma=0$, the transition between these limiting cases is smooth (Fig. 3.8).

In the open system $(\gamma>0)$ the quantity $\mathcal{P} \mathcal{R}=\mathcal{P} \mathcal{R}(t)$ is of course time dependent. After an initial drop, however, it rapidly approaches a constant value $\mathcal{P} \mathcal{R}_{S}$ indicating a quasi-steady state (Fig. 3.9). In the following we study $\mathcal{P} \mathcal{R}_{S}$ as a function of the interaction strength $\Lambda$. Instead of a smooth transition between the two extremes, as in the closed system, we observe a sharp drop of $\mathcal{P} \mathcal{R}_{S}$ at a 


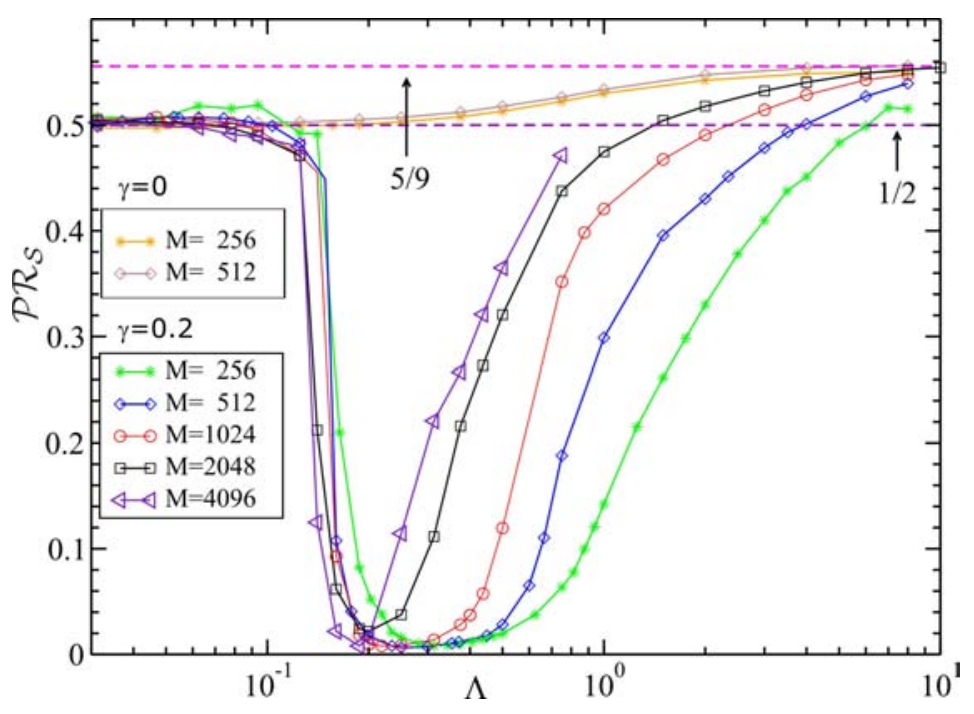

Figure 3.8: The localization parameter $\mathcal{P} \mathcal{R}_{S}$ vs. $\Lambda \equiv \lambda N(\tau=0) / M$ is shown. For the closed system a smooth transition between the limits $1 / 2$ and $5 / 9$, which are given analytically, takes place. However, for the open system a sharp drop in $\mathcal{P} \mathcal{R}_{S}$ is observed, indicating a phase transition (see text).

critical interaction strength of $\Lambda_{b} \approx 0.15$ as shown in Fig. 3.8, resembling a phase transition. Our numerics indicate that this transition indeed becomes a step function in the limit $M \rightarrow \infty$. At $\Lambda_{b}$ the order parameter $\mathcal{P} \mathcal{R}_{S}$ drops down to its lowest possible value $(1 / M)$ corresponding to a single occupied site, i.e. the final state consists of one single DB. We remark that for $\Lambda<\Lambda_{b}$, the atomic population $N(\tau)$ decays smoothly to zero, following the same qualitative behavior as for the $\Lambda=0$.

As we can see from Fig. 3.8, the transition between the linear regime and the case where one DB is created becomes sharper in the thermodynamic limit. This indicates the existence of a phase transition. We have confirmed that the above behavior of the $\mathcal{P} \mathcal{R}$ remains qualitatively the same for various values of $\gamma$ ranging from 0.01 to 1 . For $\Lambda \gg 1$, we recover the strong nonlinearity limit where many breathers are found. However, we do not investigate the nature of the transition (e.g. if a similar 'sharp' transition takes place) in the strong nonlinear limit.

\subsubsection{Nature of the Phase Transition}

To understand the nature of the transition at $\Lambda_{b}$ it is important to realize that if a breather solution exists for some value of $\Lambda$, it exists for all $\Lambda^{\prime} s>0$ (for large enough $M$ ). This can easily be seen by noting that a DB is not directly coupled to the leaking edges, thus we can assume $\gamma=0$ and then appropriately scale Eq. 3.5 . Therefore breather solutions in particular do exist for $\Lambda<\Lambda_{b}$ as well. For every nonlinearity $\Lambda$, however, there exists a lower bound for the norm carried by the DBs (that is well approximated by $\frac{1}{2 M \Lambda}$ ). For the dynamics to end in a single breather state the intermediate thermalized state therefore has to provide a fluctuation large enough to create this breather, and at the same time all other fluctuations have to be small enough not to destabilize the breather again. While a full (analytical) 

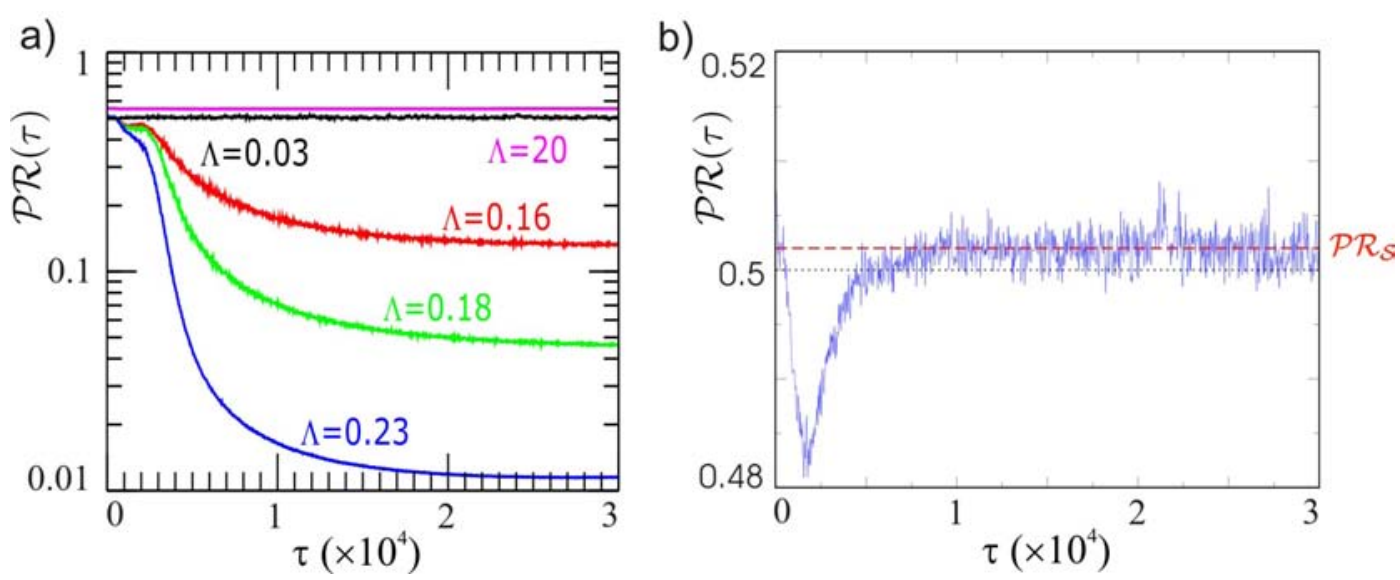

Figure 3.9: (a) For several values of $\Lambda$, the participation ratio $\mathcal{P} \mathcal{R}(t)$ is shown, which converges to a steady state value $\mathcal{P} \mathcal{R}_{S}$. (b) Time evolution of the participation ratio for an open system with $M=2048, \Lambda=0.06$ and dissipation rate $\gamma=0.2$. After an initial drop from 0.5 to approx. 0.48 , the curve fluctuates slightly around a steady state value $P R_{S}$, which is stable for very long times (as long as our simulations ran). The size of the initial drop is supposed to depend on the dissipation rate $\gamma$. In the left figure the initial drop exists as well, but cannot be resolved due to the large scale of the ordinate.

understanding of this process is subject of present research [77, 101] and work in progress [87], our numerical evidence on the existence and nature of this phase transition is very clear.

\subsection{An Avalanche Event}

Let us study in more detail the dynamics that lead to the creation of an avalanche in the critical range of $\Lambda_{L}<\Lambda<\Lambda_{U}$ (where a power law distribution of avalanches is seen in the flux of atoms out of the optical trap). One such event is depicted in Fig. 3.10, A moving breather (MB), coming from the bulk of the lattice, collides with the outermost stationary DB. Note that although MBs can survive for very long time, strictly speaking they are not stationary solutions of the DNLS. As a result of the collision, the DB is shifted inwards by one site while at the same time a fraction of the atomic density of the MB is transmitted through the DB. Eventually this particle density will reach the leaking edge of the OL and decay in a form of an avalanche. Other lattice excitations (e.g. thermal noise) could as well take the role of acting as perturbation to the DB.

To investigate theoretically the collision process that involves the $\mathrm{DB}$, we will make use of a general concept called the local ansatz [49, 102]. The DB solutions in the DNLS are very well localized and the most basic and important breathers occupy only three sites. Within the local ansatz thus only the central site and the two neighboring sites of a DB are considered, allowing us to turn a high dimensional dynamical problem involving $M$ sites, into a reduced problem with three degrees of freedom (non-linear trimer). A detailed analysis of the reduced problem [49, 102, shows that (i) the discrete breather corresponds to a trajectory in the phase space of the full system which is practically embedded on a two-dimensional torus manifold, 


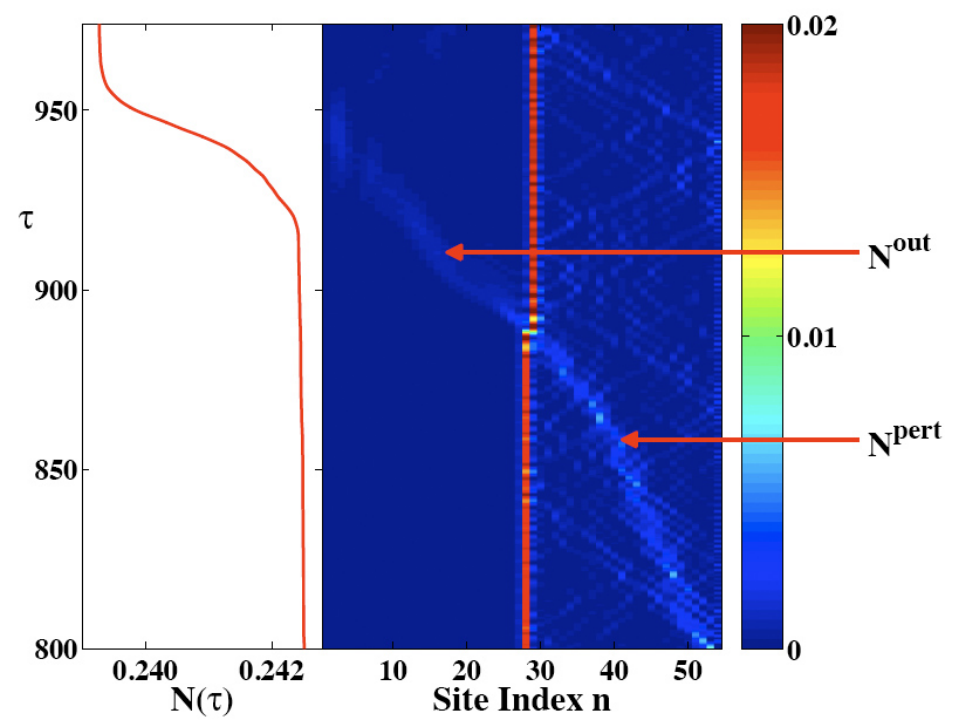

Figure 3.10: Snapshot of an avalanche event. On the left subpanel, we are plotting $\tau$ vs $N(\tau)$ whereas on the right we are reporting a representative collision event between the outermost stationary $\mathrm{DB}$ and a moving $\mathrm{DB}$ (the color indicates the atomic density $N_{n}(\tau)$ ). The moving breather of atomic density $N^{\text {pert }}$ enters the monitored region from the right and collides with the stationary breather. During the collision, the stationary breather gets destabilized and is shifted inwards while part of the moving DB 'tunnels' through the stationary breather and travels towards the edge of the lattice. The arrival of the transmitted density at the edge is registered in the atomic population $N(\tau)$ as an avalanche event (see left subpanel). Note that for illustration, a representative avalanche event in $N(\tau)$ is encircled in Fig. 3.4 .

thus being quasiperiodic in time; (ii) the DB can be reproduced within a reduced $(M=3)$ system, called the nonlinear trimer.

\subsection{Origin of the Scale-free Avalanches}

Equipped with an understanding of an avalanche event, we now develop a physical understanding on the origin of a power law distribution of the jumps through an analysis of phase space structure of the reduced system: the nonlinear trimer [28].

\subsubsection{Poincaré Section of the Trimer}

In Fig. 3.11 we show a representative Poincaré section of the reduced system for interaction strength $\Lambda \approx 1$. The phase space consists of two components: islands of regular motion (tori) embedded in a chaotic sea. Trajectories inside the islands correspond to DBs, provided that their frequency is outside the linear spectrum. In contrast, chaotic trajectories have continuous Fourier spectra, parts of which overlap with the linear spectrum of the infinite lattice [49]. Note that a representative Poincaré section of the nonlinear trimer including disorder (i.e. the on-site chemical potential $\mu_{n}$ is not constant in Eq. 3.4) exhibits as well a mixed phase space [103]. 


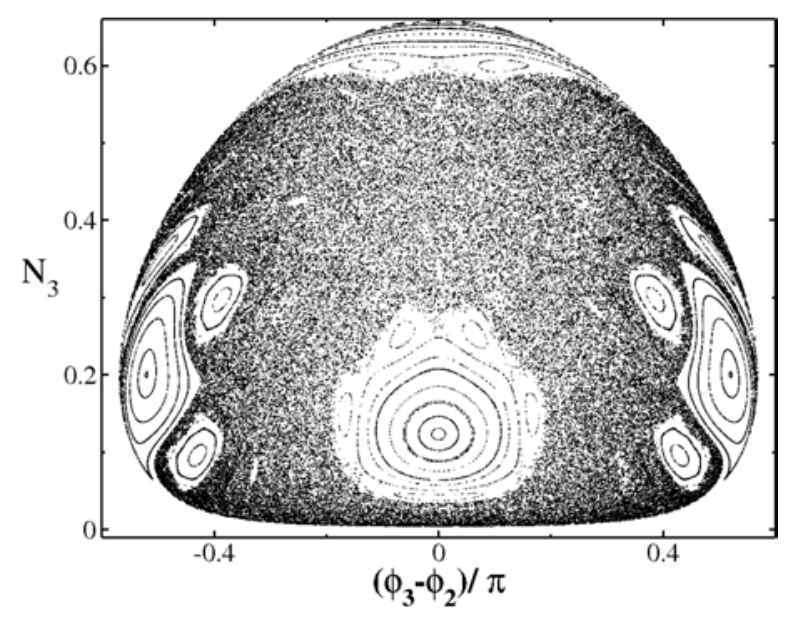

Figure 3.11: A Poincaré section of the phase space of the nonlinear trimer at $\Lambda \approx 1.0$. Shown is $N_{2}$ vs $\left(\phi_{3}-\phi_{2}\right) / \pi$ where $\phi$ 's are the angles in Eq. 3.2. The Poincaré section at $\Lambda \approx 1.0$ corresponds to the plane $\phi_{1}=\phi_{3}$ and $\dot{\phi}_{1}>\dot{\phi}_{2}$ of the energy surface. It clearly shows a hierarchical mixed phase space structure with islands of regular motion (tori) embedded in a sea of chaotic trajectories.

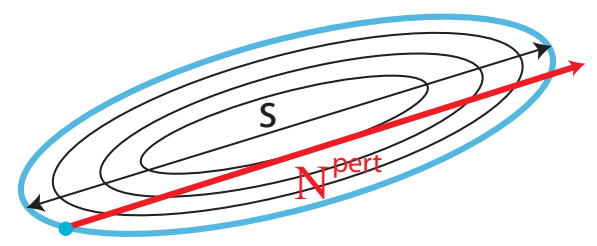

Figure 3.12: Illustration of the arguments leading to Eq. 3.18. The figure shows an island in a background of chaotic sea. Black ellipses correspond to regular orbits in an island, where $s$ is the maximum diameter of the island. The blue ellipse is an example of a regular trajectory of a particle on the island, which corresponds to the case of a DB in our system. The destabilization of a stationary DB by a perturbation (e.g. a thermal fluctuation or a moving breather) with density $N^{\text {pert }} \equiv N_{1}^{\text {pert }}$ is possible only if the DB can be pushed out from the regular orbit across the island towards the chaotic sea. The particle's motion then becomes chaotic, allowing for a continuous Fourier spectra and thus for dramatic increase of frequency overlap with the phonon band. 


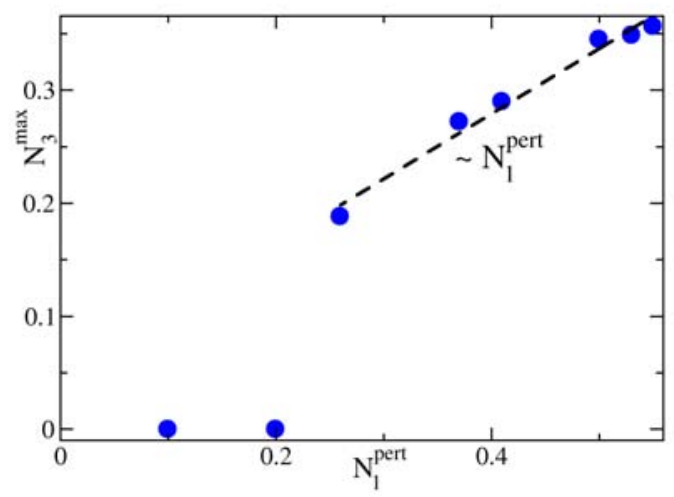

Figure 3.13: A destabilization process of a DB hosted by a closed trimer. We report the outgoing atomic population $N_{3}^{\max } \approx N^{\text {out }}$ (associated with an avalanche event - see Fig. 3.10 measured at site 3 versus the incoming atomic population $N_{1}^{\text {pert }}$ hosted by site 1. We find that atomic population tunnels through the DB only if $N_{1}^{\text {pert }} \gtrsim 0.25$, corresponding to the minimal excitation needed to trigger the destabilization of the DB. Some of the density tunnels through the second site and reaches the third site. We register the maximum density on the third site as $N_{3}^{\max }$ and obtain $N_{3}^{\max } \propto N_{1}^{\text {pert }}$. Analytical expressions for the minimal excitation necessary to destabilize the DB and an upper bound for the transmitted particle density $N_{3}^{\max }$ are calculated in Sec. 3.8 and in [29].

The basic idea to explain the origin of the scale-free avalanches is the following: As long as the DB is stable, it acts as a barrier which prevents atoms from reaching the leaking boundary. Thus, a necessary condition for an avalanche event is the destabilization of the DB. As explained above, this can be caused by a lattice excitation (e.g. a moving breather) with particle density $N_{1}^{\text {pert }}$ greater than a threshold2 ${ }^{2}$. Due to the collision process, the regular or quasiperiodic orbit corresponding to the stationary breather can be pushed out of the island towards the chaotic sea, see illustration in Fig. 3.12. In other words, in order to destabilize a $\mathrm{DB}$, one needs a perturbation that is at least of the order of the linear size $s$ (e.g. the maximum diameter) of the island which represents a DB. At the same time a portion

$$
J \approx N_{3}^{\max } \propto N_{1}^{\text {pert }} \propto s
$$

is transmitted through the DB (Fig. 3.13). Therefore, this destabilization process lets a fraction $N_{3}^{\max } \approx N^{\text {out }}$ of the perturbation tunnel through (see Fig. 3.10) which reaches the leakage at the edge of the lattice, triggering an avalanche. Hence, the task to understand the origin of the power law distribution of jumps $\mathcal{P}(J)$ translates into the study of the distribution of island sizes $\mathcal{P}(s)$.

\footnotetext{
${ }^{2}$ Strictly speaking, this is valid only for a fixed relative phase between the DB and the moving breather as the destabilization holds for the total energy, as explained in Sec. 3.8] and in [29].
} 


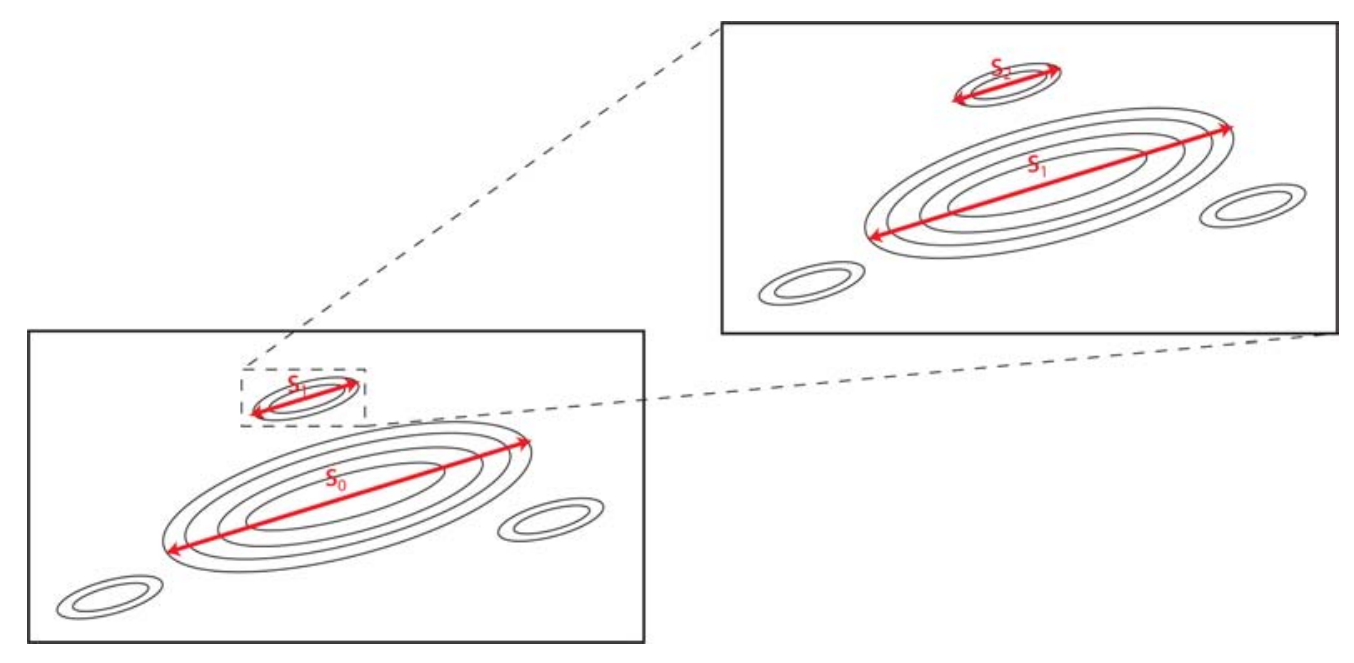

Figure 3.14: Illustration of the simple hierarchical model. This sketch shows the example of $\mathrm{n}=3$. The 0 -th level main island has diameter $s_{0}$ and is surrounded by $n=3$ subislands of size $s_{1}$, in a background of chaotic sea. Due to self-similarity, if we zoom into one of the sub-islands, we would recover the self-similar structure of the islands but now with the main island being in the $k=1$ level with $s_{1}$ diameter, surrounded by another $n=3$ sub-subislands with diameter $s_{2}$.

\subsubsection{A Simple Hierarchical Model}

The above analysis in the frame of the local ansatz, allows us to turn the problem of the analysis of $\mathcal{P}(J)$ to the analysis of the distribution of island sizes $\mathcal{P}(s)$ of the reduced system with $M=3$ in the $\Lambda$-regime where the phase space is mixed. That enables us to determine analytical lower and upper boundaries for $\alpha$ by considering a heuristic model that mimics the hierarchical ('island-over island') structure of a typical mixed phase space in $d$ dimensions. Beforehand, it should be said that we don't aim to consider a simple hierarchical model for high dimensional mixed phase spaces in general, but for dimension $d=2$. However, from a mathematical point of view, our simple heuristic model works in any dimension.

We assume in the heuristic model that at each hierarchy level $k>0$ a main island of linear size $s_{k-1}$ exists with a number $n_{k}$ of sub-islands (see Fig. 3.14 for illustration). As a measure of the linear size $s$ of an island, e.g. the maximum diameter can be taken. At the $k$-th hierarchy level, the fraction of sizes of the main island to sub-island is $f_{k}=\frac{S_{k-1}}{S_{k}}$. Then $s(k)$ reads

$$
s(k)=\frac{s_{0}}{\prod_{i=1}^{k} f_{i}}
$$

and the total number of islands up to level $k$ is given by

$$
p(k)=\prod_{i=1}^{k} n_{i} .
$$

Setting $s_{0}=1$ and making the additional simplifications $f_{k}=f$ and $n_{k}=n$ for each hierarchy level, we obtain

$$
s_{k}=f^{-k}
$$


while the total number of islands $p_{k}$ reads

$$
p_{k}=\prod_{i=1}^{k} n_{i}=n^{k}
$$

Hence, with $k=-\ln s_{k} / \ln f$ from Eq. 3.21 the distribution of island sizes yields

$$
\mathcal{P}(s)=n^{k(s)}=n^{-\ln s / \ln f}=s^{-\alpha},
$$

with exponent $\alpha=\ln n / \ln f$.

Furthermore, analytical bounds for $\alpha$ can be obtained, as the two following limiting cases have to be fulfilled:

1. The total number of islands $N_{\text {island }}(s)$ must diverge for $s \rightarrow 0$ due to the self-similar structure of the phase space, which leads to a lower bound for $\alpha$.

2. The total $d$-dimensional phase space volume $V_{d}(s)$ has to be finite, resulting in an upper bound for $\alpha$.

Thus, for the total number of islands

$$
N_{\text {island }}(s)=\int_{s}^{s_{\max }} p\left(s^{\prime}\right) d s^{\prime}=s^{-\alpha+1}-s_{\max }^{-\alpha+1}
$$

we require that $\lim _{s \rightarrow 0} N_{\text {island }}(s)=\infty$ yielding $\alpha>1$. Secondly, for the total phase space volume $V_{d}(s)$ to be finite, the integral

$$
V_{d}(s)=\int_{s}^{s_{\max }}\left(s^{\prime}\right)^{d} p\left(s^{\prime}\right) d s^{\prime}=s^{-\alpha+1+d}-s_{\max }^{-\alpha+1+d}
$$

needs to converge in the limit $s \rightarrow 0$. This requirement leads to an upper bound $\alpha<1+d$. Hence, the bounds for the power law exponent $\alpha$ read $1<\alpha<1+d$, where $d$ is the dimension of the phase space.

To summarize, for a 2-dimensional mixed phase space our simple hierarchical model yields the analytical restrictions for the power law exponent $\alpha$ of the distribution of island sizes

$$
1<\alpha<3
$$

in excellent agreement with our numerical findings (Eq. 3.16).

\subsubsection{Test with Standard Map}

The power law distribution Eq. 3.23 is surmised to be a generic feature of dynamical systems with a hierarchical mixed phase space in $2 \mathrm{D}$, of which the Poincaré section of the trimer is an example. To get further confidence on our heuristic modeling, we aim to check numerically whether the sizes of islands of a typical Hamiltonian mixed phase space system are really well approximated by a power law distribution. To our knowledge the distribution of island sizes in a mixed phase space has not 


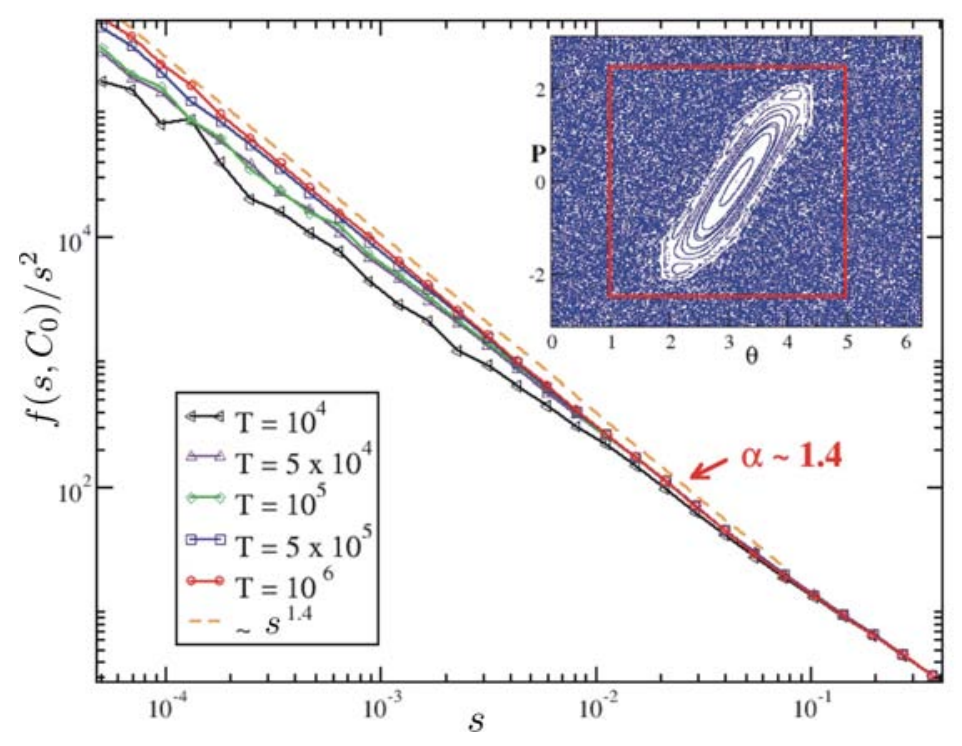

Figure 3.15: Numerical estimation of the distribution of island sizes in a mixed phase space of the standard map for kicking strength $K=3.5$. We plot $f\left(s, C_{0}\right) / s^{2}=\left(p_{\text {in, out }}(s)-\right.$ $\left.C_{0}\right) / s^{2}$, which is a measure of the circumference of the islands vs. their width $s$. Clearly a power law distribution over more than three orders of magnitude is found, with exponent $\alpha=1.4$. The red rectangle in the inset marks the mixed phase space region under investigation (see Fig. 2.3p for consecutive enlargements).

yet been analyzed in literature, which at first came as a surprise. However, when considering the complexity and the hierarchical structure of a typical mixed phase space - see Fig. 2.3b for consecutive enlargements of a typical mixed phase space - it becomes clear, that calculating the distribution of island sizes is a challenge of its own, as for convincing evidence of the existence of a power law distribution, several orders of magnitudes of island sizes have to be resolved.

We will numerically approximate the distribution of island sizes in a paradigmatic model of mixed phase space dynamics: the standard map (see Sec. 2.2.1 for an introduction). We used kicking strength $K=3.5$ for our analysis, hence, for illustration, we chose the same value of $K$ in Fig. 2.3 b. The idea for the numerical approach will be sketched here, while the details are given in appendix $B$.

We consider a region $\mathcal{R}$ (see inset of Fig. 3.15) in the mixed phase space of the standard map containing an island (with its daughter islands) originating from a period 1 resonance ${ }^{3}$. We start two trajectories at random initial conditions $\mathbf{r}$ and $\mathbf{r}+\mathbf{s}$ within $\mathcal{R}$ separated by a distance $s=|\mathbf{s}|$. We follow the trajectories up to time $T$ and proclaim a trajectory to be inside an island if it has not left the region $\mathcal{R}$ after a time $T$ (which will become more and more accurate with increasing $T$ ). The quantity we are going to study is $p_{i n, o u t}(s)$, which is the probability that the first trajectory is inside an island and the second is not. This probes the circumferences of the islands with less and less error (as the size of an island is overestimated with this method) with increasing $T$.

Assuming a power law distribution of island sizes with exponent $\alpha$, we find that $p_{\text {in,out }}(s)$ can be written as (see Appendix B)

\footnotetext{
${ }^{3}$ For a period $\mathrm{N}$ resonance, we could do the same with the $\mathrm{N}$-th iteration of the map.
} 


$$
p_{\text {in,out }}(s)=C_{0}+C_{1} s^{2-\alpha}
$$

with constants $C_{0}$ and $C_{1}$. The constant $C_{0}$ is estimated by

$$
\sigma\left(C_{0}\right)=\min _{C \in I}(\sigma(C))
$$

where $\sigma(C)$ is the standard deviation resulting from a linear fit on a double logarithmic scale of the function

$$
f(s, C)=p_{\text {in,out }}(s)-C,
$$

and $I \subset \mathbb{R}$ is an appropriate interval. We checked that $C_{0}$ changes smoothly with the iteration time $T$ of the standard map.

Figure 3.15 shows the numerical results for $p_{\text {in,out }}(s)$ for different maximal iteration times $T$. As $T$ increases, the curves fit better and better the form of Eq. 3.27. Thus, for the standard map with $K=3.5$, we have estimated a power law distribution of island sizes with an exponent $\alpha=1.4$.

In conclusion, our approximation to the distribution of island sizes clearly exhibits a power-law distribution over more than three orders of magnitude thus confirming the validity of Eq. 3.23. Hence, our results provide numerical evidence that the power law scaling is a generic feature of a typical mixed phase space.

\subsection{Nonlinear Trimer}

The destabilization of a DB due to a collision with a lattice excitation accompanied by a shift of the DB (typically by one site) towards the perturbation which we saw in Fig. 3.10, has been observed in the past in various discrete nonlinear systems [28, 58, 61, 86, 104. Clearly, the stability of DBs with respect to perturbations [61, 75, 105, 106] plays an important role during the collision process. We saw numerically, that beyond a certain threshold the DB can get destabilized while a fraction of the (atomic) density of the lattice excitation tunnels through the DB (Fig. 3.13).

In this Section, let us consider a discrete breather (DB) centered in the middle of a nonlinear trimer. We will analytically calculate the total energy threshold of the nonlinear trimer in order to enable a perturbation at site 1 to trigger the destabilization of a DB centered at site 2 [29]. Furthermore, we will determine an upper boundary for the maximum (atomic) density that is transmitted through the DB in the course of the collision. 


\subsubsection{Equations of Motion}

Our starting point are the equations of motion of the nonlinear trimer. The DNLS with $M=3$ lattice sites reads (see Eq. 3.4)

$$
\begin{aligned}
i \partial_{t} \psi_{1} & =\lambda\left|\psi_{1}\right|^{2} \psi_{1}-\frac{1}{2} \psi_{2} \\
i \partial_{t} \psi_{2} & =\lambda\left|\psi_{2}\right|^{2} \psi_{2}-\frac{1}{2}\left(\psi_{1}+\psi_{3}\right) \\
i \partial_{t} \psi_{3} & =\lambda\left|\psi_{3}\right|^{2} \psi_{3}-\frac{1}{2} \psi_{2},
\end{aligned}
$$

where the wave functions are normalized such that the total atomic population reads $N=\sum_{n=1}^{M}\left|\psi_{n}\right|^{2}=1$. The normalization condition corresponds to Eq. 3.12 . however, here we are dealing with a closed system where the total norm $N$ is conserved. In order to find exact DB solutions in Eq. 3.30 for the symmetric case $\psi_{1}=\psi_{3}$, we make the ansatz

$$
\psi_{n}(t)=A_{n} e^{i w t}
$$

with Amplitude $A_{n}$ and frequency $w$ (the latter which is the same for all three sites). This ansatz together with the conservation of norm yields the set of equations

$$
\begin{aligned}
-w A_{1} & =\lambda A_{1}^{3}-\frac{1}{2} A_{2} \\
-w A_{2} & =\lambda A_{2}^{3}-A_{1} \\
1 & =2 A_{1}^{2}+A_{2}^{2} .
\end{aligned}
$$

The equations below will be written in terms of the nonlinearity $\lambda=2 U / T$. Note that the equations can be easily rewritten in terms of the effective interatomic interaction per site $\Lambda=\lambda / 3$ (see Eq. 3.6), e.g. to apply the results to extended lattices.

\subsubsection{Asymptotic Solutions}

What are the symmetric $\left(\psi_{1}=\psi_{3}\right)$ DB solutions for a given nonlinearity $\lambda$ ? First, we calculate the norm $N_{2}=A_{2}^{2}$ at the central site from Eqs. 3.32 which yields

$$
\lambda\left(N_{2}\right)=\frac{\sqrt{2}\left(2 N_{2}-1\right)}{\sqrt{N_{2}\left(1-N_{2}\right)}\left(3 N_{2}-1\right)} .
$$

For $\lambda<5.04$ only one symmetric breather solution exists, while for $\lambda>5.04$ there are several solutions (see Fig. 3.16). To gain further insight into the nature of the symmetric breather solutions in the trimer, we will revisit Eqs. 3.32, which we turn into a quartic equation

$$
x^{4}+\frac{\lambda}{\sqrt{2}} x^{3}-\sqrt{2} \lambda x-1=0
$$

where

$$
A_{2}=\cos (\arctan (x))=\frac{\operatorname{sig}(x)}{\sqrt{1+x^{2}}} .
$$




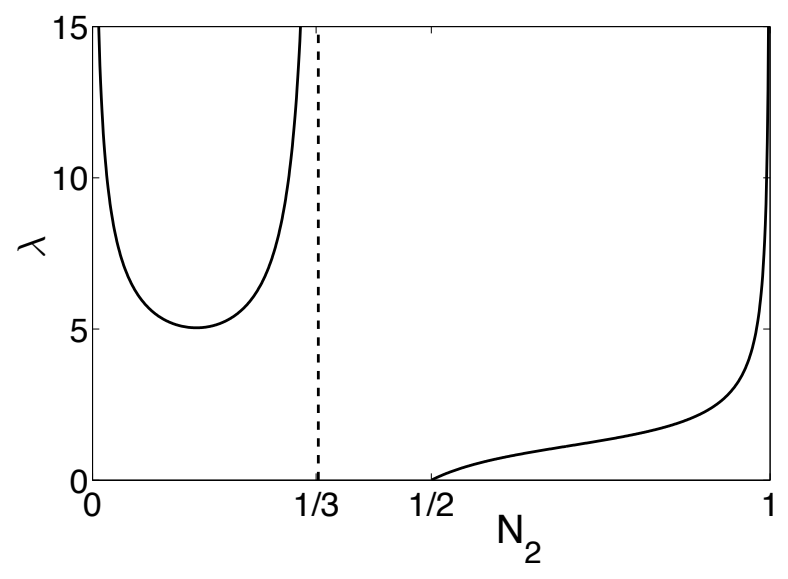

Figure 3.16: DB solutions for the symmetric case $\psi_{1}=\psi_{3}$. The repulsive case is treated explicitly $(\lambda>0)$, however, Eq. 3.33 and the equations below hold as well for the attractive case (as explained in Sec. 3.2). We see that for nonlinearity $\lambda>5.04$ three symmetric breather solutions exist with $\left|\psi_{2}\right|^{2}>0$, where the solution for large norm at the central site is termed a bright breather, see Eq. 3.37 .

Expansion of the exact real solutions of Eq. 3.34 in $\lambda$ for the limiting case $\lambda \rightarrow 0$ yields

$$
\begin{aligned}
& x_{1}=1+\frac{\lambda}{4 \sqrt{2}}-\frac{5}{64} \lambda^{2}+\mathcal{O}\left(\lambda^{3}\right) \\
& x_{2}=-1+\frac{\lambda}{4 \sqrt{2}}+\frac{5}{64} \lambda^{2}+\mathcal{O}\left(\lambda^{3}\right) .
\end{aligned}
$$

At $\lambda=0$ the solution $\vec{\psi}=\left(\psi_{1}, \psi_{2}, \psi_{3}\right)$ reads $\vec{\psi}_{(x 1, x 2)}^{0}=(1 / 2, \pm 1 / \sqrt{2}, 1 / 2)$. Expansion around the limit $\lambda \rightarrow \infty$ leads to four real solutions

$$
\begin{aligned}
& x_{1}=-\frac{1}{\sqrt{2}} \frac{1}{\lambda}-\frac{1}{4 \sqrt{2}} \frac{1}{\lambda^{3}}+\mathcal{O}\left(\lambda^{-5}\right) \\
& x_{2}=-\frac{1}{\sqrt{2}} \lambda+2 \sqrt{2} \frac{1}{\lambda}+14 \sqrt{2} \frac{1}{\lambda^{3}}+\mathcal{O}\left(\lambda^{-5}\right) \\
& x_{3}=-\sqrt{2}-\frac{3}{2 \sqrt{2}} \frac{1}{\lambda}-\frac{69}{16 \sqrt{2}} \frac{1}{\lambda^{2}}+\mathcal{O}\left(\lambda^{-3}\right) \\
& x_{4}=\sqrt{2}-\frac{3}{2 \sqrt{2}} \frac{1}{\lambda}+\frac{69}{16 \sqrt{2}} \frac{1}{\lambda^{2}}+\mathcal{O}\left(\lambda^{-3}\right) .
\end{aligned}
$$

For infinite $\lambda$ the solutions read

$$
\begin{aligned}
\vec{\psi}_{(x 1)}^{\infty} & =(0,1,0) \\
\vec{\psi}_{(x 2)}^{\infty} & =(\sqrt{1 / 2}, 0, \sqrt{1 / 2}) \\
\vec{\psi}_{(x 3, x 4)}^{\infty} & =(1 / \sqrt{3}, \mp 1 / \sqrt{3}, 1 / \sqrt{3}),
\end{aligned}
$$

where the solution $\vec{\psi}_{(x 1)}$ is called a bright breather, $\vec{\psi}_{(x 2)}$ is called a dark breather (due to lack of norm at the central site) and $\vec{\psi}_{(x 3, x 4)}$ are phase-wise and antiphasewise time-periodic localized solutions. In the following, the fixpoint corresponding 

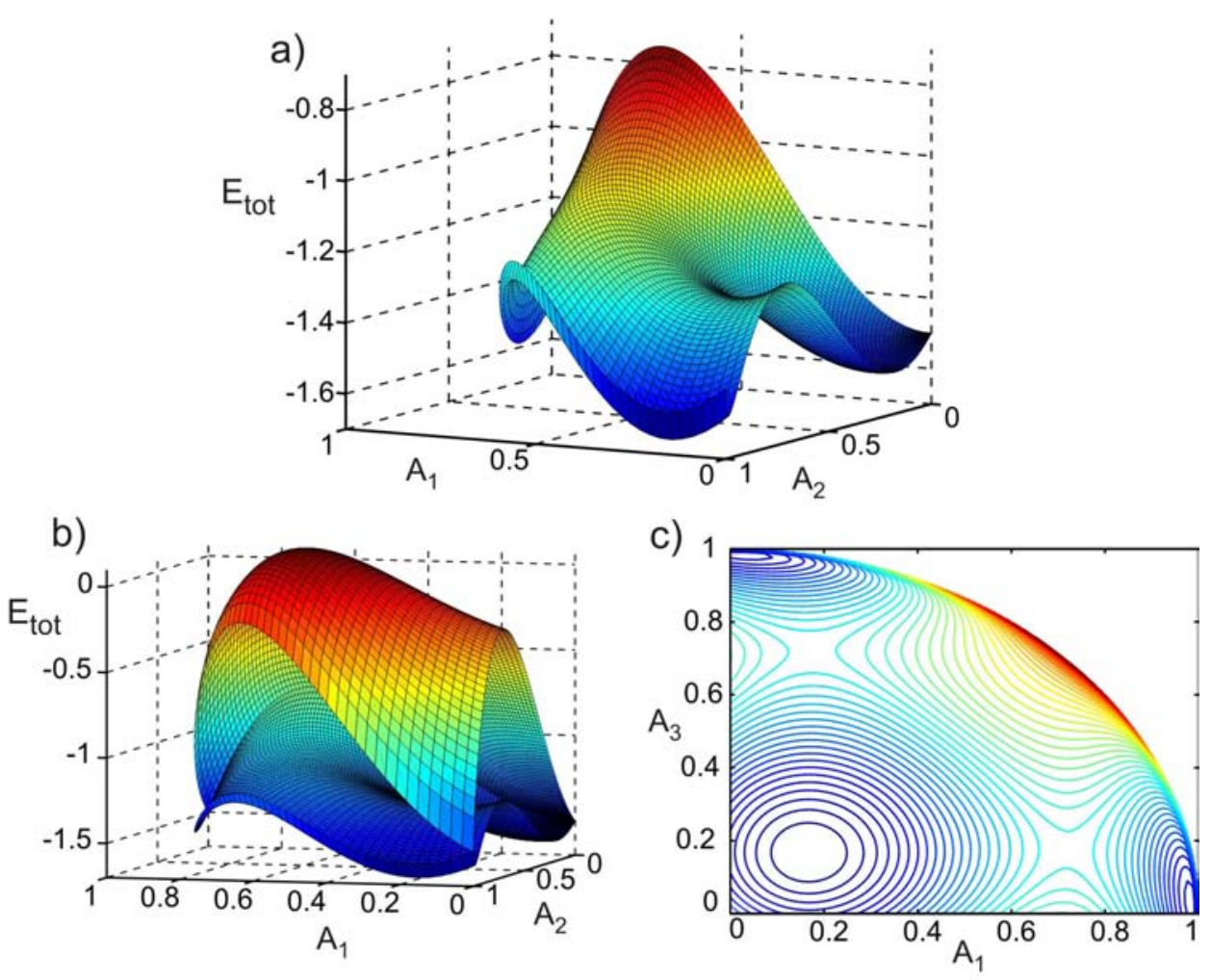

Figure 3.17: (a) PN energy landscape (lower part) (b) PN shell and (c) Contour plot of (a). The three minima of the lower bound to the dynamics correspond to the bright breather solutions at the three sites. All plots are shown for nonlinearity $\lambda=3$.

to the bright breather $\vec{\psi}_{(x 1)}=\left(A_{1}^{f i x}, A_{2}^{f i x}, A_{3}^{f i x}\right)$ will be analyzed with respect to perturbations.

\subsubsection{Peierls-Nabarro Energy Landscape}

We define the Peierls-Nabarro energy landscape as follows: It is obtained for a given configuration of amplitudes $A_{n}$, by extremizing $H$ with respect to the phase differences $\delta \phi_{i j}=\phi_{i}-\phi_{j}$. where $\psi_{n}=A_{n} \exp \left(i \phi_{n}\right)$ :

$$
H_{p n}^{l}=\min _{\delta \phi_{i j}}(-H) \quad ; \quad H_{p n}^{u}=\max _{\delta \phi_{i j}}(-H),
$$

where $H_{p n}^{l}$ and $H_{p n}^{u}$ are the lower and upper part of the PN landscape. The phase differences extremizing the Hamiltonian

$$
H=\frac{\lambda}{2}\left(A_{1}^{4}+A_{2}^{4}+A_{3}^{4}\right)-\left(A_{1} A_{2} \cos \left(\phi_{1}-\phi_{2}\right)+A_{2} A_{3} \cos \left(\phi_{2}-\phi_{3}\right)\right)
$$

are $\delta \phi_{12}=\delta \phi_{23}=(0, \pi)$. The PN energy landscape reads

$$
H_{p n}^{l, u}=-\frac{\lambda}{2}\left(A_{1}^{4}+A_{2}^{4}+A_{3}^{4}\right) \mp\left(A_{1}+A_{3}\right) A_{2}
$$

which is, due to conservation of norm $\left(A_{2}^{2}=1-A_{1}^{2}-A_{3}^{2}\right)$, a function of two amplitudes. In Fig. 3.17 the PN landscape is visualized for $\lambda=3$. It exhibits 

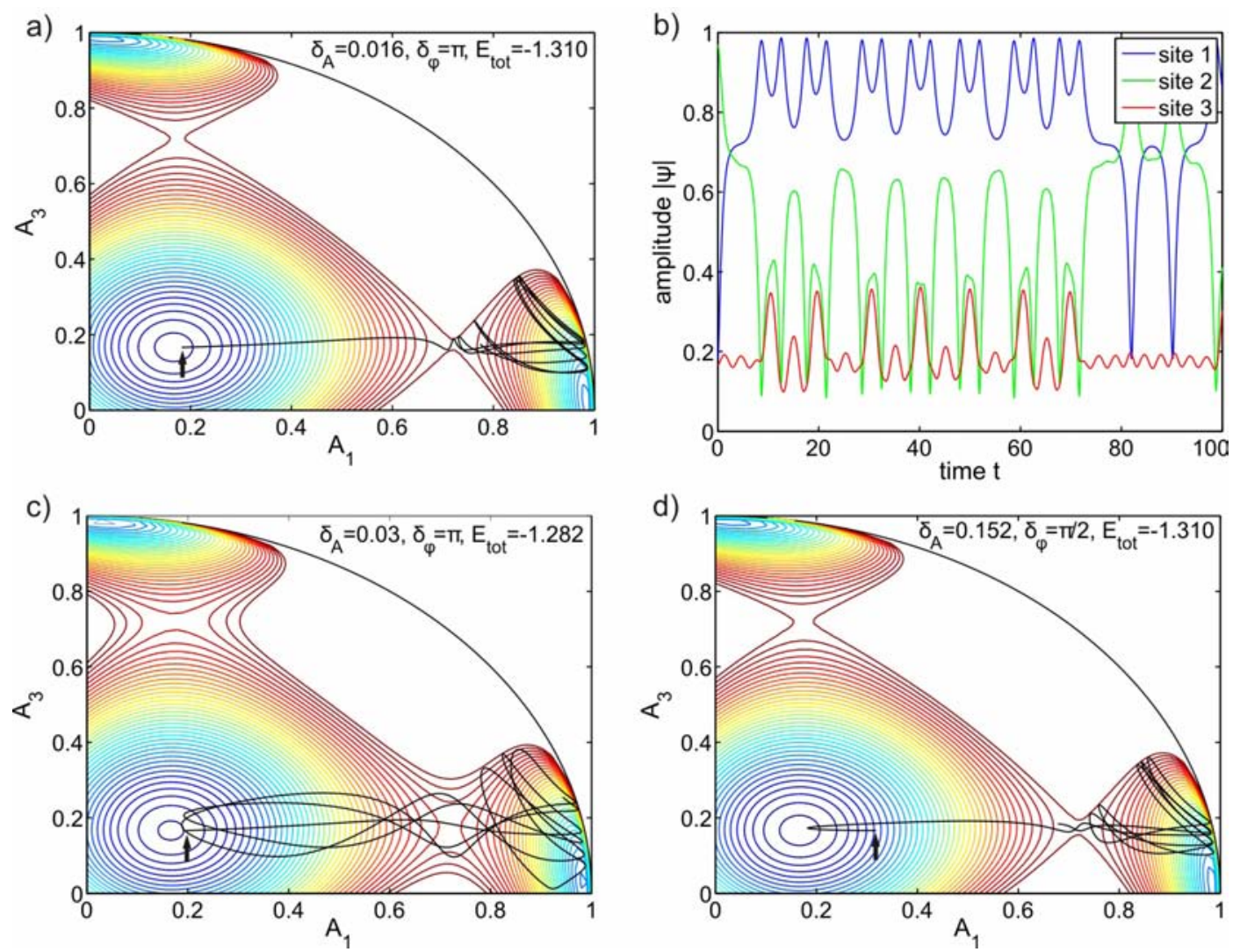

Figure 3.18: Dynamics on the PN landscape. (a, c, d) A contour plot of the lower PN energy landscape $H_{p n}^{l}$ is shown for total energy $E_{t o t}>E_{t h r s}=-1.311$, i.e. above the rim. A projection of the orbit for times $t=0 \ldots 35$ onto the $A_{1}-A_{3}$ plane is over-plotted (black curve), where the arrows mark the position at time $t=0$. The input values for the initial condition defined by Eq. 3.43 are given in the figures. In all cases the DB is destabilized and the largest part of the norm migrates to site 1 . We see that the PN energy landscape clearly restricts the dynamics in all cases. $(\mathbf{a}, \mathbf{b})$ The dynamics for a total energy just above the rim $\left(E_{t o t}=-1.310\right)$ and corresponding amplitudes $A_{i}(t)$ are shown. (c) Dynamics for larger total energy $E_{t o t}=-1.282$. (d) For the same total energy $E_{t o t}=-1.310$ as is (a) but a different initial phase difference $\delta_{\phi}$ the same qualitative behavior is found.

three minima corresponding to the bright breather solutions which are separated by saddle points (called 'rim' in the following) located for $\lambda \rightarrow \infty$ at $A_{i}=\sqrt{1 / 2}$ for $i=1,3$. The energy threshold $E_{\text {thrs }}$ at the rim (obtained from Eq. 3.40 reads

$$
E_{\text {thrs }}=\frac{\lambda}{4}+\frac{1}{2}+\frac{1}{4 \lambda}+\mathcal{O}\left(\lambda^{-2}\right)
$$

In order to investigate whether and in which way the PN landscape influences the dynamics of a perturbed fixpoint solution and the transfer of norm through the DB to the other side, we will look at two quantities in the $A_{1}-A_{3}$ plane (plotted on top of each other in Fig. $3.18(\mathrm{a}, \mathrm{c}, \mathrm{d}))$ : First, the projection of an orbit onto the $A_{1}-A_{3}$ plane of a perturbed $\mathrm{DB}$ and second, the contour plot of the $\mathrm{PN}$ landscape 


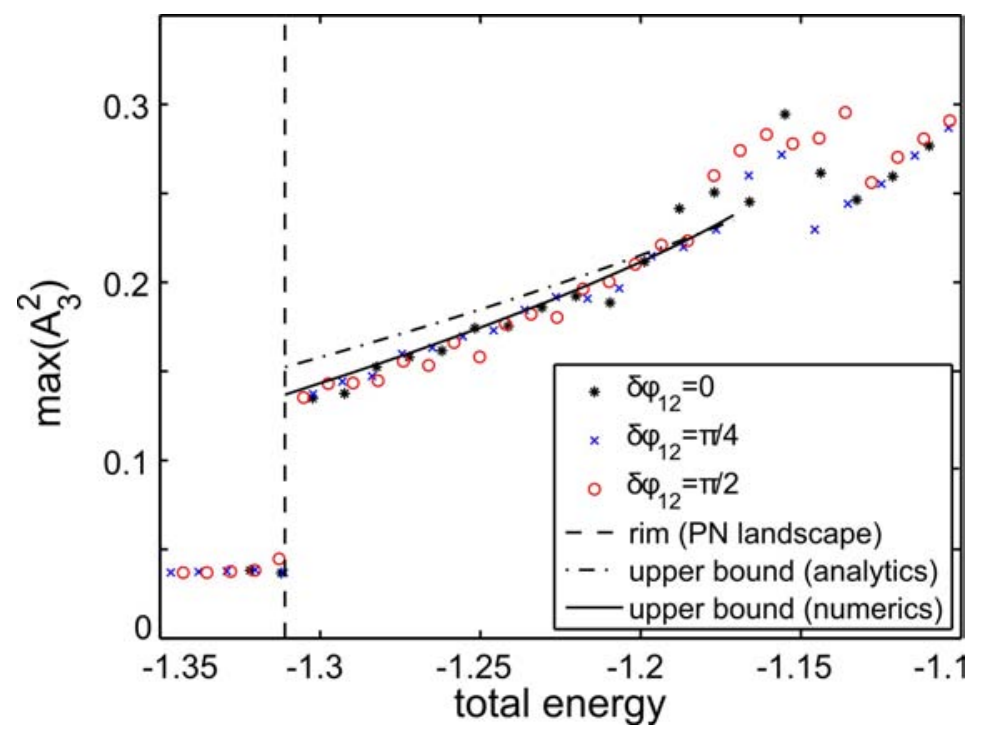

Figure 3.19: Maximum norm $A_{3}^{2}$ that is transferred from site 1 to site 3 while the DB centered at site 2 migrates to site 1 as a function of the total energy $E_{t o t}$. In all cases we set the initial phase difference $\delta \phi_{23}=\pi$ and $\lambda=3$. Note that with $\lambda=3$ (i.e. $\Lambda=1$ ) we reside in the critical regime where scale-free avalanches are found (see Eq. 3.14). The vertical dashed line indicates the destabilization threshold $E_{t h r s}$ (e.g. a threshold for targeted energy transfer, see Sec. 3.9p.

$H_{p n}^{l}$, both at a given total energy $E_{t o t}:^{4}$ We choose the following initial condition:

$$
\vec{\psi}(t=0)=\left(-\left(A_{1}^{f i x}+\delta_{A}\right) e^{i \delta_{\phi}}, A_{2},-A_{3}^{f i x}\right),
$$

where $A_{2}=\left(1-\left|\psi_{1}\right|^{2}-\left|\psi_{3}\right|^{2}\right)^{1 / 2}$ is given by the conservation of norm. With Eq. 3.43 the PN landscape $H_{p n}^{l}$ is determined by the two parameters $\delta_{A}$ and $\delta_{\phi}$. In all cases we set $\lambda=3$, which leads to the destabilization threshold $E_{\text {thrs }}=-1.311$ according to Eq. 3.42 .

Fig. 3.18 shows that the PN energy landscape rigorously restricts the orbits while the rim governs the destabilization process. Note that the larger $E_{t o t}$, the larger is the size of the "bubble" (with the term bubble we denote the accessible region of the PN landscape for $A_{1}>1 / \sqrt{2}$ ). In the limit $\lambda \rightarrow \infty$ the DB in the center of the trimer gets destabilized for $A_{1}>1 / \sqrt{2}$. An upper limit to the norm that is transmitted to site 3 is given by $\max \left(A_{3}\right)_{\mid A_{1}>1 / \sqrt{2}}$, which is the maximum value of $A_{3}$ inside the bubble. As we see from Fig. $3.18 \mathrm{~d}$, the migration of the DB followed by transfer of norm through the barrier is observed as well for other initial phase differences (shown is $\delta \phi_{12}=\pi / 2$ ). Moreover, we have checked that the mechanism itself is widely independent of the choice of parameters, i.e. it is found for a whole range of $\lambda, \delta \phi_{12}$ and $E_{t o t}$.

In Fig. 3.19 , the maximum Norm $A_{3}^{2}$ that is transmitted through the DB as a function of the total energy is shown for three initial phase differences $\delta \phi_{12}=$ $0, \pi A, \pi \not 2$. The dashed vertical line at $E_{t o t}=E_{t h r s}=-1.311$ marks the total energy at the rim and is identified with the destabilization threshold of the DB.

\footnotetext{
${ }^{4}$ The upper PN landscape $H_{p n}^{u}$ may affect the dynamics as well, however, in terms of the destabilization process it is sufficient to focus on the lower part $H_{p n}^{l}$.
} 
The maximum norm at side 3 is closely below the approximated upper bounds determined by the maximum possible value of $A_{3}$ inside the bubble of $H_{p n}^{l}$, where the dashed-dotted curve results from an analytical calculation in the limit for very large $\lambda$ and the solid curve is a numerical calculation. Thus, we found an upper bound to the norm transferred through the DB.

To summarize, the threshold and the tunneling process during the collision of a DB with a lattice excitation (e.g. a moving breather) was described analytically by defining the 2-dimensional Peierls-Nabarro energy landscape. The PN landscape restricts the dynamics of the trimer and the accessible region of the phase space. This restriction of the dynamics becomes very pronounced at the destabilization threshold, which is identified with a rim in the PN landscape.5 The effect is described in a broad range of the nonlinearity $\lambda$ of the system and for any phase difference $\delta \phi_{12}$ between the DB and the MB.

\subsection{Possible Applications}

The investigation of an individual avalanche in Fig. 3.10 triggered our analysis of the collision process of a DB and a moving breather. We observed a threshold for the total energy below which a DB acts as a dynamical barrier (a reflecting 'wall') while above the threshold the MB can destabilize the DB and part of the (atomic) density of the MB tunnels through the DB. This process could lead to several applications.

One possible application refers to atom lasers, in particular the findings may lead to interesting applications for blocking and filtering atom beams. It could be a powerful tool for controlling the transmission of matter waves in interferometry and quantum information processes [106].

In a similar way, our findings can be related to the field of optics, as the DNLS is capable of describing wave motion in nonlinear optical waveguide arrays (where controlled boundary leakage can be achieved with suitable mirrors [2]). Note that the longitudinal space dimension of the waveguides plays the role of the time variable in the DNLS. Discrete breathers in such two-dimensional networks were investigated in the past years both theoretically and experimentally [1,3, 54, 55, 74, 88, 92 ] and can exhibit a rich variety of functional operations such as blocking, routing or logic functions [4, 5] (see Fig. 3.20). Experimental evidence of the destabilization process of the stationary DB (described above) should be observable in nonlinear waveguide arrays and might lead to functional operations such as filtering optical beams.

Another field of conceivable applications refers to the analysis of the destabilization of a DB in the reduced system: The nonlinear trimer. Applications could be possible in terms of a molecular trimer, in particular in biophysics. "If 'discrete breathers' is the answer, what is the question?" This is the title of an article written by G. P. Tsironis [62] giving an overview on how DBs could play a role in biophysics. Let us consider a DB as a bundle of highly (exponentially) localized en-

\footnotetext{
${ }^{5}$ Furthermore, it can be shown [29] that the destabilization threshold during the collision of the two breathers can be linked to the Peierls-Nabarro barrier of a single DB (The Peierls-Nabarro barrier is described in Sec. 2.4 and e.g. in [60, 61]).
} 


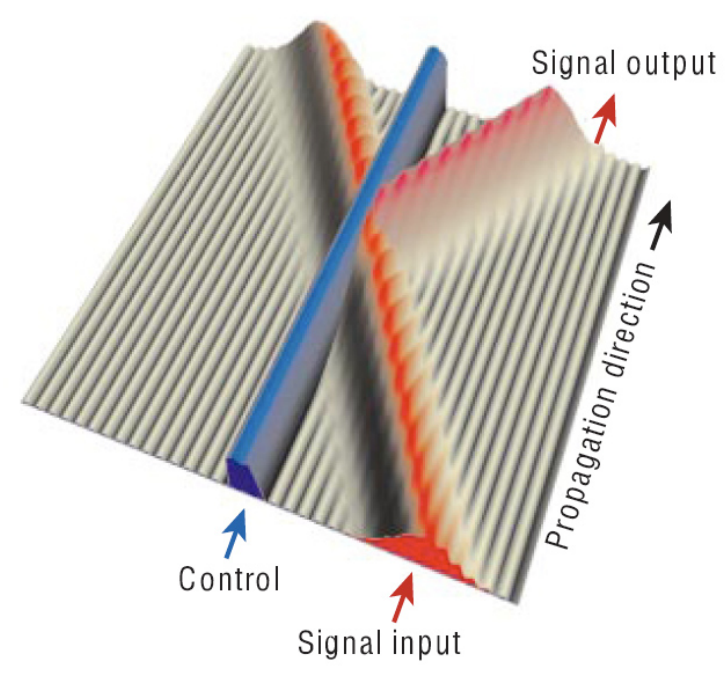

Figure 3.20: Scheme of possible dynamics of discrete (moving) breathers in nonlinear waveguide arrays that shows all-optical routing of on input signal beam to a specific output position [5]. The signal input beam (red) crosses the array with virtually no diffraction. A control beam (blue) at about the second harmonic wavelength is used to produce a third diffractionless output beam.

ergy for a moment, then it is conceivable that a DB could indeed mediate so called targeted energy transfer (TET) [107] - an example for TET could be the transport of energy obtained by the hydrolysis of ATP from a donor site D through a macromolecule (e.g. a motor protein) to an acceptor site A with virtually no dissipation. In the framework of the DNLS, Kopidakis et al. investigated TET between discrete breathers of two weakly coupled random nonlinear systems. These systems could represent two independent molecules, two linked parts of the same folded macromolecule etc. In a real physical or biological system the D-A pair may be interacting with additional degrees of freedom. Under appropriate conditions, irreversible targeting occurs with almost complete energy transfer. Hence, the thresholded transfer of energy through a discrete breather that has been described in this chapter could possibly play a role as well in molecular systems, e.g. in the field of biophysics or biomolecular engineering. However, Tsironis concludes:

"...we see that breathers could in principle act as able energy managers in biomolecules; the real question of course is whether nature is actually using their services."

\subsection{Conclusions}

In conclusion, we have studied the dynamics of BECs in a leaking optical lattice in the framework of the DNLS. In particular, we have observed scale-free avalanches in the decay of the atomic population, and showed that the avalanche events are related to the existence of DBs inside the OL. We provided an explanation of these events using the scenario of a collision process involving a stationary DB and a lattice excitation (e.g. a moving breather) where a threshold of the total energy 
exists, beyond which the DB gets destabilized. The fact that for a certain range of (a rescaled) nonlinearity $\Lambda \sim \mathcal{O}(1)$ a power law distribution $\mathcal{P}(J) \sim J^{-\alpha}$ of avalanches is found, suggests the existence of a phase transition in the system. To this end, we proposed an order parameter $\mathcal{P} \mathcal{R}$ which measures the relative number of sites that are occupied by DBs. We have linked the observed power law distribution of jumps to the hierarchical structure of a mixed phase space shown by the reduced system - the closed nonlinear trimer. Furthermore, a thorough analysis of the PN energy landscape of the closed trimer yields an analytical expression of the minimal excitation necessary to destabilize the DB.

Outlook To better understand the nature of the observed phase transition (at nonlinearity $\Lambda=\Lambda_{b}$ ), it would be of high interest to find an (analytical) expression for $\Lambda_{b}$ as described in Sec. 3.5.1. The transition is related to the formation of DBs in the discrete nonlinear system and is subject of recent research [77, 101] and work in progress [87].

Secondly, it would be very interesting to analyze the nonlinear trimer with dissipation (at the ends) with respect to the observed mechanism of destabilization of a DB followed by a transfer of atoms/energy through the DB. For the closed (i.e. non-dissipative) trimer, the analysis can be found in Sec. 3.8. When dissipation is included in the analysis, a link to experimental systems (molecular trimer) might be possible. A promising approach could be the Holstein model (see e.g. [1] for a detailed description of the Holstein model).

Another system of interest consists of three nonlinear sites (allowing for the destabilization and shift of the DB) embedded in a linear chain (see as well [106]) to enable the investigation of collisions of DBs with lattice excitations and to compare with the results on the stability of DBs in the nonlinear trimer. 


\section{Chapter 4}

\section{Fractal Fluctuations}

\subsection{Mesoscopic Conductance Fluctuations}

While in Chap. 3 the transport properties of bosons in leaking optical lattices are described, here we focus on electronic transport in open mesoscopic systems. In mesoscopic systems conductance fluctuations are a sensitive probe of electron dynamics and chaotic phenomena. A prominent feature of electronic transport in mesoscopic systems is that the conductance as a function of an external parameter, e.g. a gate voltage or a magnetic field, shows reproducible fluctuations caused by quantum interference [14-16]. Typical examples of a chaotic mesoscopic system in which these universal conductance fluctuations were predicted and measured, are quantum billiards (Fig. 4.1) [15]. See Sec. 4.1.1 for an overview.

A prediction from semiclassical theory that inspired a number of both theoretical and experimental works in the fields of mesoscopic systems and quantum chaos was that in chaotic systems with a mixed phase space these fluctuations would result in fractal conductance curves (as described in Sec. 4.1.3) [17, 18]. Such fractal conductance fluctuations (FCF) have since been confirmed in gold nanowires and in mesoscopic semiconductor quantum dots in various experiments [19-23]. In addition, FCF have more recently been predicted to occur in strongly dynamically localized [24] and in diffusive systems [25].

Motivated by puzzling experimental results showing a dependence of the fractal dimension on the coherence length, we studied the classical limit of transport

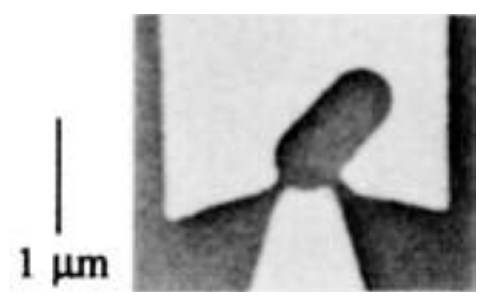

Figure 4.1: Electron micrograph of a ballistic semiconductor quantum dot resembling a stadium billiard with $1 \mu \mathrm{m}$ bar to show the scale. The picture is taken from ref. [15]. Transport measurements by Marcus et. al. exhibit universal conductance fluctuations as a function of an applied perpendicular magnetic field (for moderate fields smaller than 3T) [15]. 
a)

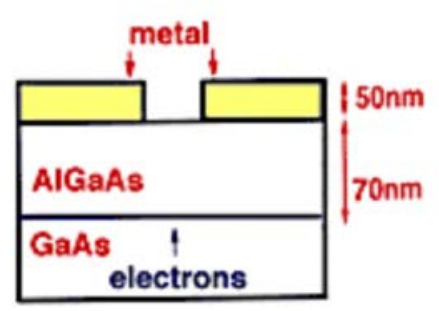

b)

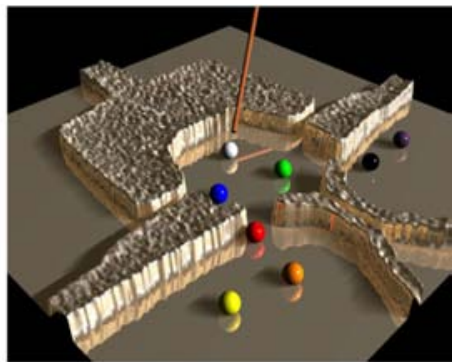

Figure 4.2: (a) Scheme of a 2DEG (figure taken from materialscience.uoregon.edu/taylor/science/taylor_lab.html). (b) Illustration of an electron billiard. The illustration shows some analogy to a macroscopic billiard, however, it does not account for quantum effects.

through quantum dots also finding fractal fluctuations. This chapter will show, that the conductance of purely classical (i.e. incoherent) low-dimensional Hamiltonian systems very fundamentally exhibits fractal fluctuations, as long as transport is at least partially conducted by chaotic dynamics. The fractal dimension is determined analytically and it will be demonstrated, that the fractal dimension is governed by fundamental properties of chaotic dynamics. In addition, we predict that FCF are not only observable in systems with a mixed phase space but in purely chaotic systems 30 . Note that in this chapter, in order to determine the fractal dimension of the graph of a function $T(k)$, we used the variation method (and double checked with the Meakin method), i.e., we calculated $N(\Delta k)=V(\Delta k, T(k)) /(\Delta k)^{2}$, where $N(\Delta k) \propto(\Delta k)^{-D}$ for a fractal curve of dimension $D$, as described in Sec. 2.3 .

\subsubsection{Electron Billiards}

What is a mesoscopic electron billiard? Imagine a two-dimensional electron gas (2DEG) in a semiconductor heterostructure, where electrons can flow freely in a plane, as shown in Fig. 4.2. When a negative voltage is applied to a metallic gate on top of the heterostructure, a depletion area forms inside the 2DEG from which the electrons are repelled. By choice of the geometry of the metallic gates, a quantum dot (we will call it a cavity) can be designed in the plane of the 2DEG. Two or more small channels connect the cavity to outside leads in order to enable conductance measurements. Typically, the size of the mesoscopic cavity is around $1 \mu \mathrm{m}$. If a single particle picture is valid (e.g. the electron correlation can be neglected) and if disorder or impurities are absent, then the transport is ballistic. Furthermore, if the cavity boundaries are clean and steep enough such that the electrons can be considered to reflect specularly from the "walls", the cavity is called a mesoscopic billiard, in analogy to macroscopic billiards. In contrast to macroscopic electronic transport, where Ohm's law is valid, quantum effects come into play, see [108] for an introduction. An important and well understood example is that the conductance through a mesoscopic ballistic cavity forming a chaotic billiard shows reproducible universal fluctuations as an external parameter, e.g. a magnetic field applied perpendicular to the plane of the 2DEG, is varied [15]. 


\subsubsection{Universal Conductance Fluctuations}

Universal conductance fluctuations (UCF), which initially were studied in disordered mesoscopic conductors, are reproducible fluctuations of the conductance (as a function of an external parameter such as the magnetic field or the energy) on the order of $2 e^{2} / h$ and are a quantum effect. The size of the fluctuations is independent of specific system parameters or the average conductance, see Fig. 4.3 for an example. Where do UCF emerge from? In a disordered mesoscopic conductor - which is smaller than the phase coherence length of the charge carriers but large compared to the average impurity spacing - the transmission is the result of the interference of many different, multiply-scattered and complicated paths through the system. As these paths are typically very long compared to the wave length of the charge carriers, the accumulated phase $\theta$ along the paths changes basically randomly when an external parameter such as the energy or the magnetic field is varied. This results in a random interference pattern, i.e. reproducible fluctuations in the conductance of a universal magnitude on the order of $2 e^{2} / h$. For a review see [33] or [109]. The role of disorder in providing a distribution of random phases can as well be taken by chaos. Thus, ballistic mesoscopic cavities like quantum dots in high mobility two-dimensional electron gases that form chaotic billiards show the same universal fluctuations [15, 110, 111]. While for disordered media the shape of the conductor is typically not relevant, it plays an important role for billiards. Assuming chaotic dynamics in the billiard, characterized by an exponential decay of the classical staying probability $P(t) \propto \exp (-\gamma t)$, semiclassical arguments lead to UCF [112]. The classical decay rate is reflected in the autocorrelation function

$$
C(\Delta E)=<g(E) g(E+\Delta E)>_{E}
$$

and leads to a Lorentzian for small $\Delta E$ :

$$
C(\Delta E)=\frac{C(0)}{1+\left(\frac{\Delta E}{\gamma \hbar}\right)^{2}} .
$$

The Lorentzian shape was found experimentally in chaotic billiards [110]. In Fig. 4.4 UCF are shown, which we generated numerically by means of a Lorentzian $C(\Delta E)$. Note that the conductance curve is smooth on small scales.

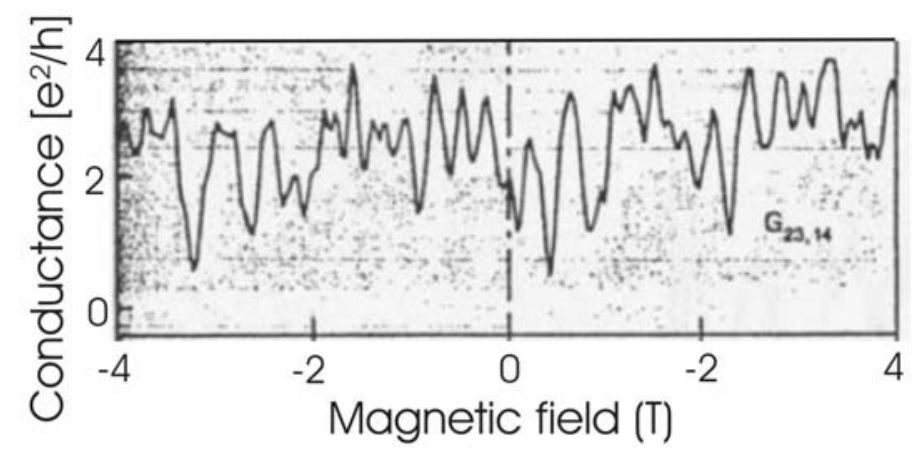

Figure 4.3: Measurement of UCF on a gold nanowire in the late 1980s at temperature $T \approx 0.04 \mathrm{~K}$ (figure adapted from [14]). The fluctuations were reproducible after days of measurement. 


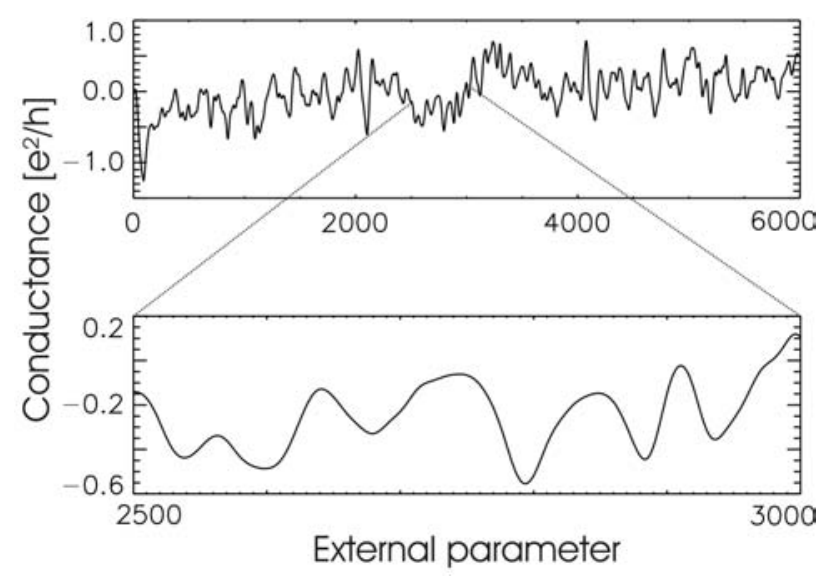

Figure 4.4: UCF, which are generated numerically by means of the autocorrelation function $C(\Delta E)$ from Eq. 4.1 as a function of an external parameter (e.g. the energy or a magnetic field) are shown. The conductance was calculated according to $g(\Delta E)=\mathcal{F}[\sqrt{S(f)} \exp (i \theta)]$, where uniformly distributed random phases $\theta$ were added to the power spectral density $S(f)$ (which is the Fourier transform $S(f)=\mathcal{F}(C(\Delta E)$ ) according to the Wiener-Khinchin-Theorem). Adding random phases $\theta$ imitates the basically random changes of the accumulated phases along the multiply scattered paths through the system. The enlargement shows that the conductance curve becomes smooth on small scales.

\subsubsection{Fractal Fluctuations: Semiclassics}

We have seen that the conductance through a mesoscopic cavity as a function of an external parameter (e.g. a gate voltage or a magnetic field) shows reproducible fluctuations caused by quantum interference. In the following, we will give a short overview into the semiclassical theory [17], that predicts fractal conductance fluctuations in chaotic systems where the underlying phase space is mixed. Note that in an experiment, chaotic systems typically exhibit a mixed phase space e.g. due to the influence of the magnetic field or due to a soft-wall potential (in contrast to ideally specularly reflecting hard walls).

Where does the fractality emerge from? If the average of the phase gain $\theta$ accumulated on the different paths traversing the system exists, the conductance curves are smooth on parameter scales that correspond to a change of the average phase gain on the order of and smaller than the wave length of the carriers as we have seen in Fig. 4.4. In systems with mixed phase space, where chaotic and regular motion coexist, the phase gain of trajectories traversing the system, however, is typically algebraically distributed

$$
P(\theta) \propto \theta^{-\gamma}, \text { where } \gamma<2
$$

and no average phase gain exists (neglecting the finiteness of the coherence length and assuming the semiclassical limit $\hbar_{e f f} \rightarrow 0$; The transition to the quantum mechanical regime, i.e. the role of the finite $\hbar_{e f f}$, is discussed in [18]). Therefore, the conductance curve of such a system fluctuates on all parameter scales and forms a fractal. Ketzmerick has shown, that under these conditions the conductance curve possesses the statistical properties of fractional Brownian motion [17]. The fractal 


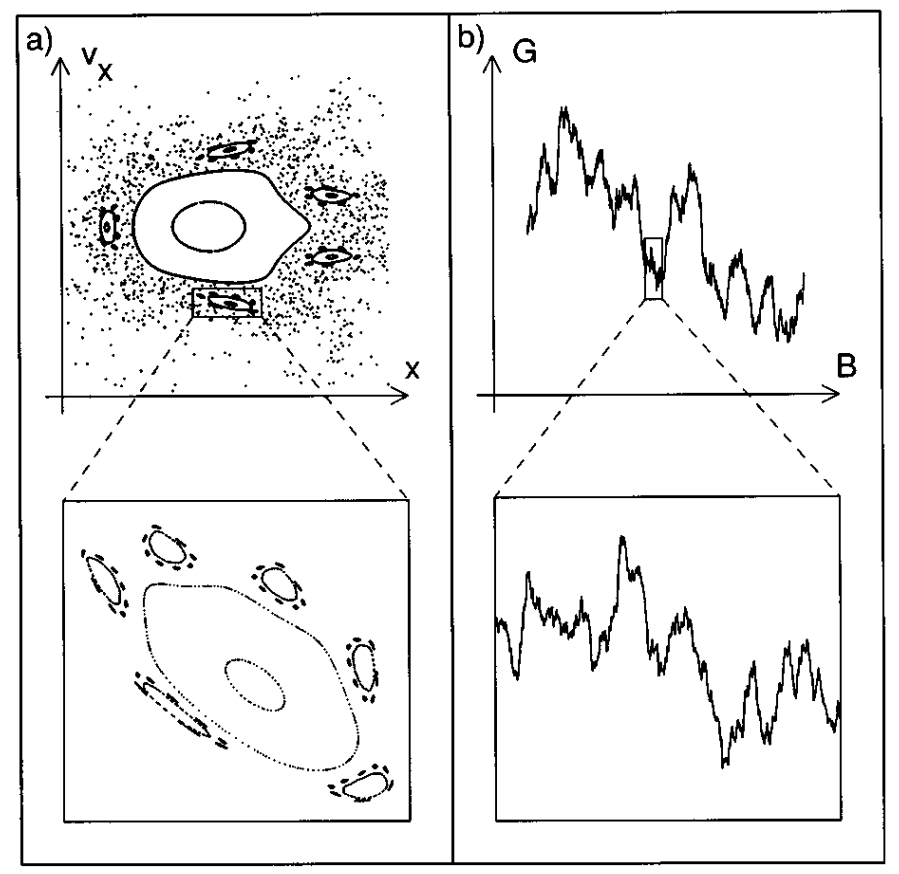

Figure 4.5: (a) A typical hierarchical mixed phase space structure of a 2D chaotic system and (b) FCF are shown. The figure is taken from ref [17]. The Poincaré surface in (a) shows for the example of an antidot array the intersections of one chaotic and 6 regular trajectories with the $\left(y=0, v_{y}>0\right)$ plane [113. The conductance fluctuations shown in (b) are described by fractional Brownian motion $(D=1.4)$.

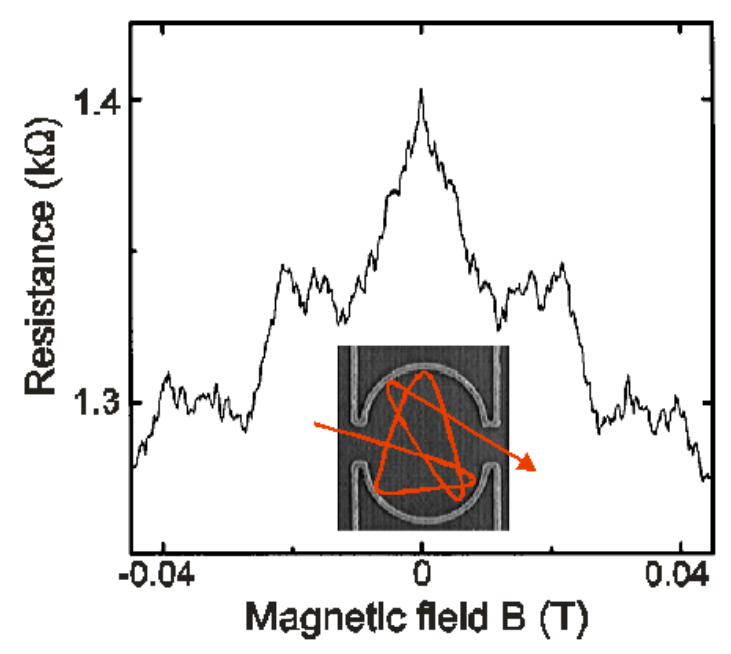

Figure 4.6: Resistance (i.e. inverse conductance) versus magnetic field for the open stadium billiard at a gate voltage of $21.9 \mathrm{~V}$ and at $50 \mathrm{mK}$. The 2DEG was $95 \mathrm{~nm}$ below the surface. Fluctuations of the resistance on both large and small scales can be seen. The inset shows a scanning electron micrographs of a device similar to the one that was used. Schematically, a single classical trajectory through the stadium is drawn. The figure is adapted from A. Sachrajda et. al. [19]. 
dimension $D$ is connected to the exponent $\gamma$ of the algebraic distribution of phase gains by

$$
D=2-\frac{\gamma}{2}
$$

Due to the algebraic distribution of the phase gain $P(\theta)$, the autocorrelation function exhibits a cusp

$$
C(\Delta E)=C(0)-(\Delta E)^{\gamma},
$$

in contrast to the Lorentzian shape of the correlation function for UCF where the corresponding conductance curve becomes smooth on small parameter scales. The semiclassical argument leading to the cusp was confirmed experimentally in a onedimensional periodic array of potential wells [114]. An alternative to reasoning with the average phase gain is to discuss the staying probability [17]. In a mixed phase space, the staying probability $P(t)$ decays algebraically (with the same exponent $\gamma$ as for the distribution of the average phase gain) as chaotic trajectories in the vicinity of the islands can get caught for long times in a scale-free manner in the hierarchy of cantori [36, 37, 115] - see Sec. 2.2 for an introduction. (We already encountered the self-similar hierarchy of islands in a mixed phase space in Sec. 3.7 , where we investigate the stability of discrete breathers consisting of BECs in optical lattices.)

An example of experimental evidence for the fractal behavior of the conductance is shown in Fig. 4.6. In that work the conductance through a stadium billiard with lithographic radius of $1.1 \mu \mathrm{m}$ was measured as a function of an external magnetic field perpendicular to the plane of the billiard. The cavity was defined using metallic gates on a high mobility AlGaAs/GaAs wafer. The high mobility material provides the required long phase coherence length. The device leads were made especially wide $(0.7 \mu \mathrm{m})$ in order to allow most trajectories to rapidly exit the stadium, with the exception of those trajectories which were trapped near the hierarchical phase space structure at the boundary between regular and chaotic motion. This feature and the high mobility made this a suitable device for the observation of fractal conductance fluctuations and explains, why in earlier experiments on conductance fluctuations in ballistic microcavities [15] no fractals were seen.

\subsubsection{Experiments Beyond Semiclassics}

Due to the quantum nature of the FCF it came as a surprise when recent experiments indicated that decoherence does not necessarily destroy the fractal nature of the conductance curve but may only change its fractal dimension [116, 117]. Namely it was found that with decreasing coherence length the scaling region over which the fractal was observed did not shrink - as would be expected from the semiclassical arguments -, but that the fractal dimension changed. The results of the experiment can be seen in Fig. 4.7. A decrease in temperature enlarges the electron phase coherence length and is therefore expected to induce more quantum mechanical conduction. In addition, a decrease in cavity size should increase the influence of coherent transport processes.

In other words, from bottom to top in Fig. 4.7, the transport is expected to be more and more classical. However, still the conductance curve retains its fractality, 


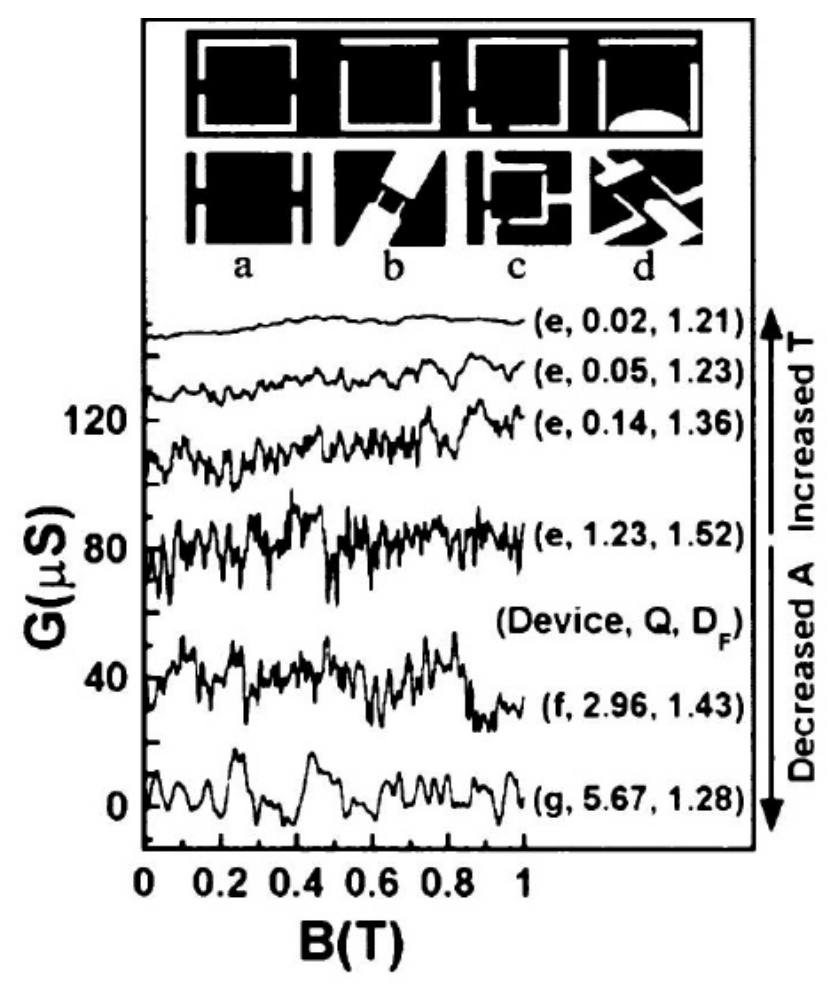

Figure 4.7: Schematics (not to scale) of the gate patterns (top row) together with the equivalent scanning electron micrographs (second row from top) for several billiards. Bottom: Magnetoconductance fluctuations measured for billiards e, $\mathrm{f}$, and $\mathrm{g}$ (see trace labels). Billiards e, f, and $\mathrm{g}$ have mean free path $4.4 \mu \mathrm{m}$ and the same geometry as b but are different sizes. All billiard openings are $0.2 \mu \mathrm{m}$ wide. The traces are offset vertically for clarity. From top to bottom the temperature is decreasing for the first four curves $(4.2 \mathrm{~K}, 1.4 \mathrm{~K}, 0.48 \mathrm{~K}, 0.03 \mathrm{~K})$ at constant cavity size $1.0 \mu \mathrm{m}^{2}$, while for the lower three curves at constant temperature $(0.03 \mathrm{~K})$ the cavity size decreases from top to bottom $\left(1.0 \mu \mathrm{m}^{2}, 0.36 \mu \mathrm{m}^{2}, 0.16 \mu \mathrm{m}^{2}\right)$. The last number in the right-hand brackets indicates the box-counting dimension $D$. Figure taken from [116].

though the fractal dimension decreases. This finding is quantified in 4.8(right), which shows that with decreasing quantum lifetime of states the fractal dimension of the curves decreases as well, however, a change of the fractal scaling range (e.g. between the point DB2 and DB3 in Fig. 4.8(left)) was not observed. These experiments seem to partly contradict the semiclassical theory of fractal scaling and inspired our investigations shown in the next chapter.

\subsubsection{Fractal Fluctuations: Classical Origin}

\subsubsection{Numerical Evidence}

As a starting point of our investigations and to connect it to the experiments we numerically study the classical conductance through a rectangle (hard-wall) and a stadium billiard (soft-wall) as a function of a perpendicular magnetic field as shown in Fig. 4.9. (Throughout this chapter, we will study the transmission, which, in accordance with the Landauer theory of conductance, is proportional 

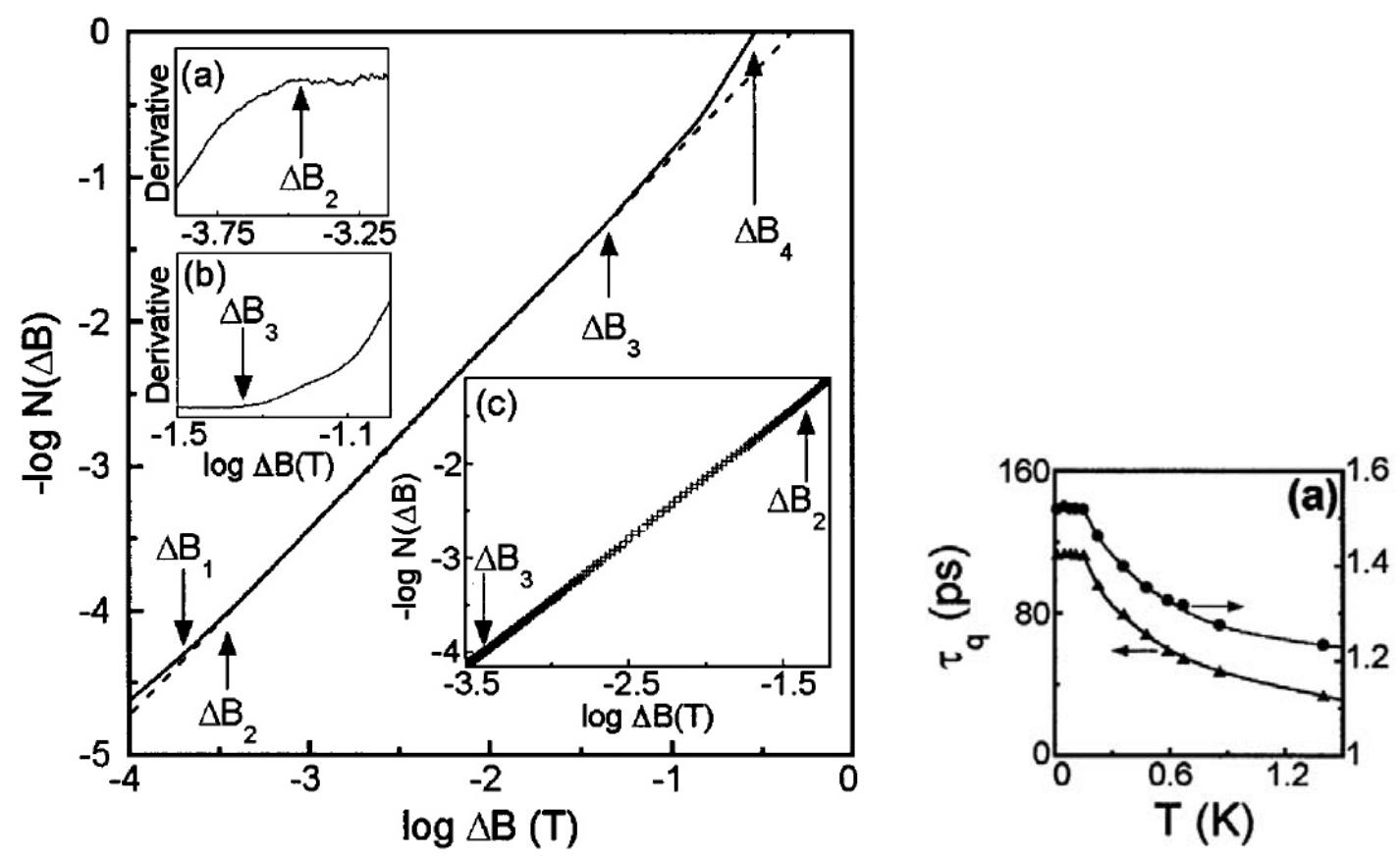

Figure 4.8: (left) Box-counting analysis of magnetoconductance curves. The data (solid line) follow a straight line (dashed line) between the magnetic field scales DB2 and DB3 while the gradient of the line gives the fractal dimension of the curve under investigation. The insets (a) and (b) are derivative plots of the data and (c) shows the individual data points (for clarity only every tenth point is plotted). (right) Lifetime of quantum states $\tau_{q}$ (lower curve) and fractal dimension (upper curve) as a function of the temperature. The figures are taken from ref. [116].

to the conductance, see e.g. [108].) Note that not only the phase space of the stadium but also of the rectangle billiard is mixed in the presence of a perpendicular magnetic field. In both cases, fractal analysis clearly reveals the fractal nature of the conductance curves. As the simulation is purely classical, the fractal scaling cannot be caused by interference effects.

\subsubsection{Model: Open Standard Map}

So what is the underlying mechanism for the fractality of the conductance curve and how can we understand its dimension? To study this mechanism in detail we will, because of its numerical and conceptual advantages, analyze the transport in Chirikov's standard map [39, 118, 119] (see Sec. 2.2.1). This paradigmatic system shows all the richness of Hamiltonian chaos. And since - as will become apparent below - our theory relies only on very fundamental properties of chaotic systems, it is a natural choice as a model system. The standard map is defined by

$$
\begin{aligned}
\theta^{\prime} & =\theta+p^{\prime} \\
p^{\prime} & =p+K \sin \theta
\end{aligned}
$$

with momentum $p$, angle $\theta$ and the 'nonlinearity parameter' $K$, which drives the dynamics from fully integrable $(K=0)$ to fully chaotic $(K \gtrsim 8)$. In between the 

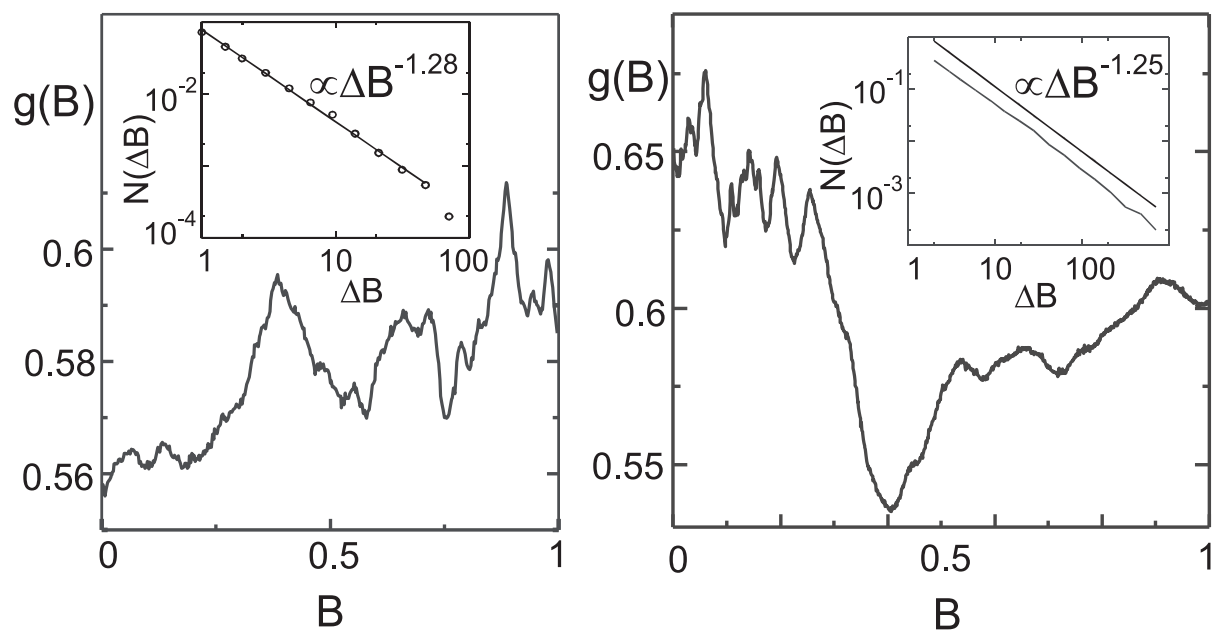

Figure 4.9: Classical Conductance $g(B)$ through a stadium (left, geometry as in ref. [19]) and square billiard (right, geometry as in ref. [116]) versus magnetic field $B$. Both fluctuating conductance curves are fractals, as is shown in the insets by the result of the box-counting analysis (see text). Their respective dimensions are $D \approx 1.28$ for the stadium and $D \approx 1.25$ for the square billiard.

phase space is mixed. The standard map can be interpreted as the Poincaré surface of an autonomous conservative system of two degrees of freedom. As such the map can by viewed to directly correspond to the Poincaré map at the boundary of a chaotic ballistic cavity, connecting it conceptually with the experimental system. We introduce absorbing boundary conditions (see e.g. ref. [120]), i.e. when $p$ exceeds (drops below) a maximum (minimum) threshold value, the particle is transmitted (reflected) and leaves the cavity. As can be seen right from the definition of the standard map, the envelope of the entryset (which is the phase space projection of the injection lead) is simply half a period of a sine function times $K$.

A trajectory entering the system eventually contributes either to the total transmission or reflection, and we mark the corresponding point in the entryset by a color code (transmission: red, reflection: blue) as shown in Fig. 4.10. Chaotic dynamics, through its fundamental property of stretching and folding in phase space, leads to a lobe structure (see Fig. 4.11 (bottom)), which is typical for chaotic systems and not special to the standard map. The lobe structure is translated into transmission by summing up the intersections of the transmission lobes along a horizontal line, see Fig. 4.11. A lobe of thickness $w$ gives rise to a maximum contribution $\Delta T \propto w^{\beta}$ with $\beta=0.5$. Variation of the external parameter $K=7.55$ leads to a fractal transmission curve $T(K)$ with $D \approx 1.25$. Note that with $K=7.55$ we reside in a regime near to the first fundamental accelerator mode which terminates at $K=7.45$. However, trajectories in tiny accelerator mode islands which still might exist, exit the phase space region quickly, and do not affect the long-time survival statistics, thus making the system effectively fully chaotic.

An analysis of the distribution of the widths $w$ of lobes exhibits a power law

$$
n(w) \propto w^{-\alpha}
$$




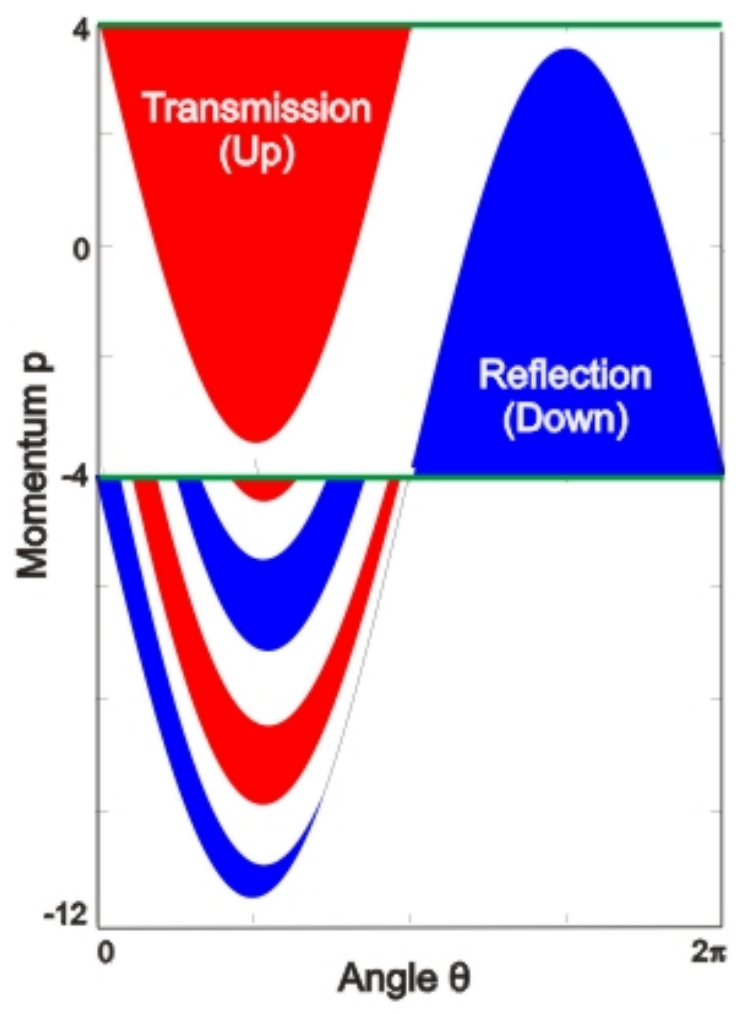

Figure 4.10: Illustration of the absorbing boundaries (green horizontals), the transmission and reflection areas of the standard map and the phase space projection of the injection lead (entryset). The entryset is shown after two iterations of the standard map.

In the next chapter, we will analytically relate the power law exponent of lobe widths to the fractal dimension of the conductance curves.

\subsubsection{Analytics: Fractal Dimension}

How does the fractal dimension depend on the power law distribution of lobe-widths and the curvature of the lobes? To this aim, we study a random sequence of curve segments mimicking the intersection of consecutive lobes of widths $w$, distributed algebraically with exponent $\alpha$ and curved like $w^{\beta}$. We define $X_{i}:=\sum_{j=1}^{i} w_{j}$ and

$$
T(X)=(-1)^{i}\left(X-X_{i}\right)^{\beta} \quad: \quad X_{i}<X \leq X_{i+1} .
$$

An example of this curve of "random lobes" with $\alpha=1.9$ and $\beta=\frac{1}{2}$ is shown in Fig. 4.12 (top). The box-counting analysis clearly reveals a fractal structure.

We further simplify the problem by replacing the lobes by a sequence of stripes of widths $x$ with power law distribution $n(x) \propto x^{\alpha}$. Dispensing with the sign of the fluctuation, the transmission reads

$$
\left.T(X)=\left(X_{i+1}-X_{i}\right)^{\beta}\right)^{\beta} \quad: \quad X_{i}<X \leq X_{i+1} .
$$

This yields histogrammatic transmission curves $T(X)$ like the bottom curve of Fig. 4.12. As shown in the inset, the fractal dimension of the resulting transmission curve remains unchanged compared to the corresponding calculation with random 

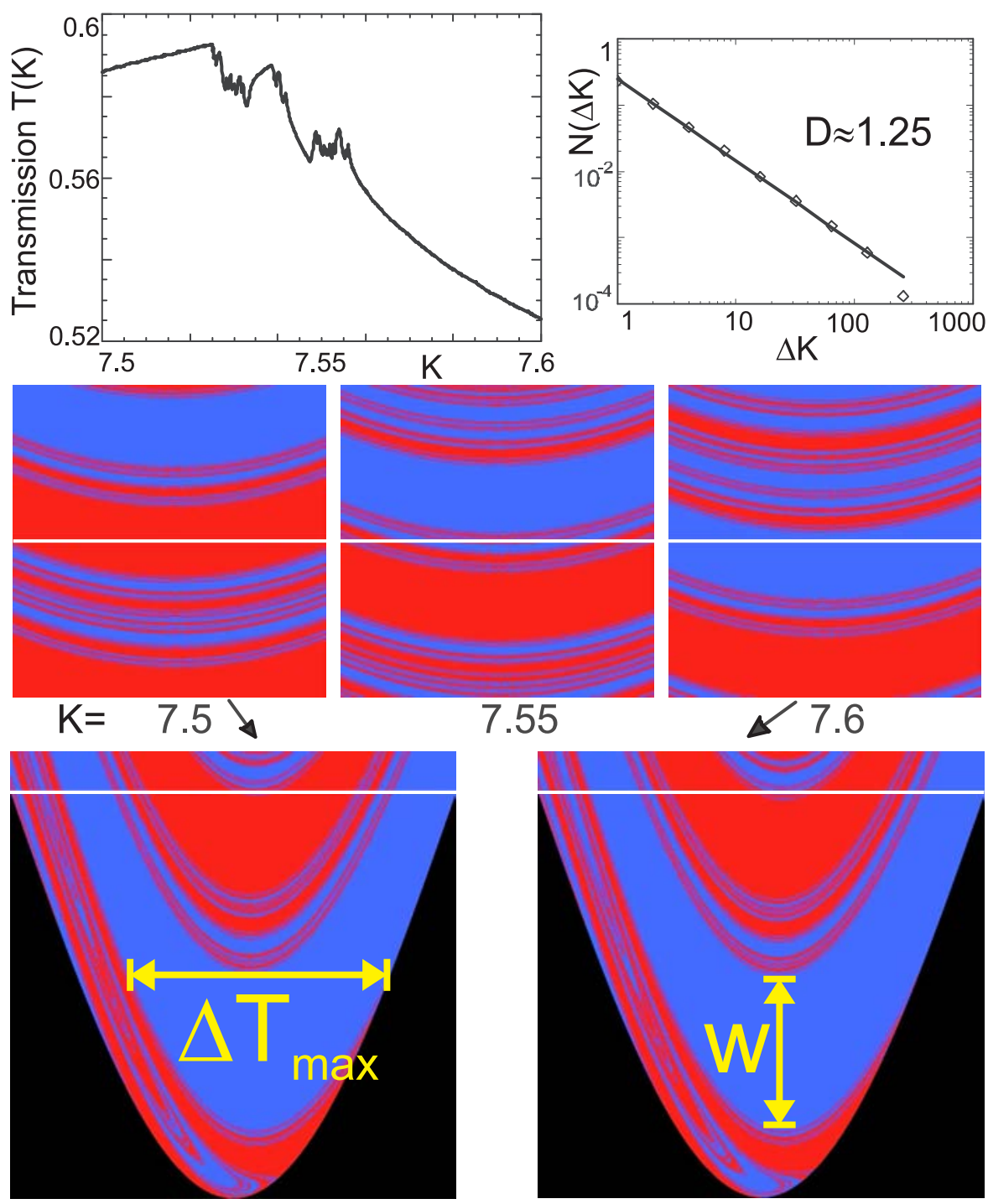

Figure 4.11: How lobes translate into fluctuations: In the lower row the entryset of the standard map with absorbing boundary conditions at $\pm 3 \pi$ for $K=7.5$ and $K=7.6$ resp. can be seen. The three pictures in the center row show the magnification of the central sections of the entryset for three different values of $K=7.5,7.55$ and 7.6. The transmission $T(K)$ for $K=7.5 \ldots 7.6$ is shown in the top left picture. Note that a small change in $K$ shifts the lobes vertically, but conserves the overall phase space structure, and that the largest fluctuations are caused by intersection with the apex of lobes. Starting from $K=7.5$, a large transmission lobe is cut by the horizontal line (see text), i.e. the transmission increases with $K$. In the same way, e.g. the fluctuations of $T(K)$ near $K=7.55$ can be understood. The box-counting analysis reveals a fractal structure (top right). 


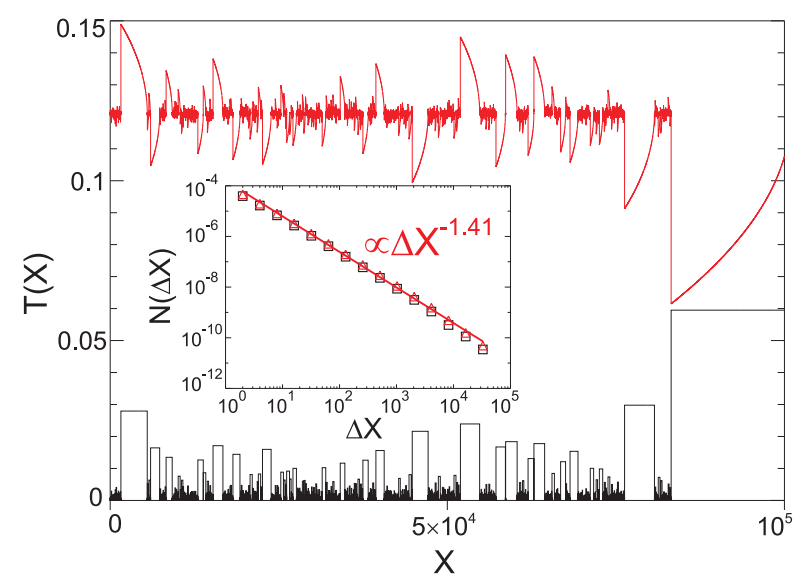

Figure 4.12: Transmission $T(X)$ for lobes (red upper curve, shifted along the y-axis for clarity) and stripes (black lower curve) for one and the same random distribution with $\alpha=1.9, \beta=0.5$. The inset shows the box-counting analysis for the upper (red triangles) and lower transmission curve (black squares). The regression line is drawn for the upper curve, whose fractal dimension is 1.41 .

lobes within the precision of the box-counting analysis. Thus, the measured fractal dimension of the curve does not change when considering stripes instead of lobes and also when neglecting the sign of each contribution, confirming the intuition, that the fractal dimension depends only on the relative scaling, i.e. $\alpha$ and $\beta$, but not on the detailed form of the curve sections.

For these curves like the bottom one of Fig. 4.12 with $\alpha-\beta>1$, we can give an analytical expression for the fractal dimension and then estimate the fractal dimension of the transmission curve in the standard map. We apply the boxcounting method, which we therefore review shortly (see Sec. 2.3 and ref. [42] for a more detailed introduction). In this approach the fractal curve lying in a $n$-dimensional space is covered by a $n$-dimensional grid. Let the grid consist of boxes of length scale $s$. The box-counting dimension is then given by

$$
D=-\lim _{s \rightarrow 0} \frac{\log N(s)}{\log (s)}
$$

where $N(s)$ is the number of non-empty boxes. For our problem, we divide $N(s)$ into three contributions $N(s)=n_{a}+n_{b}+n_{c}$, as schematically drawn in Fig. 4.13A. The number $n_{a}$ of vertically placed boxes (see mark (a)) covering contributions from stripes of widths $x>s$, reads

$$
n_{a}(s) \propto \frac{1}{s} \int_{s}^{\infty} p(x) x^{\beta} d x \propto s^{-(\alpha-\beta)} .
$$

Secondly, the number $n_{b}$ of horizontally placed boxes covering horizontal contributions of stripes of widths larger than $s$, see Fig. 4.13A(b), is given by

$$
n_{b}(s)=\frac{1}{s} \int_{s}^{\infty} p(x) x d x<\frac{1}{s} \int_{0}^{\infty} p(x) x d x .
$$




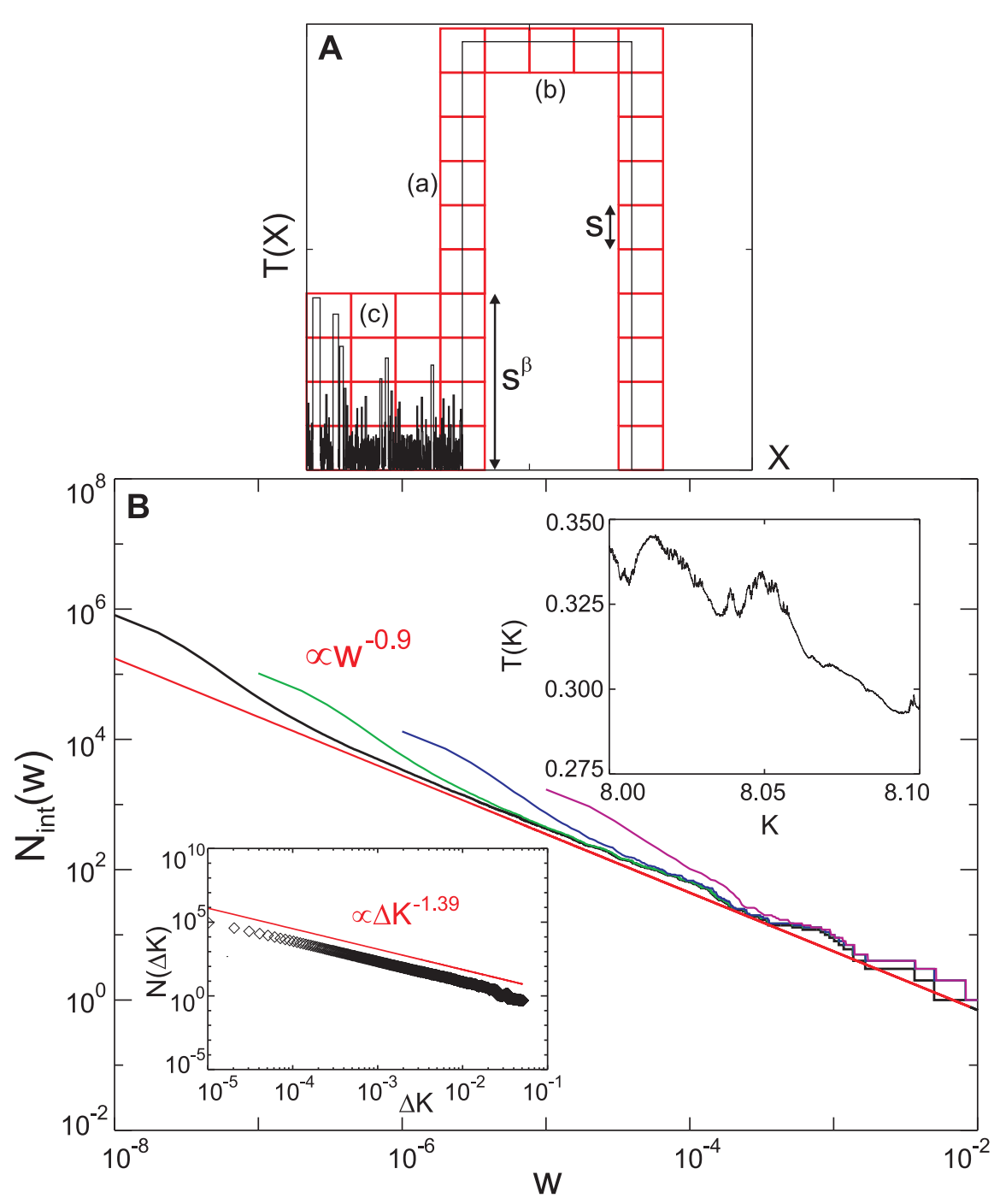

Figure 4.13: A. Schematic transmission according to Fig. 4.12 (bottom), covered with boxes of size $s$. There are three contributions marked (a-c). B. Total number $N_{\text {int }}(w)=$ $\int_{w}^{\infty} n\left(w^{\prime}\right) d w^{\prime}$ of lobes (for the open standard map with $|p|<4 \pi$ ) of width larger than $w$ on a double logarithmic scale. The four curves show estimates for increasing resolution $w_{\min }=10^{-5}$ (pink) $\ldots 10^{-8}$ (black). The curves clearly approach a power law corresponding to $n(w) \propto w^{-1.9}$. The insets show the transmission curve $T(K)$ for values $K=8.0 \ldots 8.1$ calculated from $2 \times 10^{13}$ trajectories and its fractal dimension. 


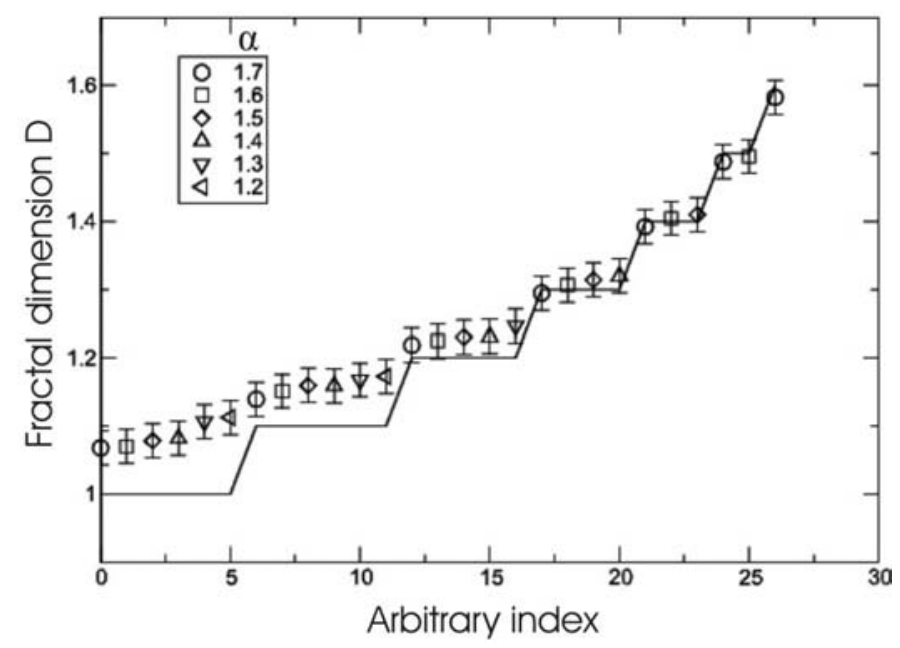

Figure 4.14: Fractal dimension of transmission curves based on random lobes according to Eq. 4.12. In the legend, $\alpha$ is given, while $\beta$ varies from $0.1,0.2, \ldots, 0.6$, e.g. the three data points around $D=1.4$ correspond to $(\alpha, \beta)=(1.7,0.3),(1.6,0.2)$ and $(1.5,0.1)$. For $D \gtrsim 1.2$ the data points lie on plateaus confirming the analytical relation $D=\alpha-\beta$. The same results within the accuracy of the fractal analysis are obtained when calculating the transmission according to Eq. 4.7. The error bars in the figure are an estimate and are of total size 0.025 .

Hence $n_{b}$ scales like $s^{-1}$ and can be neglected in comparison to $n_{a}$ because of $\alpha-\beta>1$. Finally, we determine an upper estimate for the number $n_{c}$ of vertically placed boxes covering the contribution from stripes of widths $x \leq s$. The total length of these widths is $L(s)=\int_{0}^{s} p(x) x d x$, therefor $L(s) / s$ boxes are needed to cover the length. Inflating all heights of the stripes $x \leq s$ to the maximum possible size $s^{\beta}$, see Fig. 4.13A(c), we find

$$
n_{c}(s)<\frac{L(s)}{s} \frac{s^{\beta}}{s} \propto s^{-\alpha+\beta} .
$$

For $s \ll 1$ thus the dominant terms is $n_{a}(s)$. With Eq. 4.8, $N(s)$ gives rise to the box-counting dimension

$$
D=-\lim _{s \rightarrow 0} \frac{\log s^{-\alpha+\beta}}{\log s}=\alpha-\beta
$$

A numerical calculation of the fractal dimension of transmission curves based on random lobes for various pairs of $(\alpha, \beta)$ shows good agreement with the analytical result for $\alpha-\beta \gtrsim 1.2$, as can be seen in Fig. 4.14 .

To connect the analytical result with the calculations of the transmission of the open standard map, we numerically estimate the distribution of lobe-widths in the entryset as shown in Fig. $4.13 \mathrm{~B}$ for $K=8$, where we obtain $\alpha \approx 1.9$. Together with $\beta=1 / 2$, corresponding to first order Taylor expansion of the cosine function, Eq. 4.12 predicts a fractal dimension $D \approx 1.4$. Direct analysis of the transmission curve (see insets of Fig. $4.13 \mathrm{~B}$ ) yields a fractal dimension $D \approx 1.39$, in good agreement with the expected value. 


\subsubsection{Origin of the Power Law}

How can a power law distribution of lobe widths emerge in a fully chaotic open system? Rather one might have expected to find an exponential distribution of lobes in a fully chaotic system. To see why the distribution is algebraic, however, let us examine the simplest case of an open chaotic area preserving map the dynamics of which is governed by a single, positive homogeneous Lyapunov exponent $\lambda$. In each iteration phase space structures are stretched in one direction by $\exp (\lambda)$, shrunk by $\exp (-\lambda)$ in the other and then folded back. The entryset of the open system is thus stretched into lobes of decaying width $w\left(t_{i}\right) \propto \exp \left(-\lambda t_{i}\right)$. The phase space volume flux out of the system decays exponentially as it is typical for a fully chaotic phase space, i.e. $\Gamma\left(t_{i}\right) \propto \exp \left(-t_{i} / \tau\right)$, with (mean) dwelltime $\tau$. The area $\Gamma\left(t_{i}\right) \Delta t$ is the fraction of the exitset that leaves the system at time $t_{i}$. With $t_{i}(w) \propto-\ln (w) / \lambda$ the number of lobes of width $w$ in the exitset is

$$
\mathcal{N}(w) \propto \frac{\Gamma\left(t_{i}(w)\right) \Delta t}{w} \propto \frac{1}{w} \exp \left(\frac{\ln (w)}{\lambda \tau}\right)=w^{\frac{1}{\lambda \tau}-1} .
$$

This suggests that the power law distribution of lobe widths is a generic property even for fully chaotic systems. A quantitative expression for the exponent, however, is not as easy to derive, as e.g. the Lyapunov exponent for the standard map is not homogeneous. Note that we showed the argument for the exitset and not for the entryset for the sake of clarity. A corresponding relation for the algebraic distribution of lobe widths in the entryset can be derived easily by studying the time-reversed map, which again is a chaotic map with the same properties.

\subsubsection{Conclusions}

We have shown that the conductance of purely classical (i.e. incoherent) lowdimensional Hamiltonian systems very fundamentally exhibits fractal fluctuations, as long as transport is at least partially conducted by chaotic dynamics. Thus mixed phase space systems and fully chaotic systems alike generally show FCF. More specifically, we have shown that transport through chaotic systems due to the typical lobe structure of the phase space in general produces fractal conductance curves, where the fractal dimension reflects the algebraic distribution of widths of lobes $P(w) \sim w^{-\alpha}$ in the exit- / entryset. We showed analytically that under these conditions the fractal dimension reads $D=\alpha-1 / 2$.

To connect with the semiclassical picture of FCF, note that an implicit assumption of the semiclassical theory is that the classical dynamics remains unchanged as the external parameter is varied and thus only phase changes are relevant. In many experimental setups, however, the external parameter is changed considerably, leading to very noticeable changes in the classical dynamics. Moreover, we have shown that the classical chaotic dynamics itself already leads to fractal conductance curves! From this follows that even on very small parameter scales the fluctuations due to changes in the classical dynamics are important. In contrast to the semiclassical effect the size of the fluctuations is not universal but depends on specific system parameters. Due to the fractal nature of the classical conductance, however, there is no parameter scale that separates coherent and incoherent fluctuations. 
In conclusion we are led to the following scenario: In general the conductance curve of low-dimensional Hamiltonian systems is a superposition of two fractals one originating in interference which is suppressed by decoherence to reveal the fractal fluctuations reflecting the changes in the classical phase space structure. 


\subsection{Long-range Correlations in Music Rhythms}

A completely different system where fractal fluctuations are found, is musical rhythm played by humans. While in the case of fractal conductance fluctuations, we are interested in the structures on smaller and smaller scales, here the longtime correlations lead to the fractal nature of the fluctuations. Still, the idea of investigating human rhythms emerged from studying FCF.

Music rhythms performed by humans will always exhibit a certain amount of fluctuation compared with an exact rhythm (e.g. given by the steady beat of a metronome). We refer to these fluctuations as temporal deviations which denote the occurrence of individual sounds slightly before or after the exact point in time (see Eq.4.13). It is believed that the task of synchronizing the movement of one's finger to a periodic environmental signal (finger tapping) shows deviations from the signal which exhibit long-range correlations ( $1 / f^{\beta}$-type) [121 124. But musicians do not restrict themselves to finger tapping. Are long-range correlations (LRC) in complex music rhythms played by humans generic?

Long-range correlations $\left(1 / f^{\beta}\right.$-type) are ubiquitously found in various scientific disciplines, examples include DNA sequences [125], condensed matter [126, 127], econophysics [128], human coordination [129, 130], astronomy [131, 132, neurophysics (see e.g. [133, 134] and references therein), etc. General concepts [36, 100, 135. aim at explaining the omnipresence of $1 / f^{\beta}$ noise in nature.

Specifically, one encounters $1 / f^{\beta}$ noise in heartbeat intervals [45, 136-138] and in loudness and in pitch fluctuations in music and speech [26, 27, 139]. The pitch fluctuations were measured by the rate of zero crossings of the audio signal, which, in terms of music, approximately follows the melody. $1 / f^{\beta}$ noise in both loudness and pitch fluctuations was used for stochastic composition which was judged by most listeners to be more pleasing than that obtained using white noise or $1 / f^{2}$ noise [26, 27].

In this section, we show that the temporal fluctuations in simple as well as in more complex music rhythms are generic in the sense that Gaussian $1 / f^{\beta}$ noise is produced, no matter if the task is accomplished with a finger, a hand, a stick, a foot, a combination of these or the voice [31]. The exponent $\beta$, however, depends on the individual and on the specific task. We found a variety of exponents in the range $0.2<\beta<1$.3. In other words, we are dealing with fractional Gaussian noise in the time domain of human music rhythms, which is of stochastic nature and, in contrast to conductance fluctuations in mesoscopic systems, not reproducible. The fluctuations that we focus on comprise of generic (natural) deviations from a given rhythmic pattern - in contrast to intentional accentuations. Accentuation facilitates the interpretation of a music piece and thus plays an important role; however, this is not treated here.

The fact that long-memory processes arise in a broad variety of complex rhythmic tasks suggests that a common basic neurophysical mechanism accounts for generic creation of human rhythms. It is most likely that $1 / f^{\beta}$ noise in music rhythms is not generated in the course of the movement of a limb but due to a neuronal network that is responsible for perception and estimation of time. See [140 142] for an overview on neurophysical modeling of rhythmic timing and estimation of time intervals in the millisecond regime. We will briefly review an idea 


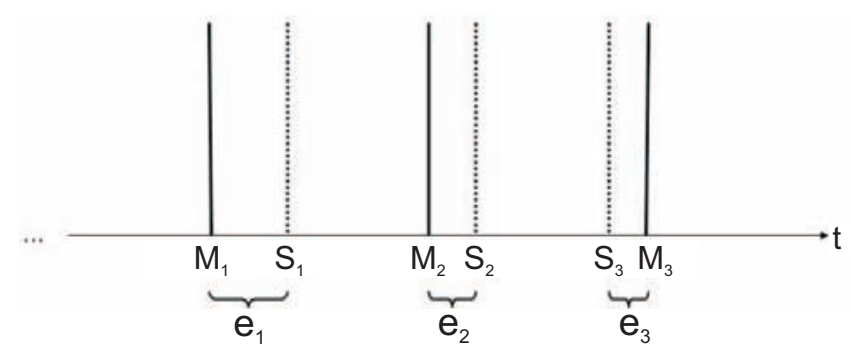

Figure 4.15: Schematic plot of a rhythmic music sequence (e.g. a drum sequence) and a metronome, only pertaining to the temporal occurrence of sounds. The drum sequence with beats at times $S_{n}$ is compared to the metronome $M_{n}$ yielding errors $e_{n}=S_{n}-M_{n}$.

based on a simple stochastic model [143] which generates pulse trains exhibiting $1 / f^{\beta}$ noise in Sec. 4.2.3.

Moreover, in Sec. 4.2.4 we are led to an application of our findings by addressing the following question: Does the rhythmic structure of a piece of music sound better when it is as exact as possible or are 'natural' deviations (comprising longrange correlations) in music rhythms more favorable? Professional audio software applications include a so called 'humanizing' feature, which adds deviations $e_{n}$ (also referred to as errors, see Eq. 4.13 to a given audio sequence. Hence, there exists a desire to generate or modify music on a machine that sounds more natural. We analyzed the humanize tool of such contemporary software applications and found that e.g. for the program 'Pro Tools 1 it consists of Gaussian white noise. So how does a music piece humanized with Gaussian $1 / f^{\beta}$ noise sound? We show with simple computer-generated drum rhythms, that the $1 / f^{\beta}$ humanized music (with $\beta \approx 1$ ) was rated significantly better by professional musicians from the Göttingen Symphonic Orchestra than the conventional humanized sequences comprising a Gaussian white noise error source with the same standard deviation. However, the simple drum rhythms used in the experiments sound rather artificial and the exact rhythm was preferred over the humanized versions.

For more realistic experiments on the influence of LRC in music rhythms on the perception of humans, a music piece (more specifically a pop song) was created, recorded and humanized in cooperation with Cubeaudio recording studio (Göttingen). Experiments with test subjects were carried out in the course of an interdisciplinary diploma thesis in psychology [32] (see Sec. 4.2.4.3). Applications are possible in humanizing music sequences with Gaussian $1 / f^{\beta}$ noise, e.g. in the field of electronic music or in post-production of real recordings, where a patent in the EU and the USA was applied for (Sec. 4.2.4.4).

\subsubsection{Error Time Series}

In this section, we will make the basic definitions needed and give an illustrative example of the error time series originating from a recording of human rhythmic drumming.

\footnotetext{
${ }^{1}$ Pro Tools is widely used by professionals throughout the audio industries worldwide for recording and editing in music production, film scoring, post production etc.
} 


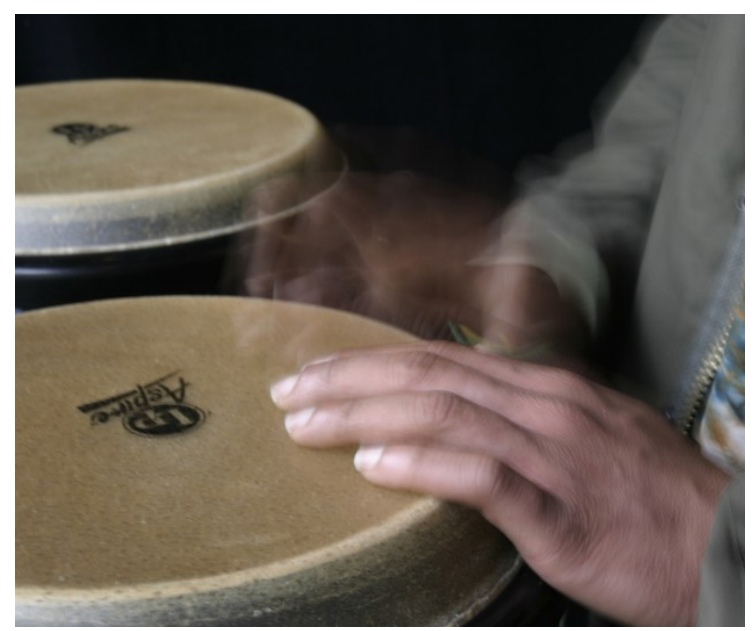

Figure 4.16: Recording beats on a drum. A subject, here a professional drummer, is playing on a drum. The sounds are recorded with a microphone at sampling rate 44.1 khz. Photo taken from www.hippocritz.com by courtesy of Agbenyega Attiogbe.

A simple way to define the deviations of human drumming from a rhythmic reference pattern is to take a metronome as a reference (see Fig. 4.15). Given a 'metronome' $M_{n}=M_{0}+n T$, where $T$ denotes the time interval between metronome clicks and $n=0,1,2 \ldots$ is an integer. And given a (recorded) rhythmic music sequence with sounds (also referred to as beats) at times $S_{n}$, then the error time series is defined as

$$
e_{n}=S_{n}-M_{n},
$$

where $e_{n} \ll T \forall n$. A sound is given by the audio signal $A(t)$ (e.g. the voltage input to a loudspeaker) and as such is extended in time. We define the occurrence of a sound at time $S_{n}$ by

$$
A\left(S_{n}\right)=\max (A(t))
$$

which is well-defined in particular in drum recordings (Fig. 4.16) due to the compact shape of a drum sound $A(t)$ : The envelope of $A(t)$ rises to a maximum value ("attack phase") and then decays quickly ("decay phase") [144]. Thus, if the drum sounds are well separated, a unique maximum $A\left(S_{n}\right)$ can be found. In contemporary audio editing software, typically the onset of a beat is detected [144, 145], which is very useful when beats overlap or when dealing with distorted sequences. In Sec. 4.2.4.2 we will use onset detection to find the temporal occurrences of sounds for humanizing music sequences.

The definition Eq. 4.13 can be easily generalized to considering deviations of a sequence from a complex rhythmic pattern instead of from a metronome. The time between two successive sounds $\tau_{i}=S_{i+1}-S_{i}$ (also called interbeat intervals) is connected to the timing errors by

$$
\tau_{i}=T+e_{i+1}-e_{i}
$$

In the following let us define the terms $1 / f^{\beta}$ noise and long-range correlations. Given a discrete stochastic process $x(t)$, customarily, one speaks of $1 / f^{\beta}$ noise, 
when

$$
S(f)=|\mathcal{F}(x(t))|^{2} \propto f^{-\beta} \text { and } 0<\beta<2,
$$

where $\mathcal{F}$ denotes the Fourier transform. Note that there are as well definitions where $0<\beta<1$, see e.g. [146]. In literature, both notations $1 / f$ and $1 / f^{\beta}$ noise are found for the same entity. For simplicity, from now on we will drop the exponent and speak of $1 / f$ noise meaning $1 / f^{\beta}$ noise with $0<\beta<2$. For $\beta=0$ the time series $x(t)$ is uncorrelated (white noise), while for $\beta=2$ it corresponds to Brownian motion (integrated white noise). In addition, when the $x(t)$ are Gaussian distributed, it is called Gaussian $1 / f$ noise (also referred to as fractional Gaussian noise).

A (discrete) long-memory process is defined as a stochastic process where the autocorrelation function $C(k)=<x(t) x(t+k)>$ corresponding to a time series $x(t)$ decays to zero so slowly that it is not summable (in the discrete case) [121, 146]:

$$
\sum_{k=0}^{\infty} C(k)=\infty,
$$

which holds, if

$$
C(k) \propto k^{-\gamma} \text { and } 0<\gamma<1,
$$

also referred to as long-range correlations (LRC). Finally, for $0<\gamma<1$, Eqs. 4.16 and 4.17 are connected by the Wiener Khinchin theorem

$$
S(f)=\mathcal{F}(C(k))
$$

where $S(f) \propto f^{\gamma-1}$ for a long-memory processes.

A simple example of a recording and its processing is shown in Figs. 4.16 and 4.17. Here, the given task was to follow the clicks of a metronome given over headphones with a hand on a drum at 180 beats per minute (bpm). The output is recorded with a microphone. A comparison of $S_{n}$ with the metronome (Eq. 4.13) leads to the error time series $e_{n}$. As we see from Fig. 4.17b, $e_{n}$ fluctuates around a mean of $-16.4 \mathrm{~ms}$. In other words, the negative value means that the subject anticipates the next metronome click.

In Fig. 4.18 the power spectral density $S(f)=\left|\mathcal{F}\left(e_{n}\right)\right|^{2}$ of our example is shown. The frequency range under consideration is $f_{\text {min }}<f<f_{\text {nyquist }} / 2$, where $f_{\text {nyquist }}=0.5 \mathrm{sec}^{-1}$ is the Nyquist frequency (i.e. half the sampling frequency of the error time series). The corresponding time window reads $T_{\min }<T<T_{\max }$, where $T_{\min }=\left(f_{\text {nyquist }} / 2\right)^{-1} 60 / 180=1.3 \mathrm{sec}$ and $T_{\max }=333 \mathrm{sec}$. A least squares fit of the PSD in the considered frequency range indicates that $S(f)$ can be approximated by a power law

$$
S(f) \propto f^{-\beta}
$$

with exponent $\beta \approx 1.2$. However, to closely investigate the long-range behavior of the time series, we used several methods described in Sec. 4.2.2.

\subsubsection{Data Set \& Analysis}

The data set analyzed in Fig. 4.21 consists of the recorded output of several rhythmic tasks performed by humans, which can be divided into three subsets $\mathcal{S}_{\text {drum }}, \mathcal{S}_{\text {sing }}$ 

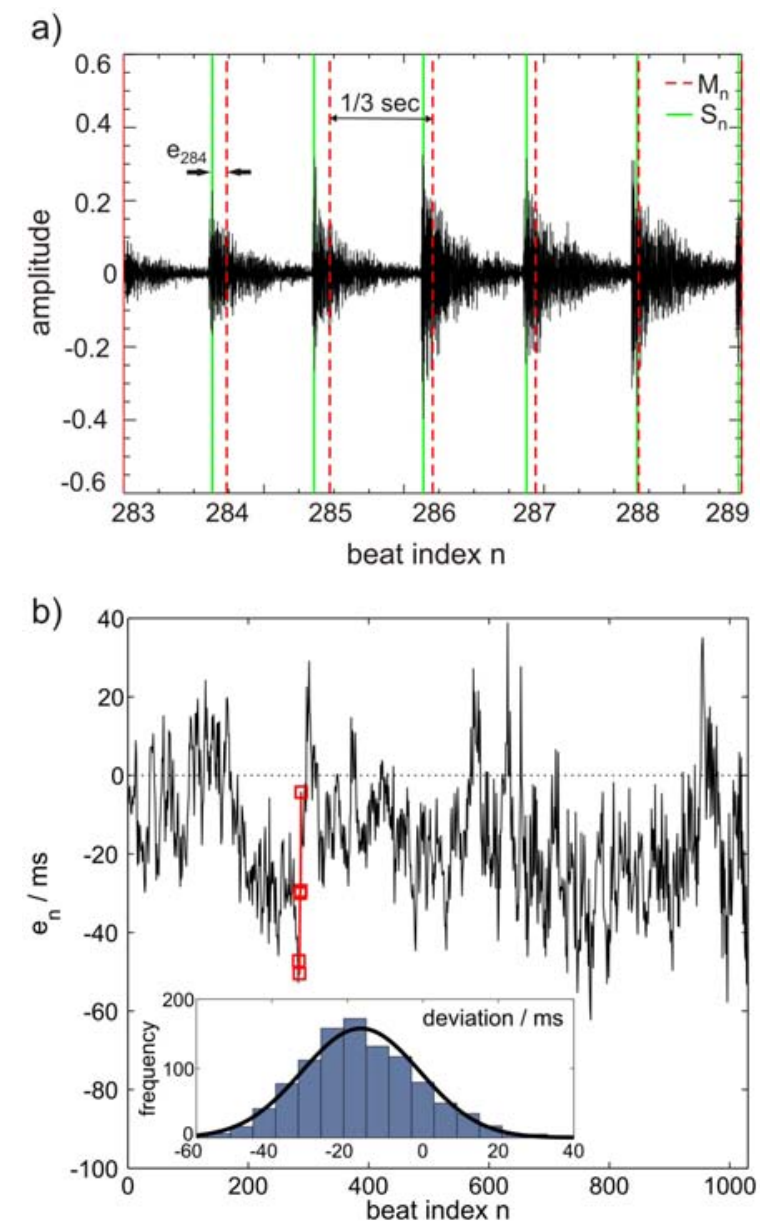

Figure 4.17: (a) The drummer in Fig. 4.16 was recorded in tapping with the hand on the drumhead of a drum according to the clicks of a metronome, that were given over headphones. Shown is a section of the recorded amplitudes (e.g. the voltage input signal to a loudspeaker) over the beat index $n$. A beat-finder detects the temporal occurrence of the beats (green lines), here given by the maximum of the amplitudes. The metronome (red dashed lines) has $180 \mathrm{bpm}$, i.e. two consecutive clicks are separated by $1 / 3 \mathrm{sec}$. (b) Corresponding error time series of the recording. The errors $e_{284}$ to $e_{288}$ depicted in Fig. $4.17 \mathrm{a}$ are marked with red squares. Note that values $e_{n}<0$ indicate that the sounds occur earlier than the metronome click. Inset: The pdf of the time series is well approximated by a Gaussian distribution in agreement with a previous analysis of finger tapping data [121]. The Gaussian distribution in this example has mean $-16.4 \mathrm{~ms}$ and standard deviation $15.6 \mathrm{~ms}$. 


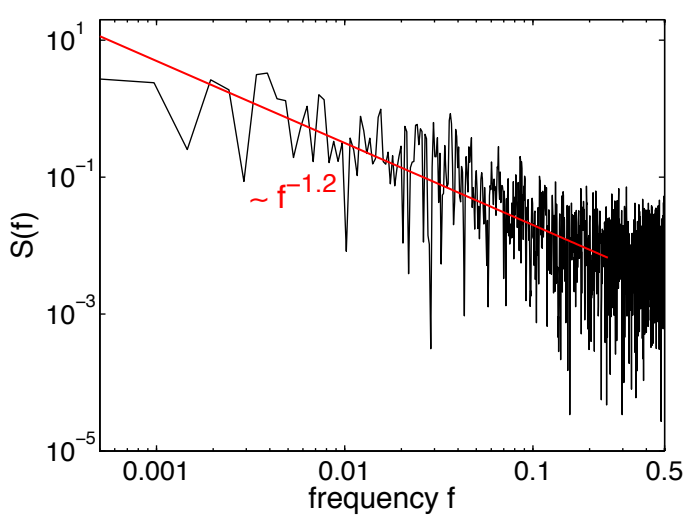

Figure 4.18: Power spectral density (PSD) of the time series in Fig. 4.17. Note that this figure serves only as an illustration - for clear statistical estimates several methods from time series analysis are applied in Sec. 4.2.2. The PSD indicates $1 / f^{\beta}$ noise at low frequencies in the range $f_{\min }<f<0.25=f_{\text {nyquist }} / 2$ with $\beta \approx 1.2$. Note that the logarithmic transform does not preserve the structure in the variance of the estimates at each frequency. Estimates at frequencies with high amplitudes have lower relative variances than the relative variances at frequencies with low amplitudes. Since in $1 / f$ noise, low frequency is associated with high amplitudes, this produces the triangular structure, where the bottom part of the envelope descends more steeply than the top part. An averaged PSD of the same time series analyzed here is shown in Fig. 4.19b (second curve from top).

and $\mathcal{S}_{\text {tap }}$. All subsets have in common that a human was given metronome clicks over headphones. To address the task that was recorded, leading to an error time series $e_{n}^{\mathcal{I}}$, we annotate each error time series with a task index $\mathcal{I}$, which denotes the specific task explained in the following (e.g. $\mathcal{I}=(1,2)$ corresponds to tasks 1 and 2 in Fig. 4.21).

The subset $\mathcal{S}_{\text {drum }}$ consists of drum recordings provided by a recording studid 2 and of recordings that we made. During professional drum recordings in a studio, a drummer is sitting in front of a drum set and uses both arms and the feet to generate a complex music rhythm that, typically, is part of a piece of music. The feet are used for operating pedals connected to bass drum and hi-hat (Fig. 4.23). During a studio recording it is customary, that the drummer hears metronome clicks over headphones while playing. We make use of this fact to analyze the error time series of professional drumming data. Given the speed of the song, usually measured in beats per minute (BPM), a comparison of the drum recordings with the metronome can be drawn. In detail, the subset $\mathcal{S}_{\text {drum }}$ comprises error time series $e_{n}^{\mathcal{I}}$ obtained from several recordings of different tasks $\mathcal{I}=(1-7)$ :

- $e_{n}^{(1,2)}$ : Studio data of 2 drummers belonging to different music bands playing different patterns at $190 \mathrm{bpm}$ and $132 \mathrm{bpm}$. The drum sequences analyzed each are part of a music song.

- $e_{n}^{(3-5)}$ : Short rhythmic pattern at $124 \mathrm{bpm}$. The pattern (given in $4 / 4$ bars)

\footnotetext{
${ }^{2}$ Cubeaudio Recording Studio, Göttingen, Germany
} 
contains quarter and eighth notes, is of 2 bars length and is repeated continuously by the drummer.

- $e_{n}^{(6,7)}$ : Tapping with a drum stick on a drum according to the clicks of the metronome at $124 \mathrm{bpm}$.

More specifically, $e_{n}^{(3-5)}$ originate from the same recording, but the time series $e_{n}^{(3)}$ is obtained by comparison with a $248 \mathrm{bpm}$ metre (thus focusing on the deviations in the eighth notes), while $e_{n}^{(4)}$ is obtained by comparing the beats with respect to the metronome at $124 \mathrm{bpm}$. In $e_{n}^{(5)}$ the off-beats are analyzed (i.e. by comparison with a metronome at $124 \mathrm{bpm}$ that is shifted by $T / 2$ ). To summarize, the complexity of the rhythmic patterns in the subset $\mathcal{S}_{\text {drum }}$ decreases from $e_{n}^{(1,2)}$ over $e_{n}^{(3-5)}$ to $e_{n}^{(6,7)}$. At the one end there are real drum recordings that are part of popular music songs, while at the other end a link to finger tapping analyzed in literature [121 124] is made.

Secondly, the subset $\mathcal{S}_{\text {sing }}$ consists of 4 recordings $(\mathcal{I}=(8-11))$ of short rhythmic sounds of the voice according to a metronome at $124 \mathrm{bpm}$. We chose short phonemes (such as ['dee']) to obtain well-peaked amplitudes of the beats. However, the fact that long-range correlations are found is expected to be independent of the phoneme that is articulated.

Finally, the subset $\mathcal{S}_{\text {tap }}$ comprises of tapping experiments $(\mathcal{I}=(12-27))$ similar to what can be found in literature, but to connect with the subset $\mathcal{S}_{\text {drum }}$ we recorded tapping with a finger or a hand on the drumhead of a drum. We recorded tapping at two different metres for each test subject: $124 \mathrm{bpm}$ (even task index) and 180 bpm (odd task index) ${ }^{3}$. We discarded the first 50 beats to eliminate transients. When a deviation is larger than $100 \mathrm{~ms}$, than that beat is called a 'glitch' and is omitted, which happened in less than 5 out of 1000 cases for the recordings in $\mathcal{S}_{\text {sing }}$ and $\mathcal{S}_{\text {tap }}$ and does not appear to affect the long term behavior we seek to quantify. Test subjects in $\mathcal{S}_{\text {tap }}$ and $\mathcal{S}_{\text {sing }}$ were of mean age $33 \pm 9$ yrs.

We used several methods to analyze the correlation properties of the data, namely the periodogram (also referred to as PSD), detrended fluctuations analysis (DFA), and the maximum likelihood estimation (MLE [147], details are given below). See Sec. 2.3.1 for a short introduction to fractal analysis.

For the estimate via the periodogram, a least square fit of the power spectral density $S(f)=\left|\mathcal{F}\left(e_{n}\right)\right|^{2}$ is made on a double logarithmic scale $4^{4}$ As the variance of the PSD is large (at high frequencies), an average of the PSD is taken (Fig. 4.19). For the averaging, the time series is divided into boxes of length $L$, and in each box the arithmetic average is taken. We used $L=5$ and checked that moderate averaging $L \leq 10$ does not notably alter the resulting exponent.

The second method, DFA, is applied directly in the time domain, see Sec. 2.3.1 and [45, 149] for details. The integrated time series is divided into boxes of equal length $s$. DFA involves a detrending of the data in the boxes using a polynomial of degree $k$. Then the sum $F(s)$ of the fluctuations over the trend is calculated.

\footnotetext{
${ }^{3}$ Only exception are tasks 14 and 15 which both are recordings at $124 \mathrm{bpm}$.

${ }^{4} \mathrm{~A}$ comparison of the linear regression with a band integrated regression, where the points in the power spectrum are equidistant, shows that the linear regression leads to more reliable results [148].
} 

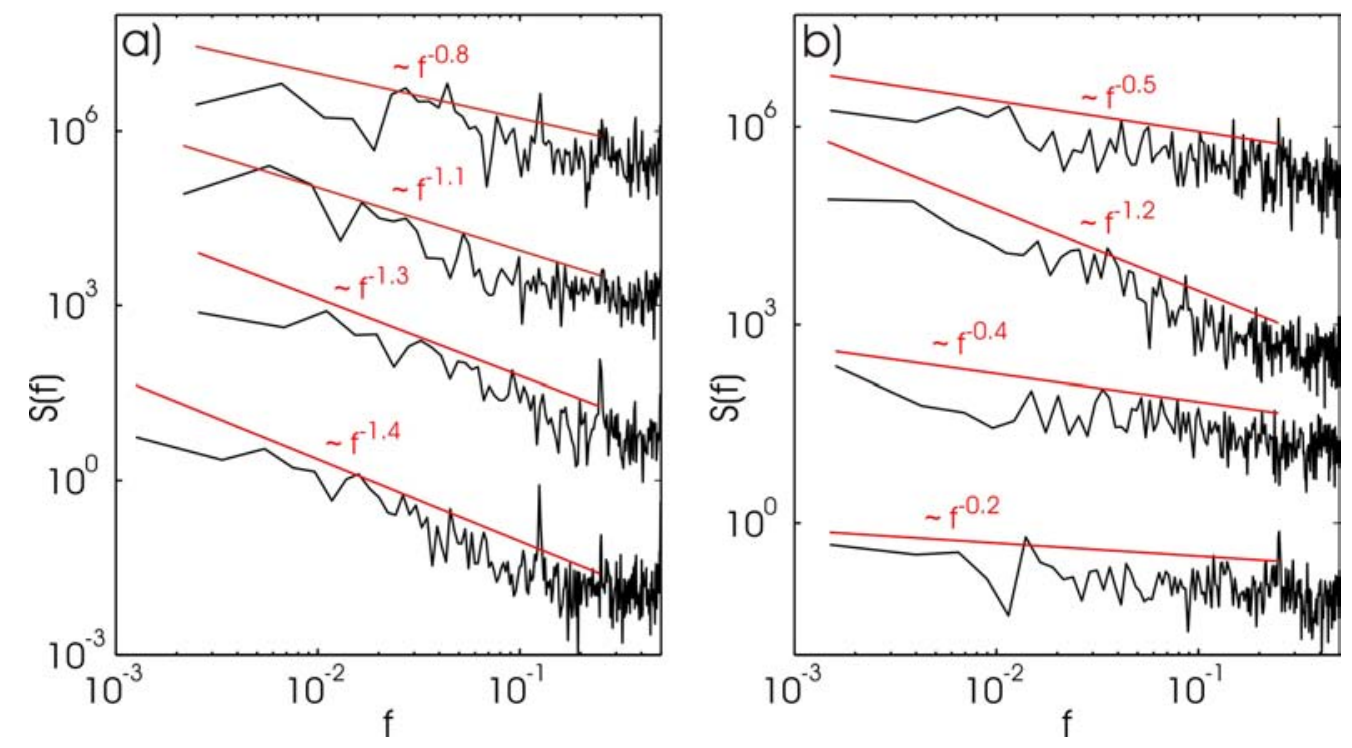

Figure 4.19: Examples of the averaged PSD of different error time series of the data set. The red straight lines are least square fits in the regime $f_{\min }<f<f_{\text {nyquist }} / 2$, thus indicating a long-range behavior of the correlations. (a) Analysis of different complex drum sequences. The upper two curves originate from the analysis of drum sequences of two different songs provided by a recording studio. For the lower two curves we made recordings of a drummer continuously repeating a drum pattern. (b) Examples from the PSD of $\mathcal{S}_{\text {sing }}$ and $\mathcal{S}_{\text {tap }}$. The upper two curves show the PSD for tapping on a drum for two different professional drummers. The lower two curves show the analysis of recordings of short rhythmic sounds of the voice according to a metronome. Subjects were a professional drummer and a professional a-capella singer.

Again, a linear relationship on a log-log plot indicates the presence of power law (fractal) scaling $F(s) \sim s^{\alpha}$. The scaling exponent $\alpha$ is related to the PSD exponent $\beta$ by [146]

$$
\beta=2 \alpha-1
$$

In case of fractional Gaussian noise $\alpha=H$ is the Hurst exponent. We calculated the DFA exponents using DFA2, which means quadratic detrending $(k=2)$, see Fig. 4.20, Similar results were obtained with linear detrending (DFA1).

Once it is statistically established by means of PSD and DFA that the spectral density $S(f)$ is well-approximated by a power law, we use the MLE to estimate the exponent $\beta$ and determine confidence intervals. The MLE is applied to $S(f)$ in the same frequency range as for the PSD method (i.e. $f_{\text {min }}<f<f_{\text {nyquist }} / 2=0.25$ ).

\section{Results}

As can be seen in the examples in Figs. 4.19 and 4.20 (task index for top to bottom curves are $\mathcal{I}=(2,1,3,5)$ and $\mathcal{I}=(22,21,8,10)$ in Fig. 4.19a and Fig. 4.19b) the time series exhibit long-range correlations. We find Gaussian $1 / f$ noise for the whole data set; see Fig. 4.21 where the exponents $\beta$ with corresponding confidence intervals are collected. We emphasize that the exponent $\beta$ of the Gaussian $1 / f$ noise is not universal and varies in our data set between $0.2<\beta<1.3$. Note that the ex- 


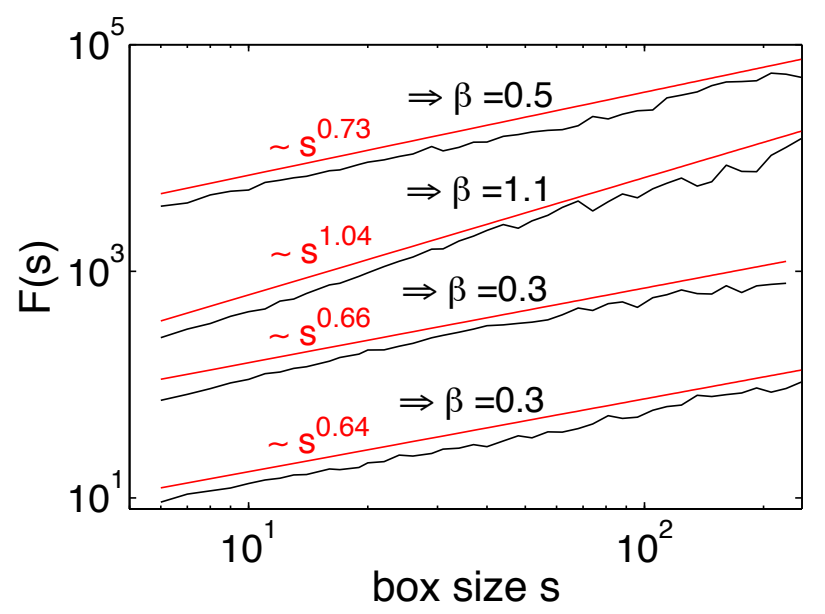

Figure 4.20: Examples of detrended fluctuation analysis (DFA2) for the same error time series analyzed in $4.19 \mathrm{~b}$, i.e. the lower two curves correspond to recordings of short rhythmic sounds of the voice, while the upper two curves originate from tapping on a drum. Clearly, a fractal scaling $F(s) \sim s^{\alpha}$ is found for box sizes in the range $6<s<N / 4$, where $N$ is the total length of the time series. To compare with the exponents $\beta$ calculated in Fig. 4.19 b, the PSD exponents $\beta=2 \alpha-1$ are given as well.

a)

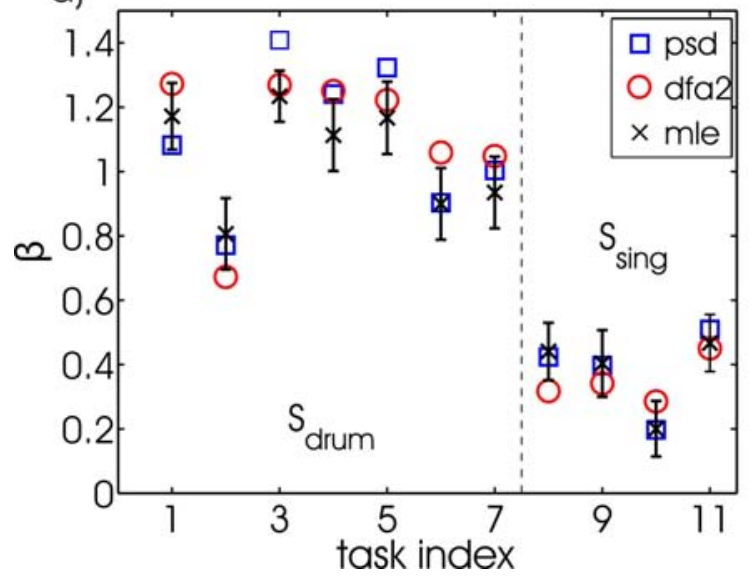

b)

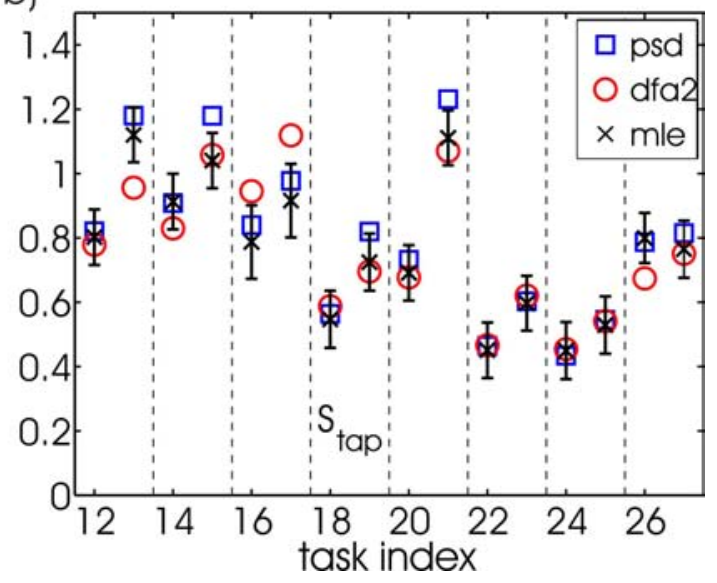

Figure 4.21: Results of the time series analysis of the whole data set obtained from different rhythmic tasks. The tasks correspond to (complex) drum sequences $(\mathcal{I}=(1-7))$, rhythmic vocal sounds $(\mathcal{I}=(8-11))$ and to tapping with a finger or hand on the drumhead of a drum $(\mathcal{I}=(12-27))$. All sequences recorded lead to error time series that exhibit Gaussian $1 / f$ noise. The exponents $\beta$ obtained by the different methods PSD, DFA2 and MLE are compared and show overall good agreement. The confidence intervals are provided by the MLE. The two tasks in-between two neighboring vertical dashed lines are recordings of the same test subject at different metronome tempi (124 bpm and $180 \mathrm{bpm}$ respectively), e.g. tasks 20 and 21 are recordings of the same individual. 
ponent varies as well within recordings of different tasks made by the same person. For example for the tasks $\mathcal{I}=(8,20,21)$ the same person was recorded (who is a professional drummer), where the different MLE exponents $\beta(\mathcal{I})=(0.4,0.7,1.1)$ are obtained.

The tasks in the whole data set are quite diverse and the data set is not large enough to significantly detect task-dependent differences. However, our focus will be on generic long-range correlations in music rhythms that appear in all tasks and with every subject that we recorded (as long as the subject was able to follow the rhythm).

Is the exponent related to the music expertise of the person recorded? In the subsets $\mathcal{S}_{\text {sing }}$ and $\mathcal{S}_{\text {tap }}$ we recorded musicians and non-musicians with different musical experience ranging from laypersons to professionals, where the music expertise was estimated by a self-evaluation. In the subset $\mathcal{S}_{\text {drum }}$ the subjects recorded are drummers, i.e. experienced in playing the drums. A clear dependence between $\beta$ and the music expertise was not found in the data set. A prerequisite for LRC is that the person is able to follow the rhythm. Long-range correlations vanish, if the individual loses the rhythm too often and reenters with help of the metronome we speculate that this resets the memory of the internal mechanism (some kind of "dynamical clock", see Sec. 4.2.3 responsible for the rhythmic timing.

Another considerable quantity is the standard deviation of the error time series (the average for the whole data set is $\bar{\sigma}=21 \mathrm{~ms}$ ). The lowest standard deviations were found for musicians, however a musician does not necessarily intend to minimize the standard deviation. We surmise that the more a person tries to synchronize with the external metronome, which can reduce the standard deviation in case of trained drummers to $\sigma<10 \mathrm{~ms}$, the lower the exponent $\beta$. This suggests that some sense of rhythmic timing is crucial for LRC, where the degree of the correlation shrinks with increasing external influence. In the limit, where the test subject is triggered completely externally (as in reaction time experiments), no LRC are found [122].

We are led to the following conjecture: When a musician is strongly triggered externally (by a metronome or another music sequence) and synchronizes highly with the external signal, the degree of long-range correlations in the corresponding error time series is smaller than in the case when the musician is 'free floating', i.e. triggered mostly internally.

\subsubsection{Modeling Rhythmic Drumming}

It seems most likely that $1 / f$ noise in music rhythms is not generated in the course of the movement of a limb but due to a neuronal network that is responsible for perception and estimation of time. An overview on neurophysical modeling of rhythmic timing and estimation of time intervals in the millisecond regime is found in [140 142, 150]. As described in [140], modeling an internal clock that could account for timing in the millisecond regime has been a challenge for decades, see e.g. [124, 151] for recent approaches. The millisecond regime is defined in [140] as being above $10 \mathrm{~ms}$ and below 500 to $1000 \mathrm{~ms}$. Since experienced drummers achieve standard deviations of below $10 \mathrm{~ms}$, we alter the time scale of interest for our purposes to $5<\mathcal{T}<500 \mathrm{~ms}$. The human reaction time lies within that regime: 


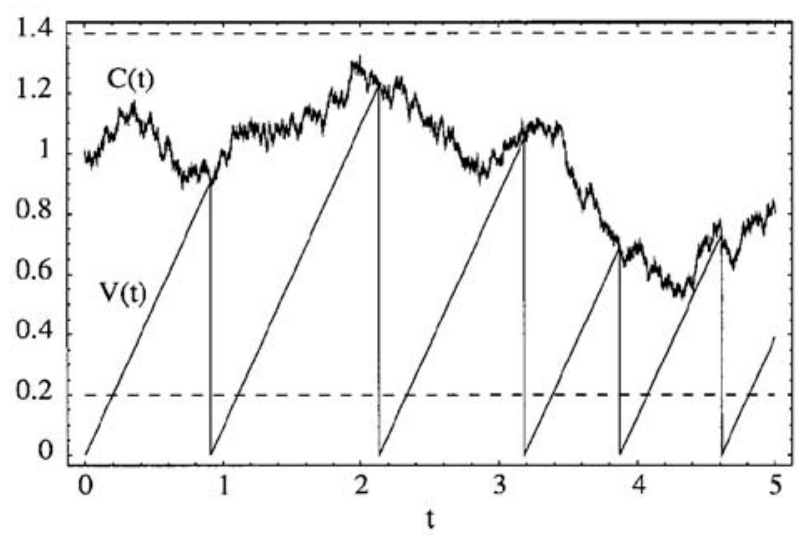

Figure 4.22: Dynamics of a single neuron model which exhibits long range correlated $(1 / f$ type) pulse trains [143. The model is based on an integrate-and-fire mechanism consisting of two variables: A linearly increasing potential $V(t)$ and a threshold $C(t)$ which evolves according to a random walk with diffusion constant $D$ and reflecting boundaries. When the potential $V(t)$ exceeds the threshold, a spike is generated and $V(t)$ is reset. However, the interspike intervals can be approximated by a 1 dimensional map with multiplicative noise (Eq. 4.22), which does not show long range correlations in the $\tau_{k}$.

For the tasks where subjects depress the space bar on a computer keyboard as rapidly as possible after the presentation of an optical stimulus the reaction time has mean $\sim 100 \mathrm{~ms}[122$.

A simple stochastic model for which it can be shown analytically to generate spike trains exhibiting $1 / f$ noise is presented by Davidsen and Schuster [143]. The essential ingredient of the integrate-and-fire model is a fluctuating threshold which performs a Brownian motion. Whenever an increasing potential $V(t)$ hits the threshold, $V(t)$ is reset to the origin and a pulse is emitted (Fig. 4.22). If $V(t)$ increases linearly in time, the interspike intervals (ISI) $\tau_{k}$ can be approximated by a random walk with multiplicative noise [143]:

$$
\tau_{k+1}=\tau_{k}+\sqrt{D \tau_{k}} \xi_{k}
$$

where $\xi_{k}$ denotes the white noise source and $D$ is the diffusion constant. However, there are drawbacks when relating Eq. 4.22 to the interbeat intervals Eq. 4.15. The PSD of the above-mentioned spike trains (which can be idealized to a sequence of zeros and ones, where a 1 indicates the occurrence of a spike) reveals a longmemory process. In contrast, it can be shown that a time series $\tau_{k}$ (Eq. 4.22) does not exhibit long-range correlations. A second problem is, that for the random walk with multiplicative noise, the ISI distribution function $P(\tau)$ is proportional to $\tau^{-1}$ [143], while the pdf of the interbeat intervals in human rhythmic drumming is well approximated by a Gaussian distribution.

In order to develop some further aspects of Davidsen's and Schuster's model, several approaches are possible. One approach is to adaptively change the gradient $a$ of the linearly rising potential of the neuron, trying to keep a mean ISI $\bar{\tau}$. This introduces a second parameter $a$ where the ISI $\tau_{k}$ depend on $a$. Another idea is related to synchronization, or simply to coincidence. For our purposes, consider $N$ neurons described by the single neuron model (Fig. 4.22) which are connected to 
one neuron $\mathcal{A}$, which integrates the incoming spikes of the $N$ neurons and which sends an action potential, if the following condition is fulfilled: $M$ out of $N$ presynaptic neurons fire in the same time window. Note that it was shown in purely random networks, that synchronous spiking activity of subgroups can persist and propagate if the non-additive nature of dendritic input integration has been recently uncovered experimentally is taken into account [152]. We surmise that the condition (i.e. the requirement of coincidence of $M / N$ spikes) for neuron $\mathcal{A}$ to spike alters the distribution of the ISI such that it is different from a power law and may approximate a Gaussian. However, the modeling is still work in progress.

\subsubsection{Humanizing Music Sequences}

As shown in Sec. 4.2.2, we found $1 / f$ noise in the error time series of music rhythms played by humans. In this section, we will consider an application arising from the question, which effect long-range correlations have on the perception of humans.

Computer-generated music, such as an artificial drum sequence, has no difficulty in always keeping the exact beat, since synthesizers and computers are equipped with precise timing mechanisms. But computer-generated music is often recognizable just for this perfection. The idea to artificially add small fluctuations to music sequences is realized in the so-called 'humanizing' feature, which is implemented in contemporary audio software applications. More precisely, humanizing is the procedure of adding an error time series $e_{n}$ (Eq. 4.13) to a given audio sequence. We analyzed the humanize feature which leads to error time series consisting of Gaussian white noise for the program 'Pro Tools' (Version HD 7.4) and of uniform white noise for 'Nuendo 3'. Pro Tools is widely used by professionals throughout the audio industries worldwide for recording and editing in music production, film scoring, post production etc. In terms of humanizing music, we are led to the question:

- How does a piece of music humanized with Gaussian $1 / f$ noise sound in comparison to a piece of music that is either conventionally humanized or exact?

- Are the differences audible to humans?

To investigate these questions, different pieces of music were created and played to test subjects as described in the next sections. For each piece of music three versions were generated which differ only by their error time series $e_{n}$ (Eq. 4.13):

1. Version E ('exact'): $e_{n}=0 \forall n$.

2. Version C ('correlated'): $C_{e}(\tau)=<e_{n}, e_{n+\tau}>=\tau^{1-\beta}$ with exponent $\beta \approx 1$ (thus exhibiting long-range correlations). Also referred to as the Gaussian $1 / f$ humanized version.

3. Version W ('white'): $e_{n}=\xi_{n}$, where $\xi_{n}$ is Gaussian white noise. Also referred to as the Gaussian white noise humanized version. 


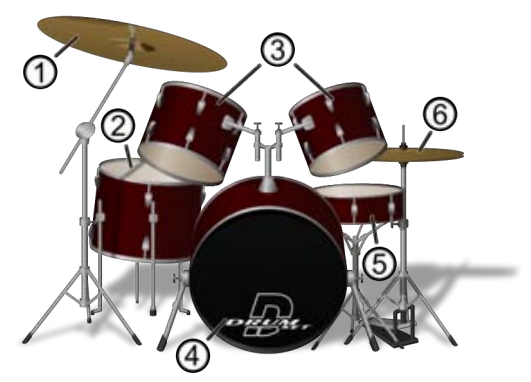

Figure 4.23: Scheme of a drum set with bass drum (4), snare drum (5) and hi-hat (6). Samples of bass drum, snare and hi-hat were used to generate humanized drum rhythms.

All other properties of the versions (such as the pitch and loudness fluctuations which do affect the auditory impression [26, 27, 139]) are identical. To compare the versions $\mathrm{E}, \mathrm{C}$ and $\mathrm{W}$, we choose the same standard deviations $\sigma_{C}=\sigma_{W}$ of the error time series and mean $\left.\left.\left\langle e_{E}\right\rangle=<e_{C}\right\rangle=<e_{W}\right\rangle=0$.

The outline of the following sections is as follows. In Sec. 4.2.4.1 the generation of simple humanized drum rhythms is described and we report the rating of the different versions by test subjects. The creation of a much more complex humanized piece of music is explained in Sec. 4.2.4.2. The rating of the piece of music by test persons is subject of an interdisciplinary diploma thesis in psychology (Sec. 4.2.4.3). Finally, in Sec. 4.2.4.4 a patent for humanizing music sequences is described.

\subsubsection{Drum Rhythms}

We generated simple drum rhythms on the computer, where each drum rhythm consists of 3 different audio samples (bass drum, snare drum, hi-hat - see Fig. 4.23) that are set according to a rhythmic pattern. An audio sample of e.g. a bass drum is a recording of a single bass drum sound. The exact sequence is then humanized to generate versions $\mathrm{C}$ and $\mathrm{W}$ by adding a $1 / f$ or white noise error time series (see Fig. 4.24). Though this is a first demonstration of $1 / f$ humanizing of electronic music, the minimalist way of the generating the music rhythms with only 3 samples makes the rhythmic pieces sound quite monotonous and artificial. Furthermore the music sequences do not contain any variation in timbre of the beats, in loudness or pitch.

For statistical tests, the different versions $\mathrm{E}, \mathrm{C}$ and $\mathrm{W}$ of the drum sequences, each in mono quality and of $\approx 30 \mathrm{sec}$ length, were played in random order (there are subtleties, see Appendix C to 16 professional musicians from the Göttingen Symphonic Orchestra (GSO). The test subjects completed a questionnaire, the main questions are shown in Fig. 4.25. For the experiments with the GSO we used a standard deviation $\sigma_{C}=\sigma_{W}=10 \mathrm{~ms}$. Audio examples (no. 1, 2 and 3 ) of the versions $\mathrm{E}, \mathrm{C}$ and $\mathrm{W}$ with $\sigma_{C}=\sigma_{W}=15 \mathrm{~ms}$ are found as supplementary material to the electronic version of this thesis. To make the differences between the versions more audible, the standard deviation in the audio examples is higher than in the experiments. Still, $\sigma=15 \mathrm{~ms}$ is within the lower range of $\sigma$ measured for music laypersons while finger tapping on a drum (with a finger or a hand). We used a $\chi^{2}$-test with significance level $p_{0}=0.05$, see Appendix $\mathrm{C}$ for details. The outcome 


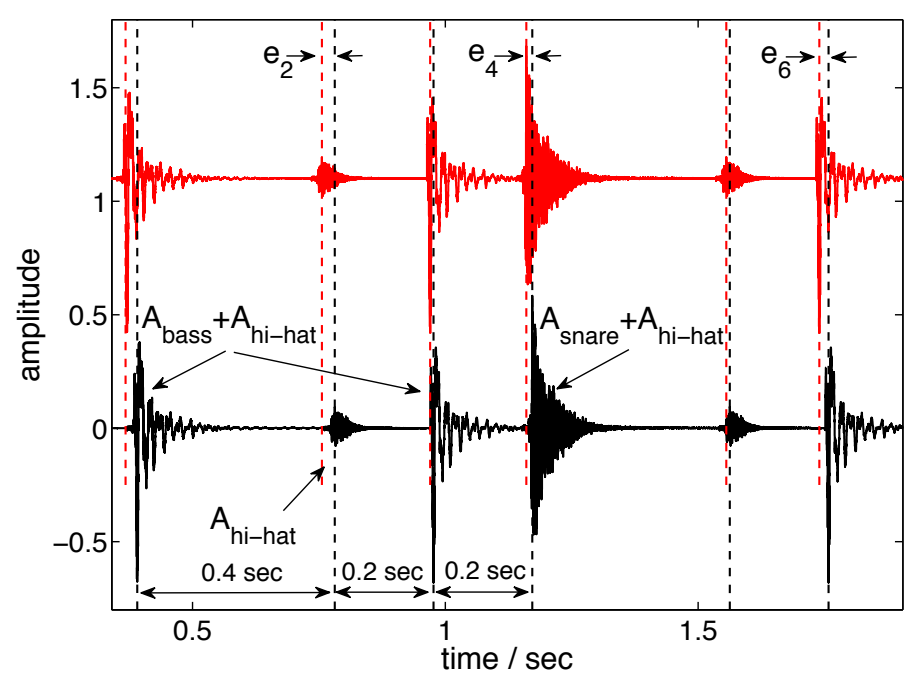

Figure 4.24: How $(1 / f)$ humanizing works. Shown is a section of an exact drum sequence (lower curve) with $153.6 \mathrm{bpm}$ at $4 / 4$ bars and a humanized sequence (upper curve). The length of one quarter note is $0.4 \mathrm{sec}$. The lower and upper sequences correspond to the first $\approx 1.5 \mathrm{sec}$ of audio examples no. 1 and 2 respectively. For $1 / f$ humanizing the upper sequence, to each individual sound at time $S_{n}$ an error $e_{n}$ is added, where $e_{n}$ is computergenerated Gaussian $1 / f$ noise with $\beta \approx 1$. The vertical dashed lines mark the positions $S_{n}$ of the sounds.

1) Are the two samples different?

$$
\text { yes } \mathrm{O} \mathrm{O}_{\text {no }}
$$

2) If you marked "yes" with a cross: Which sample do you like better?

\section{а O O B}

Consider the sample which you like better. How does it sound in comparison to the other sample (please make exactly one cross)?

$$
\begin{aligned}
& \mathrm{O} \text { more precise } \\
& \mathrm{O} \text { more natural }
\end{aligned}
$$

Figure 4.25: The main questions of the questionnaire given to musicians from the Göttingen Symphonic Orchestra. We did not analyze the third question (which version sounds more natural / more precise), as all versions sound rather artificial, due to the minimalist construction of the drum rhythms with 3 samples. 


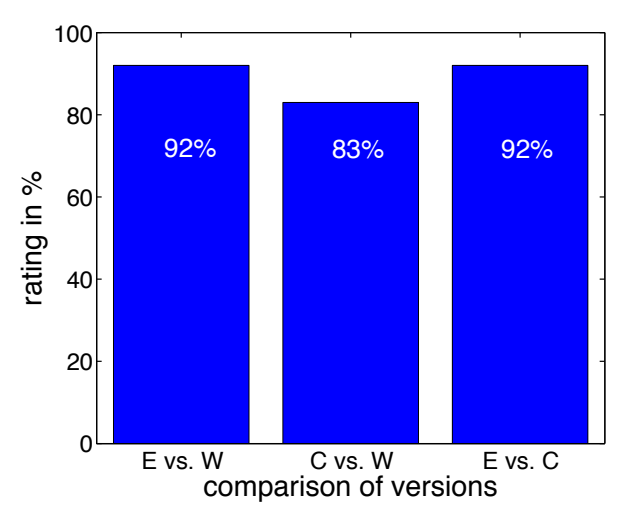

Figure 4.26: "Which version sounds better?" Experiments with 16 musicians from the Göttingen Symphonic orchestra rating the $1 / f$ humanized version $C$ the white noise humanized version $\mathrm{W}$ and the exact version $\mathrm{E}$. Two versions were played after one another (in random order), 10 comparisons were made by each test subject. Version $\mathrm{C}$ was rated significantly better than version $\mathrm{W}\left(p=0.02, \chi^{2}=5.3\right)$ with $83 \%$ vote. A preference was given to the exact version, compared to both the versions $\mathrm{C}\left(p=0.002, \chi^{2}=9.3\right)$ and $\mathrm{W}\left(p=0.002, \chi^{2}=9.3\right)$.

of the experiment with the GSO is the following (Fig. 4.26):

- The $1 / f$ humanized versions are rated significantly better than the (conventional) white noise humanized versions $\left(p=0.02, \chi^{2}=5.3\right)$.

- The exact version was rated best $\left(p=0.002, \chi^{2}=9.3\right)$.

One reason why the exact version is preferred may be due to the artificial character of the rhythmic sequences. Thus, deviations are perceived as failures by the test subjects. The fluctuations in the rhythmic structure might be rated differently when more complex artificial music or music originating from real recordings (that include as well fluctuations in timbre and loudness) is humanized. Though the exact version was rated best, a comment of one of the test subjects from the GSO summarizes our idea of a perceived lack of human touch nicely:

Some samples were so 'overprecise', too regular by intention - that does not sound natural.

\subsubsection{Creating Humanized Music}

In order to compare the version $\mathrm{C}, \mathrm{W}$ and $\mathrm{E}$ in a more realistic way, a pop song called "Everyday, everynight" was created, recorded and humanized in collaboration with Cubeaudio recording studio (Göttingen). The song has $125 \mathrm{bpm}$, approx. 4 mins length and a steady beat in the eighth notes. Hence, the song consists of $\sim$ 1000 beats in the eighth notes. Instrumentation was chosen such that the sequences can be humanized easily, meaning that the sounds occurring at times $S_{n}$ should be rather compact and well separated from each other.

The song, available in stereo quality, consists of a total of $\sim 100$ sequences including several sequences for the drum set (as the output of each part of the drum set, 
e.g. the bass drum, was recorded individually), guitar, bass, vocals and, to a small part, software instruments. Except for the software instruments, the sequences are recordings of professional musicians, where each musician is recorded separately. In the next step, the individual sequences are humanized and finally mixed together. The program 'Pro Tools' was used for onset detection [144, 145] of the beats and for mixing the song. Note that the complexity of the song (consisting of mostly real recordings of several instruments, and including fluctuations of loudness and pitch in $\sim 100$ sequences) is much higher than that of the single drum sequences generated with the computer as described in Sec. 4.2.4.1.

We did not humanize the vocals, which cover the fluctuations of the other instruments to a certain degree. The vocals are identical in all three versions E, $\mathrm{C}$ and $\mathrm{W}$. To generate version $\mathrm{W}$, we did not use the white noise humanize tool implemented in Pro Tools, but we added the white noise error time series manually to have control over the realizations and the standard deviation.

The version $\mathrm{C}$ was humanized with $1 / f^{\beta}$ noise, where $\beta \approx 1$. The standard deviations of the versions $\mathrm{C}$ and $\mathrm{W}$ is $\sigma=15 \mathrm{~ms}$, which is a trade-off between having significant results (for large $\sigma$ ) and a regular (natural) sound (for small $\sigma$ ). The Gaussian was cut at $2 \sigma$, i.e. realizations larger than $2 \sigma$ were omitted in the time series, in order to exclude single rare events that might influence or dominate the judgment of listeners.

Still, there is a main drawback, that give the song an artificial touch. We humanized all $\sim 100$ sequences with one and the same error time series $(1 / f$ or white noise)..$^{5}$ This leads to collective shifts (or delays) in the time domain, which is not natural, as all instruments come in too early or too late at a given time in exactly the same manner.

\subsubsection{Perception of Humanized Music}

How does a piece of music humanized with Gaussian $1 / f$ noise sound in comparison to a piece of music that is either white noise humanized or exact? The aim of the interdisciplinary diploma thesis in psychology that we initiated was to analyze the perception of natural fluctuations in music rhythms using the humanized song "Everyday, everynight". The thesis is written by Anneke Fredebohm at the University of Göttingen (Dept. of Cognitive and Decision Sciences). More specifically, a comparison of the two versions $\mathrm{C}$ and $\mathrm{E}$ is drawn, where audio examples of length $\approx 30 \mathrm{sec}$ were played in random order (there are subtleties, see 32 for the experimental design) to 57 listeners who are mostly part of non-professional choirs in Göttingen. There was no information given in advance to the subjects, in which way the versions differ. The differences between the three versions $\mathrm{E}$ and $\mathrm{C}$ are very small and, moreover, they are covered by the vocals. It can be shown using a $\chi^{2}$-test that 32

- the test subjects could significantly hear the difference between the versions $\mathrm{E}$ and $\mathrm{C}(p=0.015)$,

\footnotetext{
${ }^{5}$ The reason why we used the same time series for humanizing all sequences is because $1 / f$ humanizing is not yet implemented in contemporary audio software applications and thus had to be done manually and double-checked afterwards.
} 
- there was no preference found when comparing versions E and C.

Note that there is a distinct difference to the simple and artificial drum rhythms analyzed in Sec. 4.2.4.1, where a clear preference for the exact version was observed. Hence the rating of the $1 / f$ humanized piece of music (as compared to the exact sequence) was much better than in the case of the less complex drum sequences. The versions, that the test subjects were listening to are found in audio example no. 4: First, the version $\mathrm{C}$ and after a $5 \mathrm{sec}$ break the exact version $\mathrm{E}$ is played.

A comparison of the versions $\mathrm{C}$ and $\mathrm{W}$ is current work in progress (see audio example no. 5, which consists of the version $\mathrm{W}$, a $5 \mathrm{sec}$ break and the version $\mathrm{C}$ ). We expect a preference of version $\mathrm{C}$ over version $\mathrm{W}$, which would mean that in agreement with the previous study described in Sec. 4.2.4.1, rhythms containing LRC are preferred over those containing white noise error time series.

Surprisingly, as stated above, the version containing LRC was not rated better than the exact version. There are several reasons, which could account for that. First, the standard deviation of $\sigma=15 \mathrm{~ms}$ is too large for a popular song (especially if the listeners are experienced musicians). Second, all sequences were (as mentioned above) humanized according the same realization of the error time series, leading to unnatural collective shifts of all instruments (except for the vocals). And third, it is probably a matter of individual taste and may depend on the music genre [139], whether a song or parts of the sequences within should be as exact as possible of whether natural deviations are tolerated and favored. The complete $1 / f$ humanized song with reduced standard deviation $\sigma=7.5 \mathrm{~ms}$, corresponding to quite precisely playing musicians, is attached to the electronic version of this thesis (audio example no. 6).

We made a first step towards the realization of $1 / f$ humanizing of music sequences. To what extent $1 / f$ humanizing will be useful for audio engineers remains to be seen in practice when it is applied in a more sophisticated way to sequences in music pieces.

\subsubsection{Patent}

The Max Planck Society applied for patents in the EU and the USA for humanizing music sequences with error time series consisting of $1 / f$ noise. The title of the application reads: "Method and Device for Humanizing Music Sequences" (EU application no: 07117541.8 (Sept. 2007), US application no: 12/236,708 (Sept. 2008)). A short extract of the application, that was filed in collaboration with Max-PlanckInnovation, is given below.

"The present invention relates to a method and a device for humanizing music sequences. In particular, it relates to humanizing drum sequences. Large parts of existing music are characterized by a sequence of stressed and unstressed beats (often called "strong" and "weak"). Beats divide the time axis of a piece of music or a musical sequence by impulses or pulses. The beat is intimately tied to the meter of the music as it designates that level of the meter that is particularly important, e.g. for the perceived tempo of the music. A well-known instrument for determining the beat of a musical sequence is a metronome. A metronome is any device that produces a regulated audible and/or visual pulse, usually used to 
establish a steady beat, or tempo, measured in beats per minute for the performance of musical compositions. Ideally, the pulses are equidistant. However, humans performing music will never exactly match the beat given by a metronome. Instead, music performed by humans will always exhibit a certain amount of fluctuations compared with the steady beat of a metronome.

Machine-generated music on the other hand, such as an artificial drum sequence, has no difficulty in always keeping the exact beat, as synthesizers and computers are equipped with ultra precise clocking mechanisms. But machine-generated music, an artificial drum sequence in particular, is often recognizable just for this perfection and frequently devalued by audiences due to a perceived lack of human touch. The same holds true for music performed by humans which is recorded and then undergoes some kind of analog or digital editing. Postprocessing is a standard procedure in contemporary music production, e.g. for the purpose of enhancing human performed music having shortcomings due to a lack of performing skills or inadequate instruments, etc. Here also, even music originally performed by humans may acquire an undesired artificial touch.

Therefore, there exists a desire to generate or modify music on a machine that sounds more natural. It is therefore an object of the present invention to provide a method and a device for generating or modifying music sequences having a more human touch. Preliminary results of empirical experiments carried out by the inventors strongly indicate that a rhythm comprising a natural random fluctuation as generated according to the invention sounds much better or more natural to people than the same rhythm comprising a fluctuation due to Gaussian or uniformly distributed white noise with the same standard deviation."

\subsubsection{Conclusions}

When a human plays a music rhythm on an instrument, inherently there are deviations in the time domain from the exact rhythm. We showed that music rhythms performed by humans generically exhibit long-range correlations in the deviations from an exact rhythmic pattern. The deviations are generic in the sense, that the corresponding time series can be described by Gaussian $1 / f^{\beta}$ noise, no matter if the music rhythm is made with a finger, a hand, a stick, a foot, a combination of these or the voice. The exponent $\beta$ is not universal and depends on the task and the subject. Moreover we investigated the influence of long-range correlations in music rhythms on the perception of humans. We showed that music when it is humanized with Gaussian $1 / f^{\beta}$ noise is rated significantly better by listeners than the same sequences humanized with Gaussian white noise. We expect that in principle, after subtraction of accentuation, rhythmic long-range correlations exist when humans play on many different kinds of instruments, examples include musicians singing or playing the guitar or the piano.

Outlook It would be interesting to further develop a neurophysical model that accounts for time estimation on the time scale of milliseconds, where a starting point could be Davidsen's and Schuster's model [143]. Another open question is, in which way the correlation properties of the error time series change when two (or more) musicians play together. A simplified problem is, how the temporal 
correlations in a music rhythm played by a human are influenced by a metronome, which contains fluctuations around the exact metre. 


\section{Chapter 5}

\section{Conclusions and Outlook}

In conclusion we have studied transport phenomena, scale-free properties and fractal fluctuations in different complex dynamical systems. This thesis was presented in two main parts.

In the first part (Chap. 3), we have studied the dynamics of BECs in leaking optical lattices in the framework of the DNLS. We investigated the flux of ultracold atoms out of the optical trap and hence analyzed the survival probability $N(\tau)$ which decays in sudden jumps $J=\delta N$ for a certain range of the nonlinearity $\Lambda$. We found that these jumps, also referred to as avalanches, follow a scale-free distribution

$$
\mathcal{P}(J=\delta N) \sim 1 / J^{\alpha} .
$$

The avalanche events are accompanied by the formation of discrete breathers inside the optical lattice for a nonlinearity $\Lambda>\Lambda_{b}$. Our numerical findings indicate the existence of a phase transition at $\Lambda_{b}$. To this end, we proposed an order parameter which measures the relative number of sites that are occupied by the BEC. We have linked the observed power law distribution of jumps to the distribution of island sizes in the mixed phase space of the reduced system: the closed nonlinear trimer. Using the standard map as a paradigmatic model for mixed phase space dynamics, we provided evidence that a power law distribution of island sizes is a generic feature of the mixed phase space. In a simple model that imitates the selfsimilar hierarchical structure of the mixed phase space we found analytical bounds for the exponent yielding $1<\alpha<3$ in agreement with our numerical findings.

We explained the avalanche events using the scenario of a collision process involving a stationary DB and a lattice excitation (e.g. a moving breather) where a threshold exists, beyond which the DB gets destabilized. The destabilization of a DB due to the collision leads to a shift of the DB towards the perturbation. During the migration process, a fraction of the (atomic) density of the excitation 'tunnels' through the DB leaving the system as an avalanche. We showed that there exists a total energy threshold in the trimer, in order to enable a perturbation at site, say, 1 to trigger the destabilization of the DB at site 2. The destabilization threshold is described analytically by considering the dynamically accessible region of the phase space and linked to the Peierls-Nabarro barrier of a single DB. Furthermore, an upper boundary for the (atomic) density that tunnels through the DB to site 3 is given analytically. The existence of the destabilization threshold could be used as 
a tool for controlling the transmission of coherent atomic beams in interferometry and quantum information processes.

The DNLS can be applied to a variety of physical systems, a prominent example are coupled nonlinear optical waveguide arrays. In this context, avalanches of BECs translate into the scale-free statistics of light emittance from waveguide arrays. Discrete breathers in such two-dimensional networks were investigated in the past years both theoretically and experimentally and can exhibit a rich variety of functional operations such as blocking, routing or logic functions [4, 5]. Experimental evidence of the destabilization process of the stationary DB is expected to be observable in nonlinear waveguide arrays and might lead to functional operations such as filtering optical beams.

In the second part (Sec. 4.1) we analyzed the ballistic flux of electrons through mesoscopic systems such as quantum dots, nanowires and electron billiards. Motivated by unexpected experimental findings on fractal conductance fluctuations in electron billiards, where the fractal dimension changed with the coherence length, we simulated purely classically the conductance through electron billiards with different geometries and found clear numerical evidence for FCF of classical origin. We have shown using standard map, that transport through chaotic Hamiltonian systems in general produces fractal conductance curves, where the fractal dimension reflects the distribution of lobe widths $P(w) \sim w^{-\alpha}$ in the entry-/exitset which is the phase space projection of the leads attached to the conductor. We emphasize, that chaotic dynamics, through its fundamental property of stretching and folding in phase space, leads to such a lobe structure, which is typical for chaotic systems and not special to the standard map. An analytical estimation of the fractal dimension yields $D=\alpha-1 / 2$.

To investigate the origin of the power law distribution of the lobe widths, we examined the simplest case of an open chaotic area preserving map the dynamics of which is governed by a single, positive homogeneous Lyapunov exponent $\lambda$. We showed that under these conditions the number of lobes of width $w$ in the entryset reads

$$
P(w) \propto w^{\frac{1}{\lambda \tau}-1}
$$

with mean dwelltime $\tau$. This suggests that the power law distribution of lobe widths is a generic property even for fully chaotic systems.

It is known that the semiclassical theory leads to fractal conductance curves due to interference of the numerous electron paths. To connect our results with the semiclassical picture of FCF, note that an implicit assumption of the semiclassical theory is that the classical dynamics remains unchanged as the external parameter is varied and thus only phase changes are relevant. Here it was shown that the classical chaotic dynamics itself already leads to fractal conductance curves, i.e. even changes of the classical dynamics on arbitrary small scales lead to fluctuations of the conductance. Due to the fractal nature of the classical conductance, there is no parameter scale that separates coherent and incoherent fluctuations.

In conclusion we are led to the following scenario: In general the conductance curve of low-dimensional Hamiltonian systems is a superposition of two fractals one originating in interference which is suppressed by decoherence to reveal the 
fractal fluctuations reflecting the classical phase space structure. This might explain the unexpected results of experiments in semiconductor quantum dots where a dependence of the fractal dimension on the coherence length was observed. Furthermore, we predict fractal fluctuations in the conductance of low-dimensional Hamiltonian systems with a purely chaotic phase space.

In Sec. 4.2 we considered temporal fluctuations in music rhythms played by humans yielding long-range correlations. We showed by analyzing a set of different rhythmic tasks, that the temporal fluctuations in simple as well as in more complex music rhythms generically exhibit Gaussian $1 / f^{\beta}$ noise. A range of exponents $0.2<\beta<1.3$, depending on the individual and the task recorded, was found. The fact that long-memory processes arise in a broad variety of complex rhythmic tasks strongly supports the hypothesis, that a common basic neurophysical mechanism accounts for interval timing in the millisecond regime (see [140, 141] for an overview). We shortly reviewed an idea based on a simple stochastic model [143] that generates spike trains exhibiting long-range correlations $\left(1 / f^{\beta}\right.$-type), which is work in progress.

Using humanized music, we showed that the $1 / f^{\beta}$ humanized versions (with $\beta \approx 1$ ) were rated significantly better by listeners than the conventional humanized sequences comprising a Gaussian white noise error source. Applications are possible in humanizing music sequences with Gaussian $1 / f^{\beta}$ noise, e.g. in the field of electronic music or in post-production of real recordings. To this end, a patent in the EU and the USA was filed by the Max Planck Society, termed "Method and device for humanizing music sequences".

As an outlook on future works on the DNLS with leaking boundaries ("avalanches of BECs"), it would be fascinating to see the experimental verification of the predicted avalanche statistics. Second, even though we do have clear numerical evidence for the phase transition, which is related to the formation of DBs in the open discrete nonlinear system, the origin of the phase transition together with an analytical expression for $\Lambda_{b}$ is still an open and fascinating question (see [28, 77, 101]) and work in progress [87.

As far as the study on the closed nonlinear trimer and the observed mechanism of destabilization of a DB is concerned (Sec. 3.8), a natural next step is the analysis of the nonlinear trimer including dissipation. This may provide a better description of the transfer of atoms/energy through the DB and a link the results to experimental systems (e.g. a molecular trimer) might be possible. A promising theoretical approach could be the Holstein model. Another system of interest consists of three nonlinear sites embedded in a linear chain (see as well [106]) to enable investigations of collisions of DBs with linear lattice excitations.

On the outlook on FCF, it would be of high interest to study the transition from the semiclassical to the classical limit where experimentally a smooth change of the fractal dimension was observed. So far, rigorous theoretical descriptions exist only for the two limiting cases, i.e. in the classical case (analyzed as part of this thesis) and in the semiclassical limit [17, 19].

We hope that we contributed a small part in solving the puzzles emerging from scale-free fluctuations in complex systems. 


\section{Appendix A}

\section{Limiting Cases of the Participation Ratio}

We will calculate the participation ratio (Eq. 3.17)

$$
\mathcal{P R}(\tau)=\left\langle\frac{[N(\tau)]^{2}}{M \sum_{n}\left|\psi_{n}(\tau)\right|^{4}}\right\rangle
$$

(which is a measure of the relative number of sites occupied by atoms in the leaking OL) for the closed system (i.e. $\gamma=0$ ) in the two limiting cases $\Lambda \gg 1$ and $\Lambda=0$.

\section{(a) $\Lambda \gg 1$.}

In the strong nonlinearity regime, there are $\mathcal{O}(M)$ number of DBs and each site is effectively decoupled from other sites, so each site will retain the same density for all time. Since the initial condition is such that the distribution of density at each site follows a uniform distribution, at other times, one expects the density to follows a uniform distribution, i.e. $P\left(\psi_{n}\right)=\frac{1}{2 b}$ where we assume that the $\psi_{n}$ 's are drawn from a uniform distribution and $y$ runs from $-b$ to $+b$. Therefore,

$$
\sum_{n=1}^{M}\left|\psi_{n}\right|^{4}=M<\left|\psi_{n}\right|^{4}>_{n} \approx M<\left|\psi_{n}\right|^{4}>_{\psi_{n}}
$$

In the second step, we assume that taking an average over all sites is approximately the same as taking an average over all possible $\psi_{n}$ 's.

Let us turn the right-hand side of the above equation into an integral

$$
M<|\psi|^{4}>_{\psi}=M \int_{-b}^{b}|\psi|^{4} P(\psi) d(\psi)=\frac{M b^{4}}{5}
$$

The normalization condition for the number of particles reads

$$
\sum_{n=1}^{M}\left|\psi_{n}\right|^{2}=M<\left|\psi_{n}\right|^{2}>_{n} \approx M<|\psi|^{2}>_{\psi}=M \int_{-b}^{b} \psi^{2} P(\psi) d \psi=\frac{M b^{2}}{3}=1
$$

which leads to $b=\sqrt{3 / M}$. Thus, we obtain

$$
M \sum_{n=1}^{M}\left|\psi_{n}\right|^{4} \approx \frac{M^{2} b^{4}}{5}=\frac{9}{5}
$$


and the participation ratio is $5 / 9$.

(b) $\Lambda=0$.

In the linear regime $(\Lambda=0)$, we found that the distribution of atomic density follows an exponential distribution, corresponding to the fact that in a linear lattice it is exponentially less likely to find higher excitations. The normalization condition of the probability function $\int_{0}^{1} P(x) d x=1$ yields

$$
P(x)=M e^{-M x},
$$

where $x=\left|\psi_{n}\right|^{2}$. The normalization condition for the total number of atoms reads

$$
\sum_{n=1}^{M}\left|\psi_{n}\right|^{2}=M<\left|\psi_{n}\right|^{2}>_{n} \approx M<x>_{x}=M \int_{0}^{1} x P(x) d x=1
$$

To determine $\mathcal{P} \mathcal{R}$

$$
\begin{aligned}
\sum_{n=1}^{M}\left|\psi_{n}\right|^{4} \approx M<|\psi|^{4}>_{\psi} & =M^{2} \int_{0}^{1} x^{2} P(x) d x \\
& =M^{2} \int_{0}^{1} x^{2} M e^{-M x} d x \\
& =-M^{2} e^{-M}+2\left(M \int_{0}^{1} x P(x) d x\right) \\
& =-M^{2} e^{-M}+2,
\end{aligned}
$$

where we used Eq. A.6 in the last step. Hence, in the thermodynamic limit we have

$$
\lim _{M \rightarrow \infty} \sum_{n=1}^{M}\left|\psi_{n}\right|^{4}=2
$$

leading to $\mathcal{P} \mathcal{R}=\frac{1}{2}$. 


\section{Appendix B}

\section{Distribution of Islands (Mixed Phase Space)}

Here, we aim to verify the hypothesis that the sizes of islands of a typical Hamiltonian mixed phase space system follow a power-law distribution. This is done through numerical estimation of the distribution of island sizes in the standard map (in the mixed phase space regime).

We consider a region $\mathcal{R}$ (see rectangular region in Fig. 3.15) in the phase space of the standard map containing an island (with its daughter islands) originating from a period 1 resonance ${ }^{1}$. We start two trajectories at random initial conditions $\mathbf{r}$ and $\mathbf{r}+\mathbf{s}$ within $\mathcal{R}$ separated by a distance $s=|\mathbf{s}|$. We follow the trajectories up to time $T$ and proclaim a trajectory to be inside an island if it has not left the region $\mathcal{R}$, with less and less error with increasing $T$. Eventually, we want to take the limit of $T \rightarrow \infty$. The quantity we are going to study is

$p_{\text {in,out }}(s)=$ Probability that the first trajectory is inside an island and the second is not.

This probes the circumferences of the islands and will help us to determine the distribution of island sizes. Let $p_{I}(R)$ be the island size distribution that we are interested in, $A_{I}$ the total area of islands in $\mathcal{R}$ and $A$ the total area of $\mathcal{R}$. The probability of an arbitrarily chosen point to lie inside an island is $P($ in $)=A_{I} / A$, where $A_{I}=\int p_{I}(R) \pi R^{2} d R$. The probability for a point known to be regular to lie inside an island of linear dimension $R$ (e.g. maximum diameter) is given by

$$
P(R \mid \text { in })=\frac{p_{I}(R) \pi R^{2}}{A_{I}}
$$

Now let us start with a point $r_{0}$ that is inside an island of size $R$ (see Fig. B.1 a). It contributes to $P(R \mid$ in $)$ with $P\left(r_{0}, R \mid\right.$ in $)=p_{I}(R) / A_{I}$. What is the probability $P_{\text {in,out }}(s, R)$ that a point inside a neighborhood of radius $s$ around $r_{0}$ lies outside the island, given that $r_{0}$ lies inside an island of size $R$ ? In the following, we consider the two limiting cases $R \gg s$ and $R \ll s$.

1.) Case $R \gg s$. If we approximate the island boundary by a straight line, the red shared area indicated in Fig. B.1p is the area that we are interested in. Thus, for

\footnotetext{
${ }^{1}$ For a period $\mathrm{N}$ resonance, we could do the same with the $\mathrm{N}$-th iteration of the map.
} 


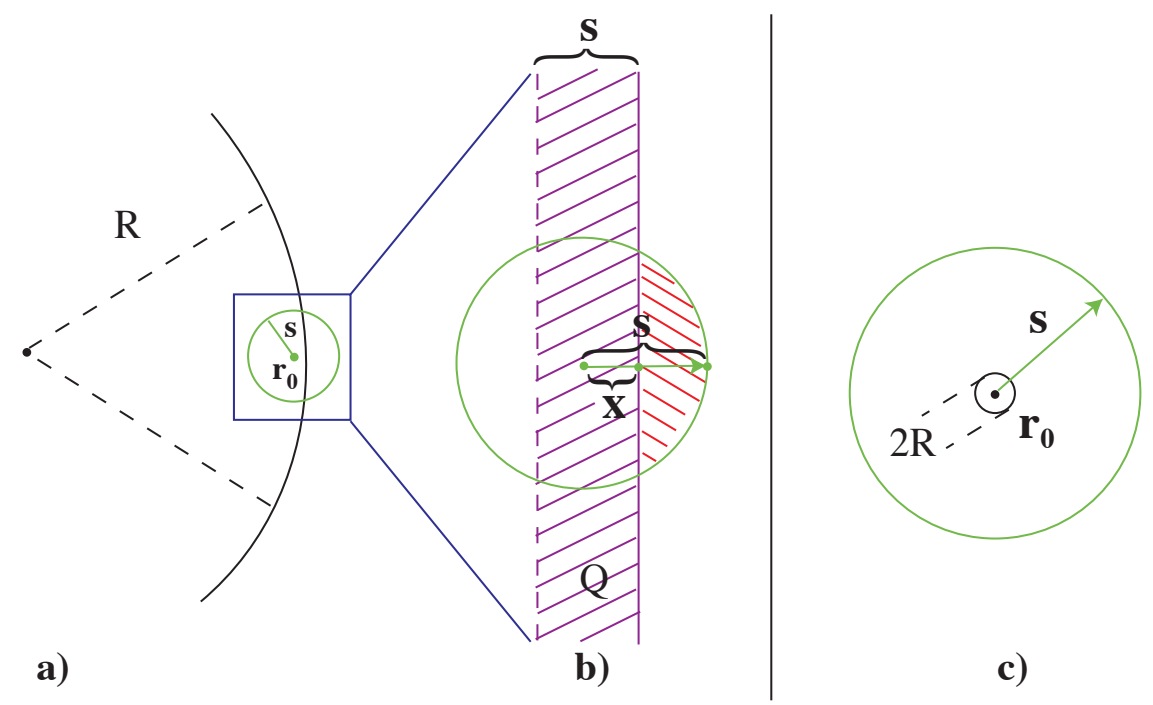

Figure B.1: Sketches for the two cases $R \gg s$ and $R \ll s$

a fixed $r_{0}$ in an island of size $R$, the fraction of area in its $s$-neighborhood that is outside the island is approximated by

$$
P\left(s, \text { out } \mid r_{0}, R\right)=\frac{1}{\pi s^{2}} \begin{cases}2 \int_{x}^{s} \sqrt{s^{2}-x^{\prime 2}} d x^{\prime} & \text { if } r_{0} \in Q \\ 0 & \text { else. }\end{cases}
$$

The only non-zero contributions come from the $r_{0}$ 's inside an island and are near the island boundary, i.e. $r_{0} \in Q$.We can thus write

$$
\begin{aligned}
P_{\text {in }, \text { out }}(s, R) & =\int_{\text {Island }} P\left(r_{0}, R \mid \text { in }\right) P\left(s, \text { out } \mid r_{0} R\right) d^{2} r_{0} \\
& =\frac{p_{I}(R)}{A_{I}} \int_{Q} \int_{x}^{s} \frac{2}{\pi s^{2}} \sqrt{s^{2}-x^{\prime 2}} d x^{\prime} d^{2} r_{0} \\
& =\frac{p_{I}(R)}{A_{I}} \frac{2}{\pi s^{2}} \int_{0}^{2 \pi R} \int_{0}^{s} \int_{x}^{s} \sqrt{s^{2}-x^{\prime 2}} d x^{\prime} d x d y \\
& =\frac{p_{I}(R)}{A_{I}} \frac{4 R}{s^{2}} \int_{0}^{s} \frac{1}{4}\left(\pi s^{2}-2 x \sqrt{s^{2}-x^{2}}-2 s^{2} \arctan \frac{x}{\sqrt{s^{2}-x^{2}}}\right) d x \\
& =\frac{p_{I}(R)}{A_{I}} \frac{4 R}{s^{2}} \frac{s^{3}}{3}=\frac{4}{3 A_{I}} R p_{I}(R) s \quad(\text { for } R \gg s) .
\end{aligned}
$$

2.) In the case $R \ll s$, we have (see Fig. B.1 c)

$$
P\left(s, \text { out } \mid r_{0}, R\right)=\frac{\pi s^{2}-\pi R^{2}}{\pi s^{2}} .
$$


And thus

$$
\begin{aligned}
P_{\text {in }, \text { out }}(s, R) & =\int_{\text {Island }} P\left(r_{0}, R \mid \text { in }\right) P\left(s, \text { out } \mid r_{0} R\right) d^{2} r_{0} \\
& =\frac{p_{I}(R)}{A_{I}} \times \frac{s^{2}-R^{2}}{s^{2}} \times \int_{\text {Island }} d^{2} r_{0} \\
& =\frac{\pi R^{2}}{A_{I}} p_{I}(R)\left(1-R^{2} / s^{2}\right)(\text { for } R \ll s) .
\end{aligned}
$$

In the numerics, we look at points on the perimeter of the s-neighborhood, thus we have the contributions from all island sizes, hence

$$
p_{\text {in }, \text { out }}(s)=\int_{0}^{R_{\max }} \frac{d}{d s} P_{\text {in }, \text { out }}(s, R) d R .
$$

Substituting

$$
\frac{d}{d s} P_{\text {in,out }}(s, R)= \begin{cases}\frac{4}{3 A_{I}} R p_{I}(R) & \text { if } s \ll R \\ \frac{2 \pi R^{4}}{A_{I}} p_{I}(R) \frac{1}{s^{3}} & \text { if } s \gg R\end{cases}
$$

and splitting the integral (thereby approximating the integral by its two limiting cases), we obtain

$$
p_{\text {in }, \text { out }}(s)=\int_{0}^{s} \frac{2 \pi R^{4}}{A_{I}} p_{I}(R) \frac{1}{s^{3}} d R+\int_{s}^{R_{\max }} \frac{4}{3 A_{I}} R p_{I}(R) d R .
$$

We further assume that $p_{I}(R)=\beta R^{-\alpha}$ (with $\alpha<3$ ), where $\alpha$ is the exponent which we will measure numerically in the mixed phase space of the standard map. Hence, the probability $p_{\text {in,out }}(s)$ reads

$$
\begin{aligned}
p_{\text {in }, \text { out }}(s) & =\frac{2 \pi \beta}{A_{I} s^{3}} \int_{0}^{s} R^{4-\alpha} d R+\frac{4 \beta}{3 A_{I}} \int_{s}^{R_{\max }} R^{1-\alpha} d R \\
& =\frac{2 \pi \beta}{A_{I} s^{3}} \frac{s^{5-\alpha}}{5-\alpha}+\frac{4 \beta}{3 A_{I}(2-\alpha)}\left[R_{\max }^{2-\alpha}-s^{2-\alpha}\right],
\end{aligned}
$$

and can thus be written in the form $p_{\text {in,out }}(s)=C_{0}+C_{1} s^{2-\alpha}$ as stated in Eq. 3.27. 


\section{Appendix C}

\section{Questionnaire and Lyrics}

\section{Report on questionnaire}

This questionnaire aims at investigating how certain types of rhythmic drum sequences are rated by humans. There are three different versions of drum sequences under investigation, which differ only by their error time series Eq. 4.13 . The error time series are of the three types:

1. Version E ('exact'): $e_{n}=0 \forall n$.

2. Version C ('correlated'), comprising Gaussian $1 / f$ noise with exponent $\beta \approx 1$.

3. Version W ('white'): $e_{n}=\xi_{n}$, where $\xi_{n}$ is Gaussian white noise.

To compare the versions $\mathrm{E}, \mathrm{C}$ and $\mathrm{W}$, we choose the same standard deviations $\sigma_{C}=\sigma_{W}$ of the error time series and mean $<e_{E}>=<e_{C}>=<e_{W}>=0$. In the experiment 16 test subjects from the Göttingen Symphonic Orchestra (GSO) of mean age $36.5 \pm 12.3$ participated. The experiment consists of two parts $\mathrm{A}$ and $\mathrm{B}$, defined by sequences with error time series with either $\sigma_{A}=6 \mathrm{~ms}$ or $\sigma_{B}=10$ ms. In the following, the $\chi^{2}$ method is used. The level of significance is $p=0.05$. The main results are:

- The test subjects from the GSO could distinguish, if two sequences are different or the same for both cases $\sigma=6 \mathrm{~ms}$ and for $\sigma=10 \mathrm{~ms}$, if compared to a random choice with $50 \%$ probability.

- For $\sigma=10 \mathrm{~ms}$ the version $\mathrm{E}$ was rated significantly better than both $\mathrm{C}$ and $\mathrm{W}$. The version $\mathrm{C}$ was rated better than W. For $\sigma=6 \mathrm{~ms}$ the version $\mathrm{E}$ was rated significantly better than W. By comparing the versions E-C and C-W no preference was found.

We used the following questionnaire design. The whole experiment consists of 5 pairs of sequences in part A $(\sigma=6 \mathrm{~ms})$ and another 5 pairs of sequences in part $\mathrm{B}(\sigma=10 \mathrm{~ms})$. In each part, there are two pairs of identical and three pairs of different sequences. The computer-generated rhythms in the two parts $\mathrm{A}$ and $\mathrm{B}$ are different. Two sequences (also referred to as samples) were played to the test subjects, to whom it was told that they shall evaluate drum samples and that the 
order of samples is random, which means that one or more pairs of samples may as well be identical. Then, there were two main questions given to the test subjects.

1. Are the two samples different?

2. Which sample do you like better?

In order to eliminate unwanted effects (e.g. that the first sample of a pair may in average be rated better than the second or vice versa), the samples were arranged using specific symmetries. Therefore, the test subjects were divided into four groups. Given a random order $\left(a_{i}, b_{i}\right), i=1 \ldots 5$, of integer pairs in group 1, then the order for the other groups is as follows. Samples in group 2 are reversely ordered, in group 3 the order of each pair is switched and group 4 consists of reversely ordered switched pairs of samples, leading to pairs $\left(b_{5-i}, a_{5-i}\right),\left(b_{i}, a_{i}\right)$ and $\left(a_{5-i}, b_{5-i}\right)$ respectively.

Analysis of hypotheses. Null hypothesis 1: "The decision, if two samples are the same or not, was by chance (with probability 0.5 )." The null hypothesis 1 is rejected for both $\sigma_{A}=6 \mathrm{~ms}$ and $\sigma_{B}=10 \mathrm{~ms}$. For samples with $\sigma_{A}$ there were $N_{A}=79$ answers given, thereof 53 correct (67\%), significance $p_{A}=0.002$, $\chi^{2}=9.23$, and for samples with $\sigma_{B}: N_{B}=79$ answers, thereof 50 correct $(63 \%)$, $p_{B}=0.02, \chi^{2}=5.58$.

Null hypothesis 2: "The samples sound equally good." For samples with $\sigma_{A}=10$ $\mathrm{ms}$ the results are given in Fig. 4.26. For versions with $\sigma_{B}=6 \mathrm{~ms}$ the results of the experiments are

- Comparison E-W: There were 10 answers given, thereof 10 in favor of version E, significance $p=0.002, \chi^{2}=10.0$. Null hypothesis 2 is rejected (i.e. E was rated better than $\mathrm{W})$.

- Comparison C-W and E-C: The null hypothesis 2 can not be rejected, i.e. no significant preference was found.

It seems like the auditory threshold for the task of qualitatively differentiating between versions that vary in the time domain is reached with difficulty level $\sigma_{A}=6$ ms. It is evident that humans are able to differ between signals of much shorter time difference, if the signals are played at the same time. However, the versions in this experiment were played one after another and not on top of each other, which, of course, makes the task of differencing for test subjects more difficult.

\section{Lyrics}

In the following we will provide additional information and the lyrics for the $1 / f$ humanized song, that was generated in the course of this thesis. The song is of length 4:05 min and courtesy of Max Planck Institute for Dynamics and SelfOrganization. The term chaos theory entered the second line of the lyrics shown in Fig. C.1.

- Title: Everyday, Everynight 


\begin{tabular}{|l|l|}
\hline $\begin{array}{l}\text { 1. (Verse 1) } \\
\text { One day you came and made me see } \\
\text { my chaos got no theory } \\
\text { but love - you can't explain } \\
\text { I didn't know what I would miss } \\
\text { but then you gave me your first kiss } \\
\text { I feel -- I go insane }\end{array}$ & $\begin{array}{l}\text { 5. (Bridge) } \\
\text { You light my way } \\
\text { day by day } \\
\text { and when you stay } \\
\text { you'll take my breath away }\end{array}$ \\
\hline $\begin{array}{l}\text { 2. (Bridge) } \\
\text { You light my way } \\
\text { day by day } \\
\text { and when you stay } \\
\text { you'll take my breath away }\end{array}$ & $\begin{array}{l}\text { 6. (Chorus) } \\
\text { Every day - I want you by my side } \\
\text { and every day - just me and you } \\
\text { every night - You are my shining light } \\
\text { and every night - my dreams come true }\end{array}$ \\
\hline $\begin{array}{l}\text { 3. (Chorus) } \\
\text { Every day } \\
\text { I want you by my side } \\
\text { and every day } \\
\text { just me and you }\end{array}$ & $\begin{array}{l}\text { 7. („C-part“) } \\
\text { When the world is cold outside } \\
\text { you give me love and hold me tight } \\
\text { you'll be right there in every way } \\
\text { every day }\end{array}$ \\
\hline $\begin{array}{l}\text { Y. (Verse 2) } \\
\text { You came closer, it was physical } \\
\text { but attraction is not logical } \\
\text { I wanna see you look at me } \\
\text { cause this will be my memory } \\
\text { when we are old and grey }\end{array}$ & $\begin{array}{l}\text { 8. (Chorus) } \\
\end{array}$ \\
\hline
\end{tabular}

Figure C.1: Lyrics for the song "Everyday, everynight".

- Lyrics: Nadine Zacharias

- Music and mixing: Cubeaudio Recording Studio 


\section{Appendix D}

\section{Audio Examples}

Supplementary material: CD with audio examples of humanized music pieces ${ }^{1}$. For each piece of music three version were generated, that differ only in the rhythmic structure (see Sec. 4.2):

- E - exact version,

- C - 1/f humanized version,

- W - white noise humanized version.

Examples (1-3) are simple computer-generated drum rhythms of approx. $30 \mathrm{sec}$ length each.

Audio example 1: Version E

Audio example 2: Version $C$

Audio example 3: Version $W$

Examples (4-6) are samples of the humanized pop song "Everyday, everynight" created and recorded in collaboration with Cubeadio recording studio.

Audio example 4: Version $C, 5$ sec pause, version $E$

Audio example 5: Versions $W, 5$ sec pause, version $C$

Audio example 6: Version $C$ (complete song, 4:05 min)

Standard deviation used for humanizing is $\sigma=15 \mathrm{~ms}$ for examples (1-5) and $\sigma=7.5 \mathrm{~ms}$ for example 6 .

\footnotetext{
${ }^{1}$ Electronic version of this thesis: Audio files are attached.
} 


\section{Bibliography}

[1] D Hennig and GP Tsironis. Wave transmission in nonlinear lattices. Physics Reports 307, 333 (1999).

[2] T Kottos and M Weiss. Current relaxation in nonlinear random media. Phys. Rev. Lett. 93, 190604 (2004).

[3] Y S Kivshar. Self-localization in arrays of defocusing waveguides. Opt. Lett. 18, 1147 (1993).

[4] D Christodoulides and E Eugenieva. Blocking and routing discrete solitons in two-dimensional networks of nonlinear waveguide arrays. Phys. Rev. Lett. 87, 233901 (2001).

[5] DN Christodoulides, F Lederer, and Y Silberberg. Discretizing light behaviour in linear and nonlinear waveguide lattices. Nature 424, 817-823 (2003).

[6] M Greiner, O Mandel, T Esslinger, TW Hänsch, and I Bloch. Quantum phase transition from a superfluid to a mott insulator in an ultracold gas of atoms. Nature 415, 39 (2002).

[7] FS Cataliotti, S Burger, C Fort, P Maddaloni, F Minardi, A Trombettoni, A Smerzi, and M Inguscio. Josephson junction arrays with bose-einstein condensates. Science 293, 843-846 (2001).

[8] II Carusotto and GC La Rocca. Modulated optical lattice as an atomic fabryperot interferometer. Phys. Rev. Lett. 84, 399 (2000).

[9] J Lye, L Fallani, M Modugno, D Wiersma, and C Fort. Bose-einstein condensate in a random potential. Phys. Rev. Lett. 95, 070401 (2005).

[10] C Fort, L Fallani, V Guarrera, JE Lye, M Modugno, DS Wiersma, and M Inguscio. Effect of optical disorder and single defects on the expansion of a bose-einstein condensate in a one-dimensional waveguide. Phys. Rev. Lett. 95, 170410 (2005).

[11] T Schulte, S Drenkelforth, J Kruse, and W Ertmer. Routes towards andersonlike localization of bose-einstein condensates in disordered optical lattices. Phys. Rev. Lett. 95, 170411 (2005).

[12] D Clément, A Varón, M Hugbart, and J Retter. Suppression of transport of an interacting elongated bose-einstein condensate in a random potential. Phys. Rev. Lett. 95, 170409 (2005). 
[13] B Damski, J Zakrzewski, L Santos, P Zoller, and M Lewenstein. Atomic bose and anderson glasses in optical lattices. Phys. Rev. Lett. 91, 80403-80403 (2003).

[14] R Webb and S Washburn. Quantum interference fluctuations in disordered metals. Phys. Today 41, 46 (1988).

[15] C Marcus, A Rimberg, R Westervelt, and P Hopkins. Conductance fluctuations and chaotic scattering in ballistic microstructures. Phys. Rev. Lett. 69, 506 (1992).

[16] C Marcus, R Westervelt, P Hopkins, and A Gossard. Conductance fluctuations and quantum chaotic scattering in semiconductor microstructures. Chaos 3, 643 (1993).

[17] R Ketzmerick. Fractal conductance fluctuations in generic chaotic cavities. Phys. Rev. B 54, 10841 (1996).

[18] L Hufnagel, R Ketzmerick, and M Weiss. Conductance fluctuations of generic billiards: Fractal or isolated? Europhysics Letters 54, 703-708 (2001).

[19] A Sachrajda, R Ketzmerick, C Gould, and Y Feng. Fractal conductance fluctuations in a soft-wall stadium and a sinai billiard. Phys. Rev. Lett. 80, 1948 (1998).

[20] H Hegger, B Huckestein, K Hecker, M Janssen, A Freimuth, G Reckziegel, and R Tuzinski. Fractal conductance fluctuations in gold nanowires. Phys. Rev. Lett. 77, 3885-3888 (1996).

[21] A Micolich, R Taylor, R Newbury, and J Bird. Geometry-induced fractal behaviour in a semiconductor billiard. J. Phys.: Condens. Matter 10, 1339 (1998).

[22] Y Ochiai, L Lin, K Yamamoto, and K Ishibashi. Low-temperature magnetotransport in ballistic quantum dots and wires. Semicond. Sci. Technol. 13, A15 (1998).

[23] Y Takagaki, M ElHassan, A Shailos, and C Prasad. Magnetic-field-controlled electron dynamics in quantum cavities. Phys. Rev. B 62, 10255 (2000).

[24] Italo Guarneri and Marcello Terraneo. Fractal fluctuations in quantum integrable scattering. Phys. Rev. E 65, 4 (2001).

[25] F Pinheiro and C Lewenkopf. Fractal conductance fluctuations in electron billiards: a random matrix theory approach. Brazilian Journal of Physics 36, 379 (2006).

[26] R Voss and J Clarke. '1/f noise' in music and speech. Nature 258, 317 (1975).

[27] R Voss and J Clarke. "1/f noise" in music: Music from $1 / f$ noise. J. Acoust. Soc. Am. 63, 258 (1978). 
[28] G S Ng, H Hennig, R Fleischmann, T Kottos, and T Geisel. Avalanches of bose-einstein condensates in leaking optical lattices. New J. Phys. In press (2009).

[29] H Hennig, J Dorignac, and D K Campbell. In preparation (2009).

[30] H Hennig, R Fleischmann, L Hufnagel, and T Geisel. Fractal conductance fluctuations of classical origin. Phys. Rev. E 76, 015202(R) (2007).

[31] H Hennig, A Fredebohm, R Fleischmann, Y Hagmayer, Fabias Theis, A Witt, and T Geisel. In preparation (2009).

[32] A Fredebohm, H Hennig, Y Hagmayer, and R Fleischmann. In preparation (2009).

[33] Yoseph Imry. Introduction to mesoscopic physics. Oxford University Press (2002).

[34] LL Sohn. Nanotechnology a quantum leap for electronics. Nature 394, 131132 (1998).

[35] A J Lichtenberg and M A Lieberman. Regular and chaotic dynamics. Springer (1992).

[36] T Geisel, A Zacherl, and G Radons. Generic 1/f noise in chaotic hamiltonian dynamics. Phys. Rev. Lett. 59, 2503 (1987).

[37] T Geisel, A Zacherl, and G Radons. Chaotic diffusion and 1/f-noise of particles in two-dimensional solids. Z. Phys. B 71, 117-127 (1988).

[38] S Strogatz. Nonlinear dynamics and chaos. Westview Press (1994).

[39] B Chirikov. A universal instability of many-dimensional oscillator systems. Physics Reports 52, 263 (1979).

[40] L Reichl. The transition to chaos: Conservative classical systems and quantum manifestations. Springer (2004).

[41] FL Moore, JC Robinson, CF Bharucha, B Sundaram, and MG Raizen. Atom optics realization of the quantum kicked rotor. Phys. Rev. Lett. 75, 4598-4601 (1995).

[42] Paul Meakin. Fractals, scaling and growth far from equilibrium. Cambridge University Press (1998).

[43] B Dubuc, JF Quiniou, C Roques-Carmes, C Tricot, and SW Zucker. Evaluating the fractal dimension of profiles. Phys Rev A 39, 1500 (1989).

[44] M Taqqu, V Teverovsky, and W Willinger. Estimators for long-range dependence: An empirical study. FRACTALS 3, 785 (1995). 
[45] C Peng, S Havlin, H Stanley, and A Goldberger. Quantification of scaling exponents and crossover phenomena in nonstationary heartbeat time series. Chaos (1995).

[46] D Campbell, S Flach, and Y Kivshar. Localizing energy through nonlinearity and discreteness. Phys. Today (2004).

[47] DK Campbell. Fresh breather. Nature 432, 455-456 (2004).

[48] S Flach and AV Gorbach. Discrete breathers - advances in theory and applications. Physics Reports 467, 1 (2008).

[49] S Flach and C Willis. Discrete breathers. Physics Reports 295, 181 (1998).

[50] A S Dolgov. Localization of vibrations in a nonlinear crystalline structure. Sov. Phys. Solid State 28, 907 (1986).

[51] A Sievers and S Takeno. Intrinsic localized modes in anharmonic crystals. Phys. Rev. Lett. 61, 970 (1988).

[52] E Trías, J Mazo, and T Orlando. Discrete breathers in nonlinear lattices: Experimental detection in a josephson array. Phys. Rev. Lett. 84, 741 (2000).

[53] M Sato, B. E Hubbard, A. J Sievers, B Ilic, and H. G Craighead. Optical manipulation of intrinsic localized vibrational energy in cantilever arrays. Europhys. Lett. 66, 318 (2007).

[54] Jason W Fleischer, Mordechai Segev, Nikolaos K Efremidis, and Demetrios N Christodoulides. Observation of two-dimensional discrete solitons in optically induced nonlinear photonic lattices. Nature 422, 147-150 (2003).

[55] HS Eisenberg, Y Silberberg, R Morandotti, AR Boyd, and JS Aitchison. Discrete spatial optical solitons in waveguide arrays. Phys. Rev. Lett. 81, 3383 (1998).

[56] A Xie, L van der Meer, W Hoff, and R Austin. Long-lived amide i vibrational modes in myoglobin. Phys. Rev. Lett. 84, 5435 (2000).

[57] M Sato and A J Sievers. Direct observation of the discrete character of intrinsic localized modes in an antiferromagnet. Nature 432, 486 (2004).

[58] Roberto Livi, Roberto Franzosi, and Gian-Luca Oppo. Self-localization of bose-einstein condensates in optical lattices via boundary dissipation. Phys. Rev. Lett. 97, 4 (2006).

[59] E Fermi, J Pasta, and S Ulam. Studies of nonlinear problems. LASL Report LA-1940 (1955).

[60] YS Kivshar and DK Campbell. Peierls-nabarro potential barrier for highly localized nonlinear modes. Phys. Rev. E 48, 3077-3081 (1993). 
[61] Benno Rumpf. Intermittent movement of localized excitations of a nonlinear lattice. Phys. Rev. E 70, 9 (2004).

[62] GP Tsironis. If "discrete breathers" is the answer, what is the question? Chaos 13, 657 (2003).

[63] BP Anderson and MA Kasevich. Macroscopic quantum interference from atomic tunnel arrays. Science 282, 1686 (1998).

[64] D Jaksch, C Bruder, JI Cirac, CW Gardiner, and P Zoller. Cold bosonic atoms in optical lattices. Phys. Rev. Lett. 81, 3108 (1998).

[65] C Orzel, A Tuchman, M Fenselau, and M Yasuda. Squeezed states in a bose-einstein condensate. Science 291, 2386 (2001).

[66] I Bloch. Ultracold quantum gases in optical lattices. Nat. Phys. 1, 23-30 (2005).

[67] B Swanson, J Brozik, S Love, and G Strouse. Observation of intrinsically localized modes in a discrete low-dimensional material. Phys. Rev. Lett. 82, 3288 (1999).

[68] N Voulgarakis, G Kalosakas, and A Bishop. Multiquanta breather model for ptcl. Phys. Rev. B 64, 020301 (2001).

[69] K Kladko, J Malek, and AR Bishop. Intrinsic localized modes in the chargetransfer solid ptcl. J. Phys.: Condens. Matter 11, 415-422 (1999).

[70] U Schwarz, L English, and A Sievers. Experimental generation and observation of intrinsic localized spin wave modes in an antiferromagnet. Phys. Rev. Lett. 83, 223 (1999).

[71] L Floría, J Marín, P Martínez, F Falo, S Aubry, LL Brillouin, and CE Saclay. Intrinsic localisation in the dynamics of a josephson-junction ladder. Europhys. Lett 36, 539 (1996).

[72] A Ustinov. Imaging of discrete breathers. Chaos 13, 716 (2003).

[73] M Sato, B Hubbard, A Sievers, and B Ilic. Observation of locked intrinsic localized vibrational modes in a micromechanical oscillator array. Phys. Rev. Lett. 90, 044102 (2003).

[74] R Morandotti, U Peschel, and J Aitchison. Dynamics of discrete solitons in optical waveguide arrays. Phys. Rev. Lett. 83, 2726 (1999).

[75] J Dorignac, JC Eilbeck, M Salerno, and AC Scott. Quantum signatures of breather-breather interactions. Phys. Rev. Lett. 93, 25504-25504 (2004).

[76] AR Bishop, G Kalosakas, KØ Rasmussen, and PG Kevrekidis. Localization in physical systems described by discrete nonlinear schrödinger-type equations. Chaos 13, 588 (2003). 
[77] P Kevrekidis, J Espinolarocha, Y Drossinos, and A Stefanov. Dynamical barrier for the formation of solitary waves in discrete lattices. Physics Letters A 372, 2247-2253 (2008).

[78] S Aubry. Breathers in nonlinear lattices: existence, linear stability and quantization. Physica D: Nonlinear Phenomena 103, 201-250 (1997).

[79] R Mackay and S Aubry. Proof of existence of breathers for time-reversible or hamiltonian networks of weakly coupled oscillators. Nonlinearity 7, 1623 (1994).

[80] G Tsironis and S Aubry. Slow relaxation phenomena induced by breathers in nonlinear lattices. Phys. Rev. Lett. 77, 5225 (1996).

[81] A Bikaki, NK Voulgarakis, S Aubry, and GP Tsironis. Energy relaxation in discrete nonlinear lattices. Phys. Rev. E 59, 1234 (1999).

[82] F Piazza, S Lepri, and R Livi. Slow energy relaxation and localization in $1 d$ lattices. J. Phys. A 34, 9803 (2001).

[83] F Piazza, S Lepri, and R Livi. Cooling nonlinear lattices toward energy localization. Chaos 13, 637 (2003).

[84] M Eleftheriou, S Lepri, R Livi, and F Piazza. Stretched-exponential relaxation in arrays of coupled rotators. Physica D: Nonlinear Phenomena 204, 230-239 (2005).

[85] B Li and J Wang. Anomalous heat conduction and anomalous diffusion in one-dimensional systems. Phys. Rev. Lett. 91, 044301 (2003).

[86] Roberto Franzosi, Roberto Livi, and Gian-Luca Oppo. Probing the dynamics of bose-einstein condensates via boundary dissipation. J. Phys. B 40, 11951210 (2007).

[87] H Hennig, R Fleischmann, and T Geisel. Work in progress (2009).

[88] DN Christodoulides and RI Joseph. Discrete self-focusing in nonlinear arrays of coupled waveguides. Opt. Lett. 13, 794 (1988).

[89] DN Christodoulides and RI Joseph. Slow bragg solitons in nonlinear periodic structures. J. Appl. Phys Phys Rev Lett 62, 1746 (1989).

[90] R Khomeriki. Nonlinear band gap transmission in optical waveguide arrays. Phys. Rev. Lett. 92, 51 (2004).

[91] J Meier, G Stegeman, Y Silberberg, and R Morandotti. Nonlinear optical beam interactions in waveguide arrays. Phys. Rev. Lett. 93, 093903 (2004).

[92] H Martin, E Eugenieva, Z Chen, and D Christodoulides. Discrete solitons and soliton-induced dislocations in partially coherent photonic lattices. Phys. Rev. Lett. 92, 123902 (2004). 
[93] I Bloch, TW Hänsch, and T Esslinger. An atom laser with a cw output coupler. Phys. Rev. Lett. 82, 3008 (1999).

[94] A Öttl, Stephan Ritter, M Köhl, and Tilman Esslinger. Correlations and counting statistics of an atom laser. Phys. Rev. Lett. 95, 4 (2005).

[95] Immanuel Bloch and Wilhelm Zwerger. Many-body physics with ultracold gases. Rev. Mod. Phys. 80, 885-964 (2008).

[96] A Scott. Nonlinear science: Emergence and dynamics of coherent structures. Oxford University Press (2003).

[97] A Trombettoni and A Smerzi. Discrete solitons and breathers with dilute bose-einstein condensates. Phys. Rev. Lett. 86, 2353 (2001).

[98] G Moy, J Hope, and C Savage. Born and markov approximations for atom lasers. Phys. Rev. A 59, 667 (1999).

[99] J Jeffers, P Horak, SM Barnett, C Baxter, and P Radmore. Bound mode of an atom laser. Phys. Rev. A 62, 043602 (2000).

[100] H Jeldtoft Jensen. Self-organized criticality. Cambridge University Press (1998).

[101] F Palmero, R Carretero-González, P. G Kevrekidis, and W Królikowski. Solitons in one-dimensional nonlinear schrödinger lattices with a local inhomogeneity. Phys. Rev. E 77, 11 (2008).

[102] S Flach and CR Willis. Localized excitations in a discrete klein-gordon system. Phys. Lett. A 181, 232 (1993).

[103] S Mossmann and C Jung. Semiclassical approach to bose-einstein condensates in a triple well potential. Phys. Rev. A 74, 033601 (2006).

[104] M Ivanchenko, O Kanakov, V Shalfeev, and S Flach. Discrete breathers in transient processes and thermal equilibrium. Physica D: Nonlinear Phenomena 198, 120-135 (2004).

[105] Benno Rumpf. Simple statistical explanation for the localization of energy in nonlinear lattices with two conserved quantities. Phys. Rev. E 69, 5 (2004).

[106] R Vicencio, J Brand, and S Flach. Fano blockade by a bose-einstein condensate in an optical lattice. Phys. Rev. Lett. 98, 184102 (2007).

[107] G Kopidakis, S Aubry, and G Tsironis. Targeted energy transfer through discrete breathers in nonlinear systems. Phys. Rev. Lett. 87, 165501 (2001).

[108] S Datta. Electronic Transport in Mesoscopic Systems. (1995).

[109] D K Ferry and S M Goodnick. Transport in Nanostructures. (2005). 
[110] Rodolfo A Jalabert, Harold U Baranger, and A Douglas Stone. Conductance fluctuations in the ballistic regime: A probe of quantum chaos? Phys. Rev. Lett. 65, 2442-2445 (1990).

[111] HU Baranger, RA Jalabert, and AD Stone. Quantum-chaotic scattering effects in semiconductor microstructures. Chaos 3, 665 (1993).

[112] R Blümel and U Smilansky. Classical irregular scattering and its quantummechanical implications. Phys. Rev. Lett. 60, 477-480 (1988).

[113] R Fleischmann, T Geisel, and R Ketzmerick. Magnetoresistance due to chaos and nonlinear resonances in lateral surface superlattices. Phys. Rev. Lett. 68, 1367-1370 (1992).

[114] Y Lai, R Blümel, E OTT, and C Grebogi. Quantum manifestations of chaotic scattering. Phys. Rev. Lett. 68, 3491 (1992).

[115] JD Meiss and E OTT. Markov tree model of transport in area preserving maps. Physica 20D, 387 (1986).

[116] A Micolich, R Taylor, A Davies, and J Bird. Evolution of fractal patterns during a classical-quantum transition. Phys. Rev. Lett. 87, 036802 (2001).

[117] A Micolich, R Taylor, R Newbury, and J Bird. Discrete energy level spectrum dependence of fractal conductance fluctuations in semiconductor billiards. Physica E 13, 683 (2002).

[118] S Fishman, D Grempel, and R Prange. Chaos, quantum recurrences, and anderson localization. Phys. Rev. Lett. 49, 509 (1982).

[119] A Altland and MR Zirnbauer. Field theory of the quantum kicked rotor. Phys. Rev. Lett. 77, 4536-4539 (1996).

[120] P Jacquod and EV Sukhorukov. Breakdown of universality in quantum chaotic transport: the two-phase dynamical fluid model. Phys. Rev. Lett. 92, 116801 (2004).

[121] Y Chen, M Ding, and JA Scott Kelso. Long memory processes ( $1 / f$ type) in human coordination. Phys. Rev. Lett. 79, 4501-4504 (1997).

[122] DL Gilden, T Thornton, and MW Mallon. 1/f noise in human cognition. Science 267, 1837 (1995).

[123] S Roberts, R Eykholt, and M Thaut. Analysis of correlations and search for evidence of deterministic chaos in rhythmic motor control. Phys. Rev. E 62, 2597 (2000).

[124] Kjerstin Torre and Didier Delignières. Unraveling the finding of $1 / f$ noise in self-paced and synchronized tapping: a unifying mechanistic model. Biol. Cybern. 99, 159-170 (2008). 
[125] C Peng, S Buldyrev, A Goldberger, and S Havlin. Long-range correlations in nucleotide sequences. Nature 356, 168 (1992).

[126] T Geisel, J Nierwetberg, and A Zacherl. Accelerated diffusion in josephson junctions and related chaotic systems. Phys. Rev. Lett. 54, 616-619 (1985).

[127] Sh Kogan. Electronic noise and fluctuations in solids. Cambridge University Press (2008).

[128] R Mantegna and HE Stanley. An introduction to econophysics. Cambridge University Press (2000).

[129] JM Hausdorff, CK Peng, Z Ladin, JY Wei, and AL Goldberger. Is walking a random walk? evidence for long-range correlations in stride interval of human gait. Journal of Applied Physiology 78, 349-358 (1995).

[130] JL Cabrera and JG Milton. On-off intermittency in a human balancing task. Phys. Rev. Lett. 89, 158702-158702 (2002).

[131] I McHardy and B Czerny. Fractal x-ray time variability and spectral invariance of the seyfert galaxy ngc 5506. Nature 325, 696 (1987).

[132] A Lawrence, M Watson, K Pounds, and M Elvis. Low-frequency divergent x-ray variability in the seyfert galaxy ngc 4051. Nature 325, 694 (1987).

[133] M Teich. Fractal character of the auditory neural spike train. IEEE transactions on Biomedical Engineering 36, 150 (1989).

[134] Yuguo Yu, Richard Romero, and Tai Sing Lee. Preference of sensory neural coding for $1 / f$ signals. Phys. Rev. Lett. 94, 108103 (2005).

[135] I Procaccia and H Schuster. Functional renormalization-group theory of universal $1 / f$ noise in dynamical systems. Phys. Rev. A 28, 1210 (1983).

[136] M Kobayashi and T Musha. 1/f fluctuation of heartbeat period. IEEE transactions on Biomedical Engineering , 456-457 (1982).

[137] PC Ivanov, LA Amaral, A Goldberger, S Havlin, M Rosenblum, Z Struzik, and H Stanley. Multifractality in human heartbeat dynamics. Nature 399, 461 (1999).

[138] A L Goldberger. Fractal dynamics in physiology: Alterations with disease and aging. Proceedings of the National Academy of Sciences 99, 2466-2472 (2002).

[139] H Jennings, P Ivanov, A Martins, P Dasilva, and G Viswanathan. Variance fluctuations in nonstationary time series: a comparative study of music genres. Physica A: Statistical and Theoretical Physics 336, 585-594 (2004).

[140] DV Buonomano and UR Karmarkar. How do we tell time? The Neuroscientist 8, 42 (2002). 
[141] CV Buhusi and WH Meck. What makes us tick? functional and neural mechanisms of interval timing. Nature Reviews Neuroscience 6, 755-765 (2005).

[142] T Rammsayer and R Ulich. Counting models of temporal discrimination. Psychonomic Bulletin \& Review 8, 270 (2001).

[143] Jörn Davidsen and Heinz Georg Schuster. Simple model for $1 / f$ noise. Phys. Rev. E 65, 4 (2002).

[144] JP Bello, L Daudet, S Abdallah, C Duxbury, M Davies, and MB Sandler. A tutorial on onset detection in music signals. IEEE Transactions on Speech and Audio Processing 13, 1035-1047 (2005).

[145] JP Bello, C Duxbury, M Davies, and M Sandler. On the use of phase and energy for musical onset detection in the complex domain. IEEE Signal Processing Letters 11, 553-556 (2004).

[146] Jan Beran. Statistics for long-memory processes. Chapman \& Hall/CRC (1994).

[147] J Aldrich. R.a. fisher and the making of maximum likelihood 1912-1922. Statistical Science 12, 162 (1997).

[148] B Pilgram and D Kaplan. A comparison of estimators for $1 / f$ noise. Physica D 114, 108 (1998).

[149] A L Goldberger, L Amaral, L Glass, J Hausdorff, PC Ivanov, RG Mark, JE Mietus, G B Moody, C Peng, and HE Stanley. Physiobank, physiotoolkit, and physionet. Circulation 101, 215 (2000).

[150] L Glass and M C Mackey. From clocks to chaos. Princeton University Press (1988).

[151] WM Joiner and M Shelhamer. A model of time estimation and error feedback in predictive timing behavior. J. Comput. Neurosci. 26, 119 (2008).

[152] R Memmesheimer and M Timme. Non-additive coupling enables stable propagation of synchronous spiking in purely random networks. BMC Neuroscience (2007). 


\section{List of Abbreviations}

BEC - Bose-Einstein condensation (also: Bose-Einstein condensate)

DB - Discrete breather

DFA - Detrended fluctuation analysis

DNLS - Discrete nonlinear Schrödinger equation

FCF - Fractal conductance fluctuations

LRC - Long-range correlations

MB - Moving breather

MLE - Maximum likelihood estimation

OL - Optical lattice

UCF - Universal conductance fluctuations 


\section{Lebenslauf}

Dipl. Phys. Holger Hennig geboren am 29. 10.1976 in Hamburg

Staatsangehörigkeit: deutsch

\begin{tabular}{|l|l|}
\hline $09 / 1983-06 / 1996$ & Schulbesuch in Isernhagen; Abitur \\
\hline $09 / 1996-10 / 1997$ & Zivildienst (Studentenwerk Göttingen) \\
\hline $10 / 1997-07 / 1999$ & Studium der Physik, Universität Göttingen \\
\hline Juli 1999 & Vordiplom in Physik \\
\hline $08 / 1999-03 / 2000$ & Reisen in Australien, Indonesien und Thailand \\
\hline $04 / 2000-03 / 2001$ & Studium der Physik, Freie Universität Berlin \\
\hline $04 / 2001-04 / 2004$ & Studium der Physik, Universität Heidelberg \\
\hline April 2004 & $\begin{array}{l}\text { Diplom in Physik. } \\
\text { Titel der theoretischen Diplomarbeit: } \\
\text { "Ladungswanderung durch Elektronenkorrelation in } \\
\text { Molekülen“. } \\
\text { Ausgeführt am Physikalisch-Chemischen Institut unter } \\
\text { Betreuung von Herrn Prof. Lorenz S. Cederbaum }\end{array}$ \\
\hline $04 / 2004-10 / 2004$ & $\begin{array}{l}\text { Wissenschaftlicher Mitarbeiter, Theoretische Chemie, } \\
\text { Physikalisch-Chemisches Institut, Uni Heidelberg }\end{array}$ \\
\hline seit 11/2004 & $\begin{array}{l}\text { Doktorand am Max-Planck-Institut für Dynamik und } \\
\text { Selbstorganisation, Göttingen und } \\
\text { Institut für Nichtlineare Dynamik, Universität Göttingen }\end{array}$ \\
\hline $\begin{array}{l}\text { Forschungsaufenthalt an der Boston University, } \\
\text { Boston, Massachusetts, USA }\end{array}$ \\
\hline
\end{tabular}

